\title{
CENSUS OF NEW SOUTH WALES MOSSES
}

\author{
HELEN P. Ramsay* \\ (Accepted for publication 29.3.1983)
}

\begin{abstract}
Ramsay, Helen P. (School of Botany, University of New South Wales, Kensington, N.S.W Australia 2033) 1984. Census of New South Wales Mosses. Telopea 2(5): 455-533 - The Census gives a checklist containing 527 species of mosses in 171 genera and 56 families. Compilation of the list is based on literature and information obtained from herbarium specimens in Australian herbaria. The distribution of species is recorded for 13 ecogeographic regions of New South Wales. Included are lists of synonyms, nomina nuda, unsubstantiated records and a bibliography.
\end{abstract}

\section{CONTENTS}

\begin{tabular}{|c|c|c|c|c|c|c|c|c|c|c|c|c|c|c|c|c|c|c|c|}
\hline laterial & & & & & .. & & $\cdot$. & & .. & & .. & . & .. & & & & & & 455 \\
\hline nsus & . & & • & & $\cdot \cdot$ & & • & & $\cdot$ & • & .. & 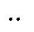 & • & $\ddot{*}$ & .. & : & .. & & \\
\hline nyms & & & .. & * & .. & & . & 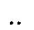 & .. & • & .. & .. & .. & .. & .. & .. & . & .. & $\pi$ \\
\hline tantiated & d reco & & & • & .. & $\cdot$ & .. & .. & .. & .. & .. & .. & .. & .. & .. & .. & .. & $\cdot$ & \\
\hline Von & .. $\quad .$. & .. & .. & .. & .. & .. & .. & .. & .. & .. & .. & .. & .. & .. & .. & .. &.. & .. & \\
\hline 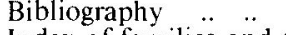 &..$\quad$. & . & .. & .. & .. & .. & .. & .. & .. & .. & .. & .. & .. & .. & .. & .. & .. & .. & \\
\hline ndex of families and & genera & & .. & $\cdot \cdot$ &.. & $\cdot \cdot$ &.. & .. &.. & .. &.. & .. &.. & .. & .. & .. & .. & .. & 532 \\
\hline
\end{tabular}

\section{INTRODUCTION}

The compilation of a checklist of New South Wales mosses and their distribution was undertaken in an effort to determine how much information was available on New South Wales mosses in the literature and in collections based in Australian herbaria. Reverend W.W. Watts and T. Whitelegge early this century (1902, 1905) published lists of Australian mosses, incorporating information from previous work of Mitten $(1856,1860,1882)$ and Forsyth (1899). Watts was responsible for collecting many new species determined by Brotherus in the period 1896-1918. The Census Muscorum Australiensium (Watts and Whitelegge 1902,1905 ) was never completed although manuscripts were in the process of being compiled at the time of Watts' death in 1920; the published lists having dealt only with acrocarpous families.

Further work was continued by Burges $(1932,1935)$ who prepared but never published a census of New South Wales mosses (1952) which incorporated Watts' manuscript and lists for pleurocarpous families (some 526 species in all).

In 1952 Steere remarked that "Since the Census of New South Wales mosses published by Watts and Whitelegge (1902-1905) no further general work (on Australian mosses) has appeared". It is with the kind permission of Professor Burges, and as a former student of his, that $I$ have much pleasure in being able to complete the project to which so many have contributed. There has been an increase in knowledge of Australian mosses during the last 30 years with Sainsbury's New Zealand Mosses (1955a), Scott and Stone's (1976) Mosses of Southern Australia and Catcheside's (1980) Mosses of South Australia, but details of species distribution and of the sub-tropical species in Australia are still very limited. New South Wales has the most interesting and diverse moss flora in Australia since sub-alpine, arid zone, temperate and tropical species representative of a large percentage of the families of mosses all occur in this region. From an

*Also Honorary Research Associate, National Herbarium of New South Wales. 
estimated 1,100-1,200 taxa in Australia (D.G. Catcheside, pers. comm.), New South Wales has about 44\% represented, Queensland about 33\%, Victoria and Tasmania both $30 \%$, South Australia $16 \%$ and Western Australia only $8 \%$. It is hoped the Census will act as a stimulus for future collecting and taxonomic work.

\section{METHODS}

Collections of New South Wales mosses were examined in most Australian Herbaria (AD, BRI, CANB, CBG, HO, NE, NSW, MEL, MELU, SYD, UNSW, UWA) and names and distribution details were obtained for each specimen. The National Herbarium of New South Wales, containing collections of Whitelegge, Forsyth, Watts and others, has been the primary and major source of data for the Census. The distributional data follow the geographical subdivisions used for 'Plants of New South Wales' (Jacobs \& Pickard 1981), see map on p.536; within New South Wales these subdivisions are derived from Anderson (1961). As stated in Jacobs \& Pickard the "Census serves to indicate the state of the art", and this is true for the mosses where our knowledge is in its infancy. Distributional data, for instance, tell us about where people have collected rather than indicating the true range of the species. Since there has been no bryologist engaged on taxonomic work specifically related to mosses in the National Herbarium of New South Wales since W.W. Watts (1916) (see Ramsay \& Briggs 1979) and A. Burges (1952), much is awaiting attention and there are still many unnamed collections.

\section{CRITERIA USED AND ORGANISATION OF DATA}

The Census is arranged by families according to Crosby \& Magill (1978). Within each family, genera, then species are listed alphabetically. Synonyms appear in the Census and are also listed in a separate index. Taxonomic and nomenclatural changes from literature were included up to the time of going to press, data having been derived from Index Muscorum (Wijk et al. 1959-1969) and supplements from Crosby $(1977,1979$, Crosby \& Bauer 1981) as well as from published revisions by individual taxonomists (see Bibliography). Name changes in literature have been accepted as relating to the name on the packet unless, or until, the specimen has been examined by an expert or renamed.

It must be stressed that verification has only been possible where specialists have borrowed and checked determinations. The work contains only published names and combinations except in the genus Macromitrium for which a revision of Australian species is in manuscript (Vitt \& Ramsay) and Racopilum under review by van Zanten who has examined all relevant specimens.

A taxon is recorded as present in any region if at least one specimen with that name is available in an Australian herbarium or private collection. Reliability of identification may be questioned where few specimens of a particular taxon have been found and for this reason where collections are for 3 or fewer specimens for a particular area this is indicated. Species records not supported by specimens are listed separately at the end of each genus as 'previous record not substantiated'. Nomina nuda which have not been accounted for in the synonymy in the Census are treated separately (p.509).

Distribution of taxa in other states of Australia (extra-N.S.W.) is indicated as well as the extra-Australian range. These data are included for convenience but are not necessarily definitive. Within New South Wales, distribution is shown by the ecogeographic regions used by Jacobs \& Pickard (1981) based on Anderson (1961). Lord Howe Island species are included since the island is politically part of New South Wales. The distribution also encompasses the Australian Capital Territory (A.C.T.) which is geographically located in the Southern Tablelands Division of New South Wales. Extra-Australian distributions follow the regions named in Jacobs \& Pickard (1981) and listed on page 459 here. 
The paucity of species and collections within some regions highlights the need for further work and more intensive surveys of distribution. The original data, which include collectors' notes for some 20,000 specimens, will remain available so that lists within regions may be compiled and added to as new collections are made, particularly with a view to increasing our knowledge of ecological preferences for species. Already it is clear that many species have been collected from only a limited area or have a very restricted distribution. Such species should be studied thoroughly and efforts made to determine their true range.

This Census recognises 527 species (not including 22 unsubstantiated records) in 171 genera from 56 families of mosses. A number of infraspecific taxa add to the total species diversity in the area. In all, 81 species are listed as being restricted in distribution to New South Wales (Ramsay 1984a). Although a large number of new records are included e.g. Drepanocladus uncinatus at Mt. Kosciusko, Brachymenium exile, these have not been highlighted in the list. Relationships between New South Wales species and those in other states of Australia indicate a separation into those sub-tropical species that have been recorded from New South Wales and Queensland only, and those (the majority) with affinities to the temperate bryoflora of southern Australia. Of the sub-tropical species many have been collected only in the MacPherson-Macleay overlap on the border between New South Wales and Queensland. This region is considered as phytogeographically significant for higher plants (Burbidge 1960). A preliminary survey of phytogeographical relationships of the mosses of New South Wales is included separately (Ramsay 1984a). The distributions of mosses within the Australian Capital Territory (Ramsay \& Streimann 1984) and on Lord Howe Island (Ramsay 1984b) have also been analysed in separate papers (see pp. 559, 549).

\section{ACKNOWLEDGEMENTS}

Financial support from the Australian Biological Resources Study from 1974 to 1979 (incl.) to enable distributional information to be recorded necessitated the complete sorting and repackaging of specimens into a uniform system at the National Herbarium of New South Wales. Without the co-operation of the Director and Staff at the Herbarium in allowing me access at all times and their contribution in repackaging and labelling the collections, the task could not have been completed.

Many others have also assisted freely. The Directors and Staff of various Herbaria (AD, BRI, CANB, CBG, HO, NE, NSW, MEL, MELU, SYD, UNSW, UWA) have helped greatly by making collections and facilities available and supplying information when requested. Dr A. Touw, Rijksherbarium, Leiden, has contributed much time and effort in identifying Streimann's collections from the National Botanic Gardens. Help has also been given by Staff of the British Museum and the V.F. Brotherus Herbarium at Helsinki. Personal thanks to M. Austin, B. Austin, D.G. Catcheside, G. Harden, T. Howard, A. Murray, J. Parram, L. Pedley, G.A.M. Scott, I.G. Stone, H. Streimann and J.H. Willis, who have given me specimens, supplied unpublished lists and/or other information, checked sections of the manuscript, or allowed me access to their private collections. My two assistants, T. Boyd (1976) and P. Wilson (1978), gave invaluable help in sorting, checking and recording data and in collecting and assembling the data for the manuscript. C. Puttock helped with checking, indexing and literature lists. Assistance in reading sections of the manuscript and helpful suggestions given by H. Ando, D.G. Catcheside, T. Koponen, W. Margadant, R. Pursell, G.A.M. Scott, I.G. Stone, D.H. Vitt and B.O. van Zanten are also gratefully acknowledged. Extra distributional data supplied by I.G. Stone were obtained during travel for research projects funded by the Australian Research Grants Committee. The following people examined and checked identification of genera and species of special interest to them - H. Ando, A. Touw, B.O. van Zanten, I.G. Stone, M.A. BruggemannNannenga, J. Lewinsky, D.H. Vitt, T. Koponen, R.D. Seppelt and H. Mohamed. 
Their expertise and co-operation proved a most valuable contribution. A. Lonergan, S. Wray, J. Ratcliffe and F. Colman have helped enormously by typing drafts and the final manuscript. The encouragement and patient support of my husband has been of immense help in enabling the work to be completed.

The author accepts full responsibility for any errors or omissions and would appreciate notification of any inaccuracies.

\section{ADDENDA}

One species of Archidium Brid. (Archidiaceae) has now been found in New South Wales (I.G. Stone, pers. comm.).

I.G. Stone (pers. comm.) reports the following new records for New South Wales. Details to be published later.

Bruchia eckloniana C. Muell. - NWS; V; S Afr

Ephemerum rehmannii (C. Muell.) Broth. - SFWP; VSW

Tortula rhizophylla (Sak.) Iwats. \& Saito - CC; Q; S Afr

The following references have been added since the manuscript was submitted for publication.

Althofer, G.W. \& Harden, G.J. (1980). 'The Flora of Mt. Arthur Reserve (Wellington, N.S.W.).' (Trustees of Mt. Arthur Reserve: Wellington.)

Crosby, M.R. \& Bauer, C.R. (1983). Index Muscorum Supplementum - 1980-1981. Taxon 32(4): 670-691.

Fife, A.J. (1982). Taxonomic and nomenclatural observations on the Funariaceae 1. Physcomitrium, Physcomitrella and Goniomitrium in New Zealand. Lindbergia 8: 96-104.

Frahm, J.P. (1982). Gross disjunktionen von Arealen sudamerikanischer und Afrikanischer Campylopus-arten. Lindbergia 8: 45-52.

Geissler, P. \& Green, S.W. (1982). Bryophyte taxonomy. Nova Hedwigia 71: 1-558.

Harris, S. \& Ratkowsky, D.A. (1982). A new moss record from Tasmania, Ischyrrhodon lepturus (Tayl.) Schelpe. Tasmanian Naturalist 71: 7.

Koponen, A. (1982). The family Splachnaceae in Australasia and the Pacific. J. Hattori Bot. Lab. 52: 87-92.

Lewinsky, J. (1984). The genus Orthotrichum Hedw. (Musci) in Australasia: a taxonomic revision. J. Hattori Bot. Lab. 56 (in press).

Ochi, H. (1980). A revision of the neotropical Bryoideae, Musci (first part). J. Fac. Educ. Tottori Univ. Nat. Sc. 29: 49-154.

Ramsay, H.P. (1983a). A moss with four chromosomes. Lindbergia 9(2): 89-92.

Ramsay, H.P. (1983b). Cytology of mosses. In Schuster, R.M. (ed.), 'New Manual of Bryology.' (Hattori Botanical Laboratory: Nichinan.) Vol. 1, pp.149-221.

Ramsay, H.P. \& Vitt, D.H. (1984). Cytotaxonomy, distribution and sexuality of Macromitrium in the Pacific. J. Hattori Bot. Lab. 55: 23-33.

Schuster, R.M. (1983). 'New Manual of Bryology.' (Hattori Botanical Laboratory: Nichinan.) 2 vols.

Scott, G.A.M. (1982a). Bryofloristics in Australasia. Nova Hedwigia 71: 483-494.

Scott, G.A.M. (1982b). Desert bryophytes. In Smith, A.J.E. (ed.) 'Bryophyte Ecology.' (Chapman and Hall: London, New York.)

Seppelt, R.D. (1980a). A synoptic moss flora of Macquarie Island. Antarctic Division Technical Memorandum 93: 1-8.

Seppelt, R.D. (1980b). A synoptic moss flora of Macquarie Island II. Sphagnum. Antarctic Division Technical Memorandum 98: 1-9. 
Seppelt, R.D. (1981a). A synoptic moss flora of Macquarie Island III. Fissidens. Antarctic Division Technical Memorandum 100: 1-9.

Seppelt, R.D. (1981b). Studies on the bryoflora of Macquarie Island III. Collections, new moss additions and corrections, and a revised checklist. Bryologist 84(2): 249-252.

Seppelt, R.D. (1982). An overview of the Ditrichaceae. Nova Hedwigia 71: 215-218.

Stone, I.G. (1982). Some new and noteworthy records of mosses mostly from Queensland, Australia. Austrobaileva (1)5: 511-520.

\section{ABBREVIATIONS AND SYMBOLS USED IN TABLE 1}

\section{Distribution within New South Wales}

L.HI Lord Howe Island

$\mathrm{C}$ Coast

T Tablelands

WS Western Slopes

WP Western Plains
FWP Far Western Plains

N North

C Central

S South

NSW New South Wales

- present in the subdivision $>3$ collections

o no sighted or cited specimen from the subdivision

? specimen or cited locality inadequate for reliable record; in the extra-NSW column of Table 1 the '?' precedes the abbreviation for the state to which the doubtful record applies.

$\star$ number of specimens $\leqslant 3$

\section{Extra-New South Wales Distribution}

Q - Queensland, V - Victoria, T - Tasmania, Y - Northern Territory, S South Australia, W - Western Australia, A - Australian Capital Territory (included separately here although it lies within the geographical boundaries of N.S.W. [see Ramsay \& Streimann 1984] as it is a political entity)

\section{Extra-Australian Distribution}

\begin{tabular}{|c|c|c|c|}
\hline $\mathrm{N}$ & $\begin{array}{l}\text { North, northern } \\
\text { or New }\end{array}$ & Malesia & $\begin{array}{l}\text { The Malay Peninsula, } \\
\text { Philippines, Malaysia, }\end{array}$ \\
\hline $\mathrm{S}$ & South, southern & & Indonesia and New \\
\hline $\mathrm{E}$ & East, eastern & & Guinea, a phytogeographic \\
\hline W & West, western & & not a political region \\
\hline $\mathrm{C}$ & Central & Medit & Mediterranean region \\
\hline cosmop & cosmopolitan & N Caled & New Caledonia \\
\hline Afr & Africa & $\mathrm{NG}$ & New Guinea \\
\hline Amer & America & $\mathrm{NZ}$ & New Zealand \\
\hline As & Asia & Subant I & Subantarctic Islands \\
\hline Eur & Eur & Oc & Oceania \\
\hline I & Island or Islands & $\mathrm{Pac}$ & Pacific \\
\hline & & Philip & Philippines \\
\hline
\end{tabular}


TABLE 1. DISTRIBUTION AND CHECKLIST OF MOSSES OF NEW SOUTH WALES.

(Families arranged according to Crosby \& Magill 1981.) For index to genera and families see p. 532.

O absent

- $>3$ collections

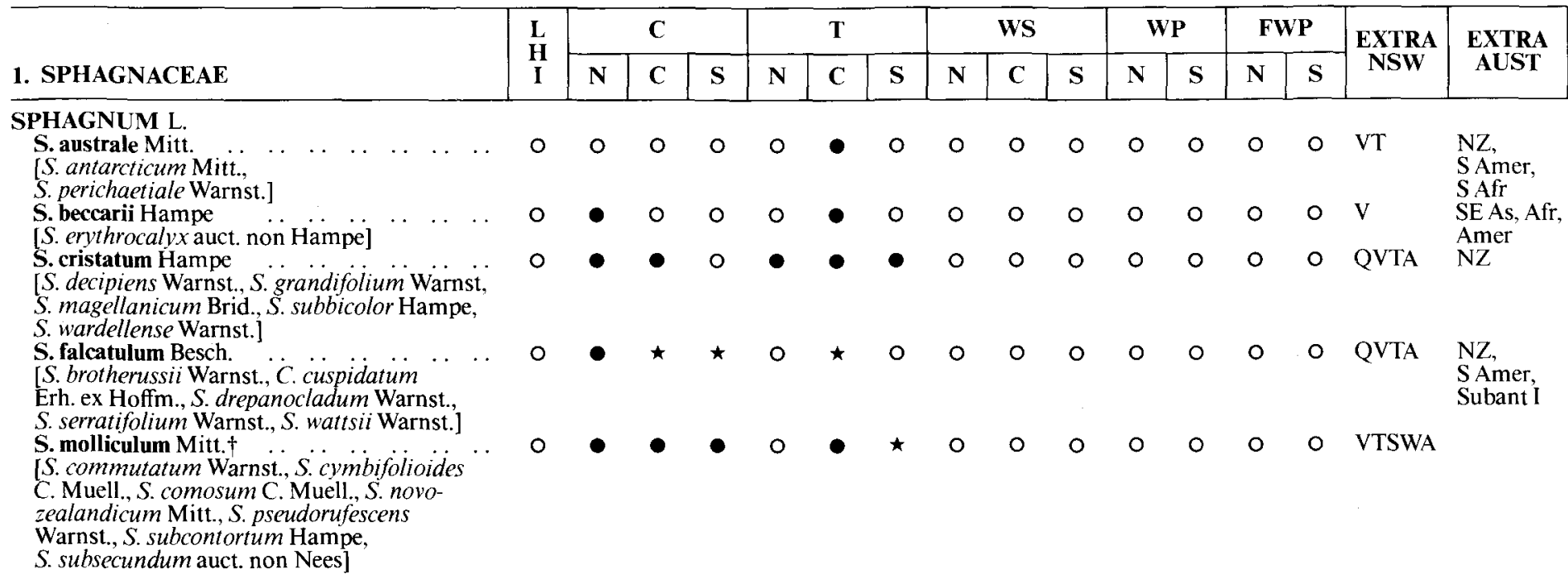

\section{ANDREAEACEAE}

\section{ANDREAEA Hedw.}

A. australis Mitt. ...

A. ambitis Hook. f. \& Wils.

A. amblyophylla C. Muell. ex Broth.

A. rupestris auct. non Hedw.

A. nitida Hook f. \& Wils. 
A. subulata Har. ex Hook

[A. subulatissima C. Muell.]

000

A. tenera C. Muell.

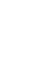

0000

\section{FISSIDENTACEAE}

FISSIDENS Hedw.

F. amblyothallioides Broth. \& Watts .. ..

F. anisophyllus Dix.

F. arboreus Broth

F arcuatulus Broth \& $\begin{array}{llllll}\text { Watts } & \cdots & \cdots & \cdots & .\end{array}$

F. aristatus Broth

F asplenioides Hedw.

F australiensis Jaeg.

$\begin{array}{lllllll}0 & 0 & \star & 0 & 0 & 0 & 0 \\ 0 & 0 & 0 & 0 & \star & 0 & 0 \\ 0 & 0 & \star & 0 & 0 & 0 & 0 \\ 0 & 0 & \star & 0 & 0 & 0 & 0 \\ 0 & 0 & \star & 0 & 0 & 0 & 0 \\ 0 & \star & \star & \star & 0 & \star & \star\end{array}$

F. basilaris C. Muell. \& Hampe

F. bryoidioides Broth

F. calodictyon Broth.

F. cambewarrae Dix

F. crassipes Wils. ex B.S.G.

$[F$. dietrichiae $\mathrm{C}$. Muell.

$F$. praemollis Broth

F. dealbatus Hook. f. \& Wils. . . . . . . ..

F. fontanus (Pyl.) Steud.

[F. muelleri (Hampe) Mitt.]

F forsythii Broth.

. forsythii Broth

F. homomallulus C. Muell. ex Dix.

F. humilis Dix. \& Watts

F. leptocladus $C$. Muell. ex Rodway

F. longiligulatus Broth. \& Watts

F. montecollii Broth. \& Watts

F. montecollibrolh. \& Watts

$\begin{array}{lll} & \cdots & \cdots \\ & \cdots & \cdots \\ \cdots & \cdots & \cdots \\ \cdots & \cdots & \cdots \\ & \cdots & \cdots\end{array}$

$\begin{array}{lll}\cdots & \cdots & \cdots \\ \cdots & \cdots & \cdots\end{array}$

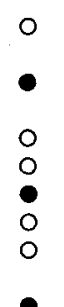

0 $\begin{array}{lllllll}0 & \star & 0 & 0 & 0 & \star & 0 \\ 0 & 0 & 0 & 0 & 0 & 0 & 0 \\ 0 & 0 & 0 & 0 & 0 & 0 & 0 \\ 0 & 0 & 0 & \star & 0 & \star & \star \\ 0 & \star & \star & \star & \star & \star & 0\end{array}$

0000

- 0
NZ

QVTYS- Cosmop

WA (excl. Oc)

S

$Q$
Eur, As,
Afr,
VT NA, Fiji,
OC
QVTS Cosmop

$\begin{array}{ll}\text { QVYA } & \text { NZ } \\ \text { QVTS } & \text { NZ }\end{array}$

WA

$\stackrel{5}{5}$ 


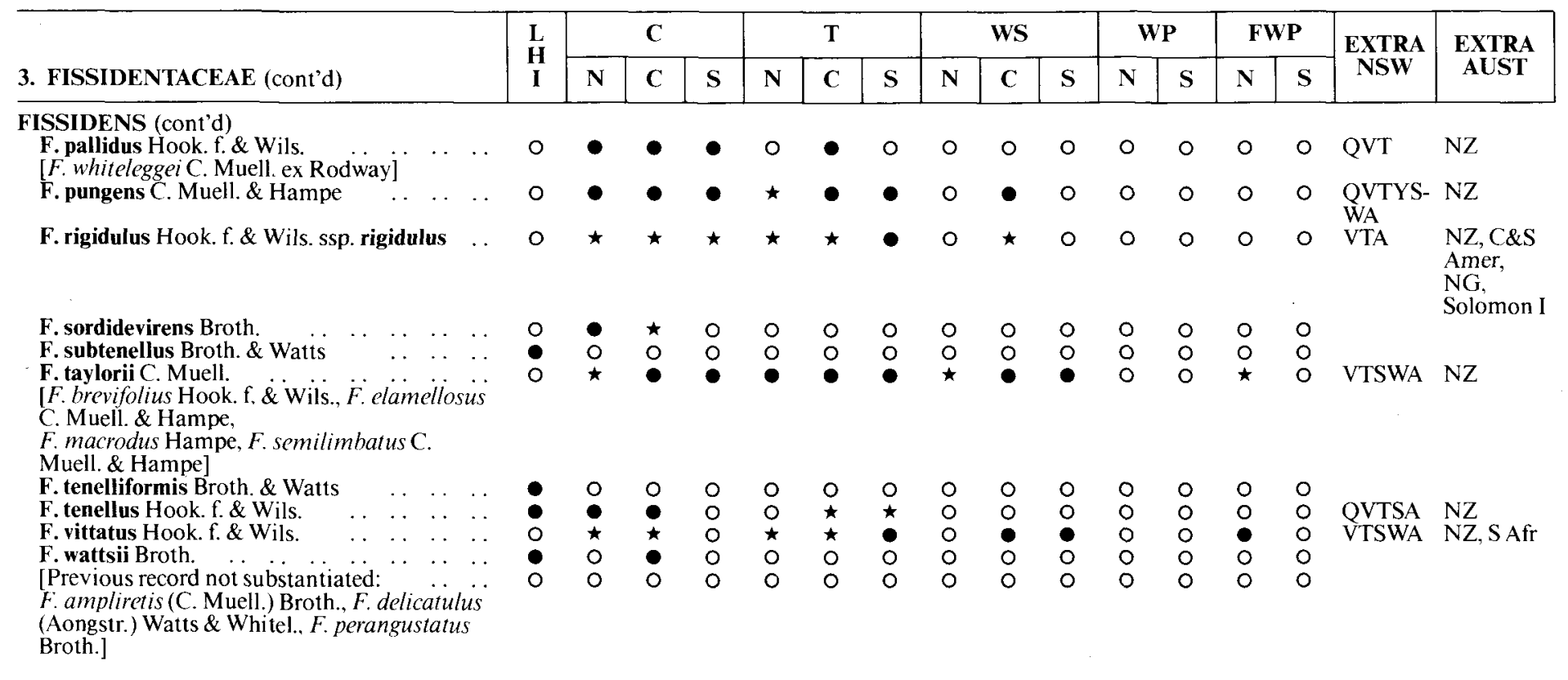

\section{DITRICHACEAE}

CHRYSOBLASTELLA Williams

C. chilensis (Mont) Reim.

[Cheilothela chilensis (Mont.) Broth.]

\section{CERATODON Brid.}

C. purpureus (Hedw.) Brid.

[C. convolutus Reichdt.

C. stenocarpus Bruch. \& Schimp. ex C. Muell.]

DISTICHIUM B.S.G.

D. capillaceum (Hedw.) B.S.G.

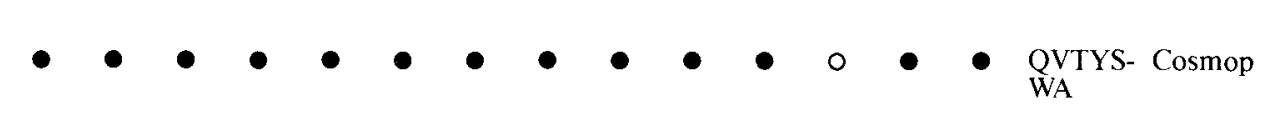




\section{DITRICHUM Hampe}

D. brachycarpume

D. brevirostre (R. Br. ter.) Broth

[D. subbrachycarpum (C. Muell.) Par.]

D. cylindricarpum (C. Muell.) F. Muel

[D. elongatum (Hook. f. \& Wils.) Mitt.

D. strictum (Hook. f. \& Wils.) Hampe]

D. difficile (Dub.) Fleisch.

[D. affine (C. Muell.) Hampe, D. bailevi

C. Muell. nom. mud., D. flaccidulum C. Muell.

in Watts \& Whitel.. D. flexifolium Hampe

D. muelleri (Hampe) Hampe, D. setosum

(Hook. f. \& Wils.) Reichdt.]

D. punctulatum Mitt.

D. rufo-aureum (Hampe) Willis

[Aongstroemia rufo-aurea Hampe]

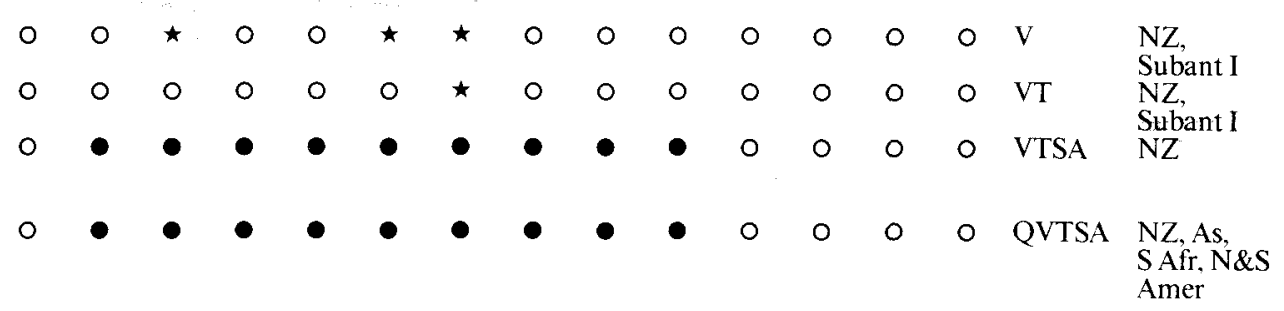

CCREMIDIUM Wils.

$\begin{array}{lllllllllllllllllll}\text { E. arcuatum (Hook. f. \& Wils.) C. Muell. } & \ldots & 0 & 0 & 0 & 0 & 0 & 0 & 0 & \star & 0 & 0 & 0 & \star & 0 & 0 & Q V S W \\ \text { E. brisbanicum (Broth.) Stone \& Scott } & \cdots & 0 & \bullet & \bullet & \bullet & 0 & \star & 0 & 0 & 0 & 0 & 0 & 0 & 0 & 0 & Q\end{array}$

Micromitrium brisbanicum (Broth.) Crosby,

Nanomitrium brisbanicum (Broth.) Broth.,

Pleuridium austro-subulatum Broth. ex Roth.]

E. exiguum (Hook. f. \& Wils.) Wils.

E. minutum (Mitt.) Stone \& Scott

[Ephemerum whiteleggei Broth. \& Geh.,

Bruchia whiteleggei C. Muell.,

Sporledera whiteleggei (C. Muell.) Broth.]

E. pulchellum (Hook. f. \& Wils.) C. Muell.

[Archidium stolonaceum C. Muell.]

E. whiteleggei Broth.

[Sporledera minutissima (Dix. \& Sainsb.)

Sainsb.]

PLEURIDIUM Rabenh.

P. arnoldii (R. Br, ter.) Par. ${ }_{\text {P. }}$. $\quad \ldots \quad \ldots \quad \ldots$

$\begin{array}{lllllll}\text { P. brachycaulon (C. Muell.) Kindb. } & \ldots & \ldots \\ \text { P. curvisetum Stone } & \ldots & \ldots & \ldots & \ldots & \ldots & \ldots\end{array}$

P. krauseanum Par.

$\begin{array}{ll}0 & 0 \\ 0 & 0 \\ 0 & 0 \\ 0 & 0\end{array}$

$\begin{array}{ll}0 & 0 \\ 0 & 0 \\ 0 & 0 \\ 0 & 0\end{array}$

0
0
0
0

0
0
0

$\begin{array}{lll}\star & 0 & 0 \\ 0 & 0 & 0 \\ \star & 0 & 0 \\ \star & 0 & 0\end{array}$

$\begin{array}{llllll}0 & 0 & 0 & 0 & 0 & \text { VA } \\ 0 & 0 & 0 & 0 & 0 & V \\ 0 & 0 & 0 & 0 & 0 & \\ 0 & 0 & 0 & 0 & 0 & V S A\end{array}$

NZ

NZ, S Afr

Subant 1

NZ 


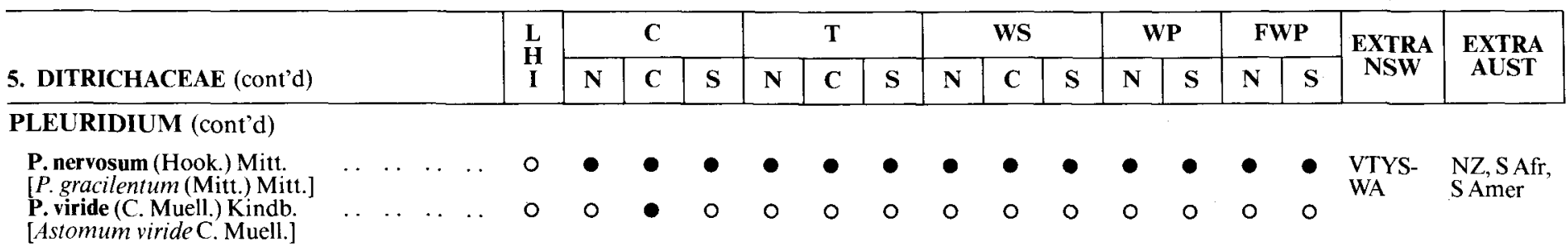

\section{SELIGERIACEAE}

\section{BLINDIA B.S.G}

B. magellanica Schimp.

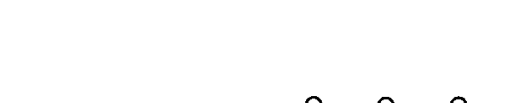

\section{DICRANACEAE}

\section{BRUCHIA Schwaegr.}

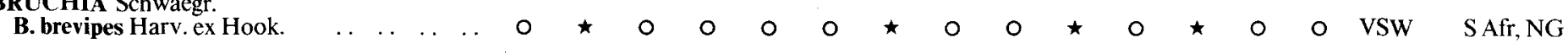

CAMPYLOPUS Brid.

C. austro-subulatus Broth. \& Geh. $\dagger \quad \ldots \quad \ldots$

C. bicolor (C. Muell.) Wils.

C. clavatus (R. Brown) Wils.

[C. appressifolius Mitt

C. institius Hook. f. \& Wils.]

C. denticuspis Broth. var. lutescens Broth.

C. introflexus (Hedw.) Brid

[C. pudicus (C. Muell.) Jaeg.

C. tasmanicus Par.]

C. pallidus Hook. f. \& Wils.

[C. novae-valesiae Broth, C. torquatus Mitt.,

?C. woollsii (C. Muell.) Par.]

C. perauriculatus Broth.

C. perauriculatus Broth.
C. viridicatus (C. Muell.) Par.

$\circ$

$\begin{array}{llllllllllllllll}\circ & 0 & 0 & 0 & 0 & 0 & \star & 0 & 0 & 0 & 0 & 0 & 0 & 0 & \text { QVSA } \\ 0 & \star & \bullet & \star & 0 & \bullet & \star & 0 & 0 & \star & 0 & 0 & 0 & 0 & \text { QVTSW NZ, Afr } \\ \bullet & \bullet & \bullet & \star & \star & \bullet & \bullet & \star & \bullet & \star & 0 & 0 & 0 & 0 & \begin{array}{l}\text { QVVTS- NZ, Afr, } \\ \text { WA }\end{array}\end{array}$

. $\quad . . . \quad$.

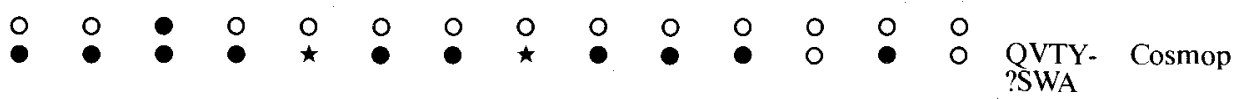
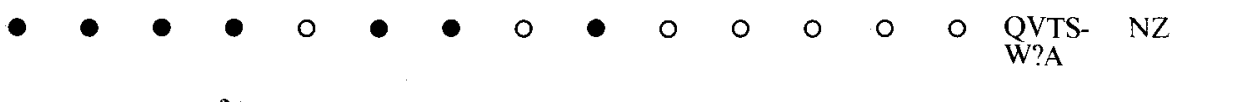

$\begin{array}{lllllllllllllll}0 & 0 & 0 & ? \star & 0 & 0 & 0 & 0 & 0 & 0 & 0 & 0 & 0 & 0 & \\ 0 & 0 & \star & 0 & 0 & 0 & 0 & 0 & 0 & 0 & 0 & 0 & 0 & 0 & Q\end{array}$

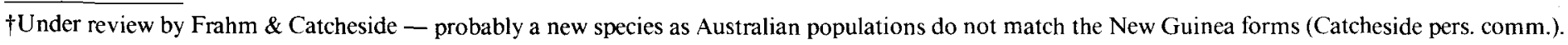


DICNEMOLOMA (Ren.) Ren. $\ddagger$

D. pallidum (Hook.) Wijk \& Marg.

[D. sieberianum (Hornsch.) Broth.

Leucoloma sieberianum (Hornsch.) Jaeg.

Sclerodontium pallidum Hook.]

[Previous record not substantiated:

D. fraseri (Mitt.) Ren., D. imbricatum Broth.

\& Geh., D. strictipilum (C. Muell.) Par.

DICRANELLA (C. Muell.) Schimp.

D. apophysatula (C. Muell.) Broth.

D. cardotii (R. Br. ter.) Dix.

[D. wattsii Broth.,

Dichodontium wattsil (Broth.) Broth.]

D. dietrichiae (C. Muell.) Jaeg. $\quad \ldots \quad \ldots \quad \ldots \quad$ o

[D. tricruris (C. Muell) Mitt]

D. jamesonii (Mitt) Broth.

Anisothecium jamesonii Mitt.

D. pycnoglossa (Broth.) Kindb.

D. pycnoglossa (Broth.) Kindb.

[Anisothecium pvcnoglossum Broth.]

[Previous record not substantiated:

Aongstroemia stackhousiana C. Muell.]

DICRANOLOMA Ren.

D. argutum (Hampe) Par.

[Dicranum whiteleggei C. Muell.]

D. austro-scoparium (Broth.) Watts \& Whitel.

[Leucoloma austro-scoparium C. Muell. ex

Broth.]

D. billardieri (Brid.) Par.

D. dicarpum Nees Par.

[Dicranum dicarpum (Nees)

Leucoloma dicarpum (Nees) Broth.]

D. menziesii (Tayl.) Par.

D. robustum (Hook. f. \& Wils.) Par, var

setosum (Hook. f. \& Wils.) Sainsb.

D. serratum (Broth) Par.

Leucoloma serratum Broth.]

Deucoloma serratum Broth.]

[Dicranum sullivanil C. Muell.]

†Retained here as Dicnemoloma although Crosby (pers. comm.) and Crosby \& Magill (1981) point out this name is preceded by Sclerodontium. 


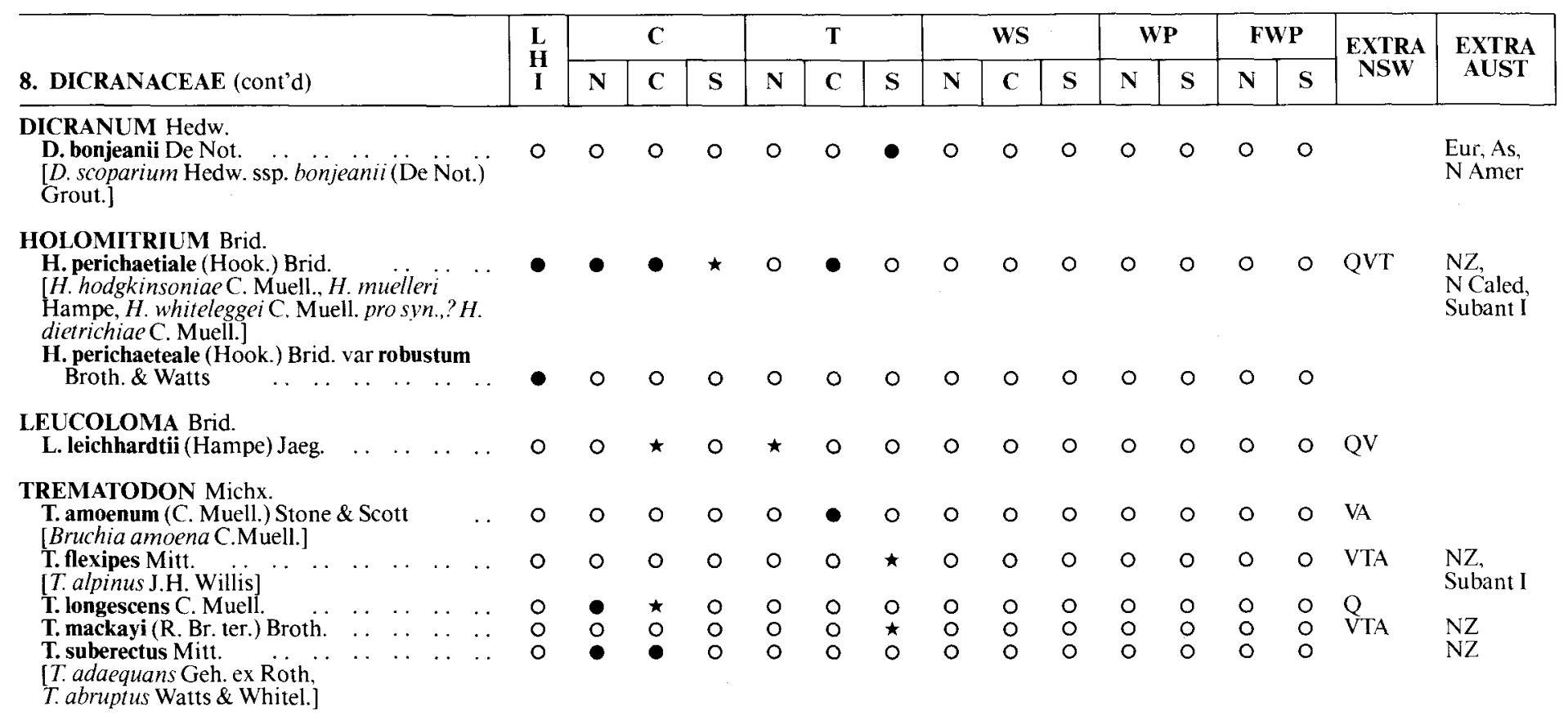

\section{DICNEMONACEAE}

EUCAMPTODON (Mont.) Mont.

E. muelleri Hampe \& C. Muell.

\section{LEUCOBRYACEAE}


L. candidum (P. Beauv.) Wils.

[L. brachyphyllum Hampe,

L. teysmannianum Dozy \& Molk.,

L. strictifolium Broth.]

L. candidum (P. Beauv.) Wils. var.

pentastichum (Dozy \& Molk.) Dix.

[L. spirostichum C. Muell. in Mitt. nom. nud.

L. speirostichum C. Muell. in Geh. nom. nud.]

L. turgidulum C. Muell.

L. wattsii Broth.

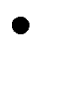

:

12. CALYMPERACEAE

CALYMPEROPSIS (C. Muell.) Fleisch.

C. wattsii (Broth.) Fleisch.

[Syrrhopodon wattsi Broth.]

SYRRHOPODON Schwaegr.

S. fimbriatulus C. Muell.

S. incompletus Schwaegr.

[Calymperes armatum Broth.]

S. novae-valesiae C. Muell.

S. platyceri Mitt.

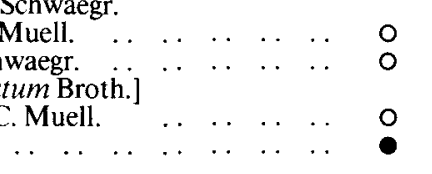

$\circ$

$\circ \quad 0$

0

$\circ 0$

- QVTW

NZ, Oc

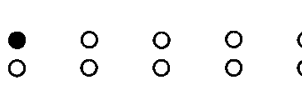

0
0
0

$\stackrel{\circ}{\circ}$

0
0

$\circ \quad 0$

$\mathrm{NZ}$
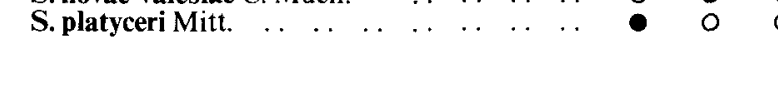

$$
\text { o }
$$

$\begin{array}{lllllll}0 & 0 & 0 & 0 & 0 & 0 & \\ 0 & 0 & 0 & 0 & 0 & 0 & \\ 0 & 0 & 0 & 0 & 0 & 0 & Q\end{array}$

13. ENCALYPTACEAE

\section{ENCALYPTA Hedw.}

E. vulgaris Hedw. $\ldots$. . . . . .

[Previous record nol substantiated:

E. novae-valesiae Hampe] 


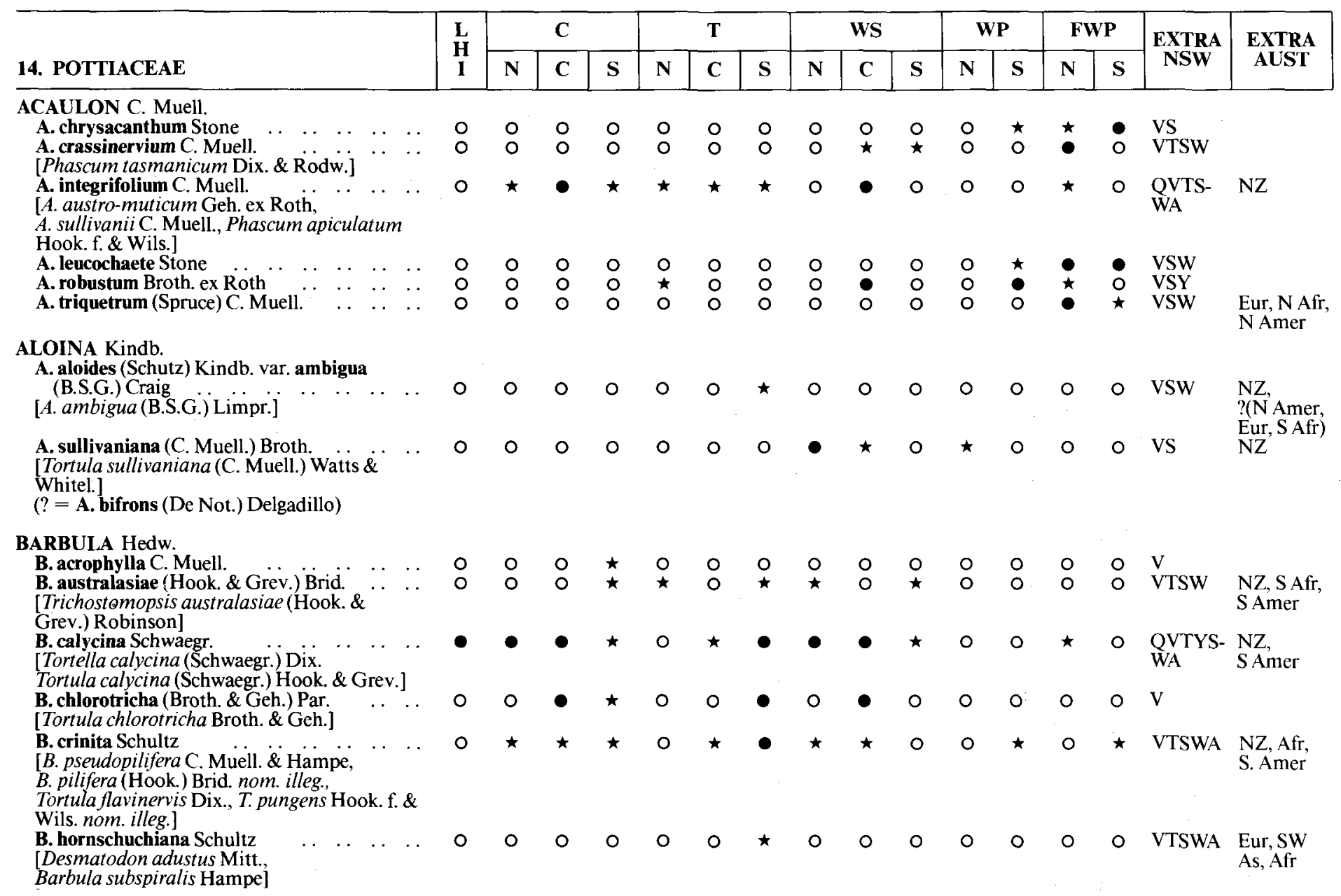


B. speirostega C. Muell

B. subcalycina C. Muel

[Tortula subcalycina (C. Muell.) Mitt.]

B. torquata Tayl.

[Tortula torquata (Tayl.) Wils.]

[Tortula acuminata Brid. nom. illeg.]

\section{BRYOERYTHROPHYLLUM Chen.}

B. rotundatum (Lindb. \& Arn.) Chen.

[Barbula rotundata Lindb. \& Arn.

Didymodon rotundatus (Lindb. \& Arn.) Par.]

B. jamesonii (Tayl.) Crum.

CALYPTOPOGON (Mitt.) Broth.

C. mnioides (Schwaegr.) Broth.
$\begin{array}{llll}0 & 0 & 0 & 0 \\ 0 & 0 & 0\end{array}$
0
$\begin{array}{ll}\star & 0 \\ 0 & 0\end{array}$
0
00
-
○
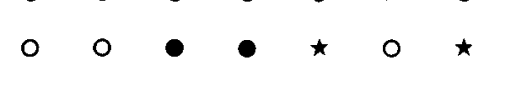
0
$\begin{array}{lllllll}\circ & \circ & \circ & \circ & \circ & \circ & \\ 0 & \circ & \circ & \circ & 0 & \circ & V S W\end{array}$
- V VTYSW- NZ,
- $\quad \stackrel{\text { VSTA }}{\text { CAmer }}$

CROSSIDIUM Jur.

C. davidai Catcheside

C. geheebii (Broth.) Broth

DESMATODON Brid.

D. convolutus (Brid.) Grout.

Tortula atro-virens (Sm.) Lindb

Barbula atro-virens (Sm.) Schimp.

Pottia brachyodus (Hampe) Jaeg.]

D. recurvatus (Hook.) Mitt.

DIDYMODON Hedw.

D. wildii (Broth.) Broth.

GYMNOSTOMUM Nees \& Hornsch.

G. aeruginosum (Sm.) Zander

[G. calcareum Nees \& Hornsch.]

HYMENOSTOMUM R. Br.

H. olivaceum C. Muell. ex Geh.

H. sullivanii C. Muell. ex Geh.

LEPTODONTIUM (C. Muell.) Hampe ex Lindb.

L. viticulosoides ( $\mathrm{P}$. Beauv) Wijk \& Marg

[Trichostomum subdenticulatum C. Muell.]

L. paradoxum Stone \& Scott

[L. interruptum (Mitt.) auct. non Broth.] 


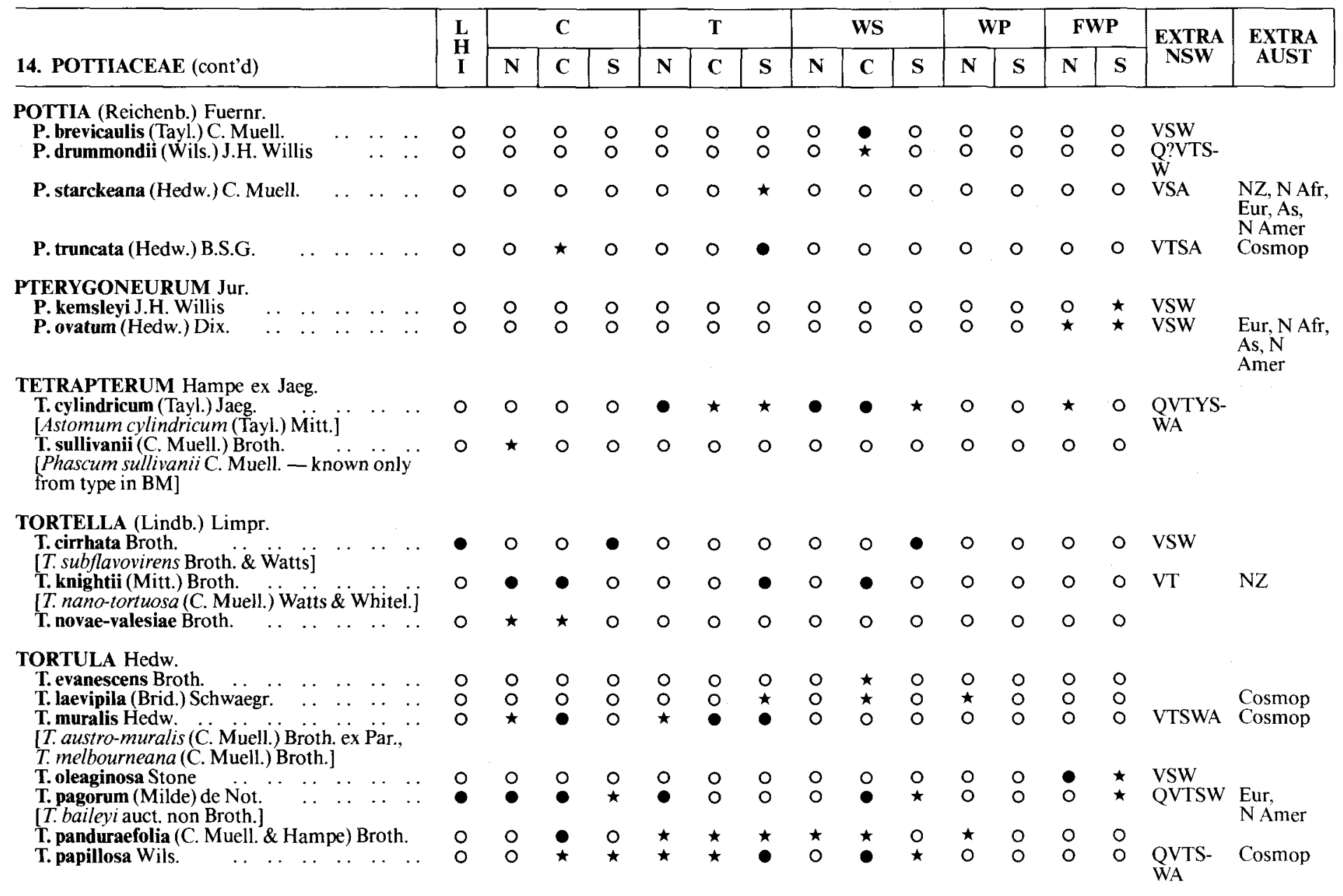




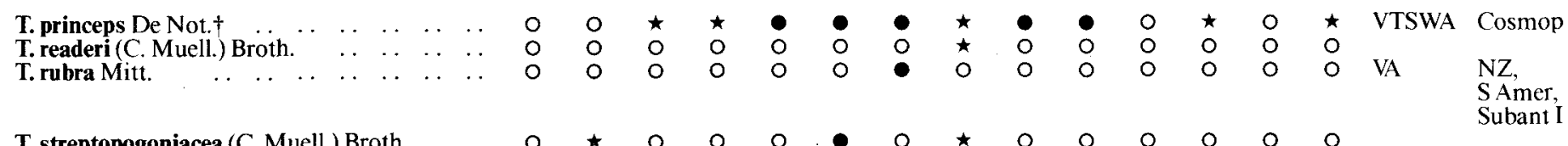

T. streptopogoniacea (C. Muell.) Broth.

T. subbrunnea Broth. \& Watts

T. vesiculosa (C. Muell.) Broth.

[Previous record not substantiated:

T. crawfordii (Par.) Watts in Watts \& Whitel.,

T. asperifolia Broth.,

T. parramattana Mitt.,

Barbula parramattana (Mitt.) Kindb.]

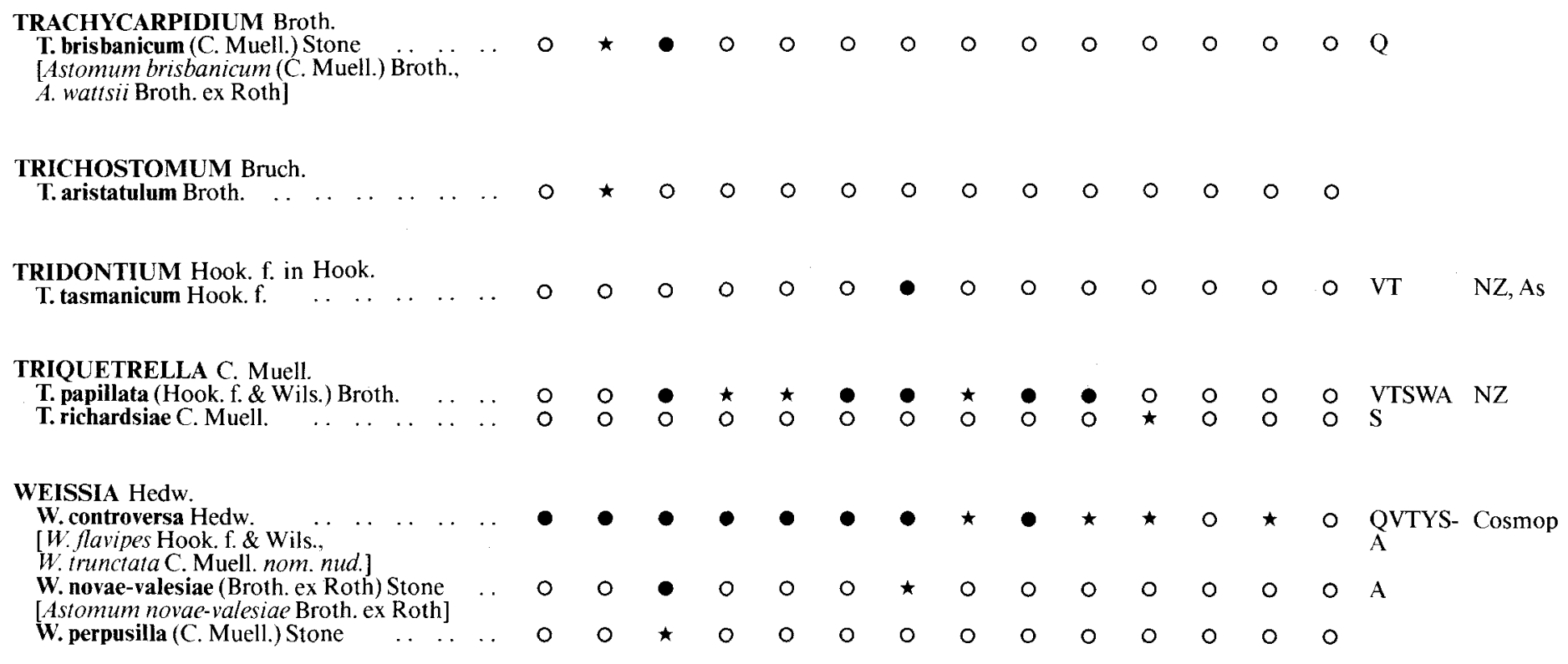

†NSW collections of this species examined by Kramer (pers.comm.) include T. andersonii, T. antarctica, T. mbella, and T. lencostega as well as T. princeps. 


\begin{tabular}{|c|c|c|c|c|c|c|c|c|c|c|c|c|c|c|c|c|}
\hline \multirow[b]{2}{*}{ 14A. BRYOBARTRAMIACEAE } & \multirow{2}{*}{$\begin{array}{c}\mathbf{L} \\
\mathbf{H} \\
\mathbf{I}\end{array}$} & \multicolumn{3}{|c|}{ C } & \multicolumn{3}{|c|}{$\mathbf{T}$} & \multicolumn{3}{|c|}{ WS } & \multicolumn{2}{|c|}{ WP } & \multicolumn{2}{|c|}{ FWP } & \multirow{2}{*}{$\begin{array}{c}\text { EXTRA } \\
\text { NSW }\end{array}$} & \multirow{2}{*}{$\begin{array}{c}\text { EXTRA } \\
\text { AUST }\end{array}$} \\
\hline & & $\mathbf{N}$ & $\mathbf{C}$ & $\mathbf{S}$ & $\mathbf{N}$ & C & $\mathbf{S}$ & $\mathbf{N}$ & C & $\mathbf{S}$ & $\mathbf{N}$ & $\mathbf{S}$ & $\mathbf{N}$ & $\mathbf{S}$ & & \\
\hline
\end{tabular}

\section{BRYOBARTRAMIA Sainsb.}

B. novae-valesiae (Broth.) Stone

[Trachycarpidium novae-valesiae Broth.,

Bryobartramia robbinsii Sainsb.]

\section{GRIMMIACEAE}

\section{GRIMMIA Hedw.}

G. donniana Sm.

[G. alpestris Sommerf.]

G. laevigata (Brid.). Brid.

G.

[G. leiocarpa Tayl., G. leucophaea Grev.

000000000

(1)

10

o 0 O VSWA S Afr

$G$. campestris Burchell ex Hook.]

G. obtusata (C. Muell. \& Hampe Jaeg.
G. pulvinata (Hedw.) Sm. var. africana

(Hedw.) Hook. f. \& Wils.

[G. cygnicolla Tayl., G. pulvinata (Hedw)

var.

v. trichophylla Grev.

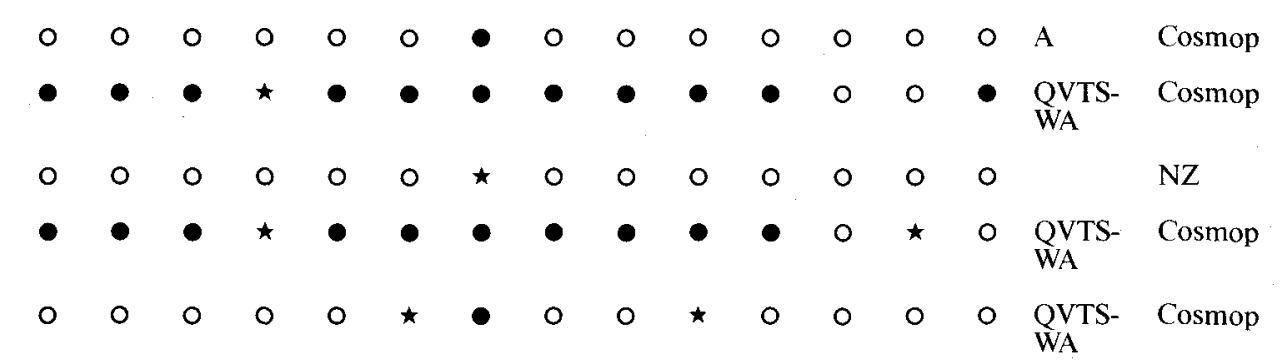

\section{RACOMITRIUM Brid.}

R. aciculum Dix. ‥

R. amoenum (Broth.) Par. $\ldots \quad \ldots \quad \ldots \quad \ldots$

R. crispulum (Hook. f. \& Wils.) Hook. f. \&

[R. sullivanii (C. Muell.) Broth.,

$R$. symphodon Jaeg.]

R. lanuginosum (Hedw.) Brid. var.

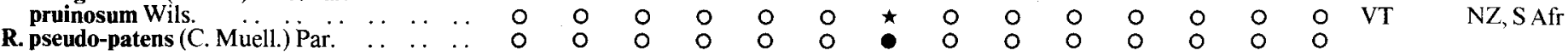

SCHISTIDIUM Brid.

S. apocarpum (Hedw.) B.S.G.

[Grimmia apocarpa Hedw. nom. inval.

G. mutica Hampe

S. rivulare (Brid.) Podp. ssp.

Grimulare flexifolia Hampe - .

BM(Bremer 1980)]

$\begin{array}{llllllllllllll}\star & 0 & 0 & 0 & 0 & 0 & 0 & 0 & 0 & 0 & 0 & 0 & 0 & 0\end{array}$

$\begin{array}{llllllllllllllll}0 & 0 & 0 & 0 & 0 & 0 & - & 0 & 0 & 0 & 0 & 0 & 0 & 0 & V\end{array}$

$\circ \quad 0 \quad 0 \quad 0 \quad \bullet \quad \bullet \quad 0 \quad 0 \quad 0 \quad 0 \quad 0 \quad 0 \quad 0 \quad$ QVTWA SHemis,

Amer 


\section{GIGASPERMACEAE}

GIGASPERMUM Lindb.

\section{EPHEMERACEAE}

EPHEMERUM Hampe

E. cristatum (Hook. f. \& Wils.) C. Muell.

\section{FUNARIACEAE}

\section{FUNARIA Hedw.}

F. apophysata (Tayl.) Broth.

[Entosthodon apophysata (Tayl.) Mitt.]

F. aristata Broth.

[Entosthodon aristatus (Broth.) Par.]

F. cuspidata Hook. f. \& Wils.

F.glabra Tayl.

[F. crispula Hook. f. \& Wils.

F. tasmanica C. Muell. \& Hampe,

F. subnuda Tayl.]

F.gracilis (Hook. f. \& Wils.) Broth.
[Entosthodon gracilis Hook. f. \& Wils.

F. helmsii Broth. in Geh.

F. hygrometrica Hedw.

F. calvescens Schwaegr.,

F. hygrometrica Hedw. var. sphaerocarpa C.

Muell., F. sphaerocarpa C. Muell.]

F. smithhurstii Broth. \& Geh

[Entosthodon smithhurstii (Broth. \& Geh)

[Entostho
Par.]

Far. squarrifolia Broth.

[Entosthodon squarrifolius (Broth.) Par.]

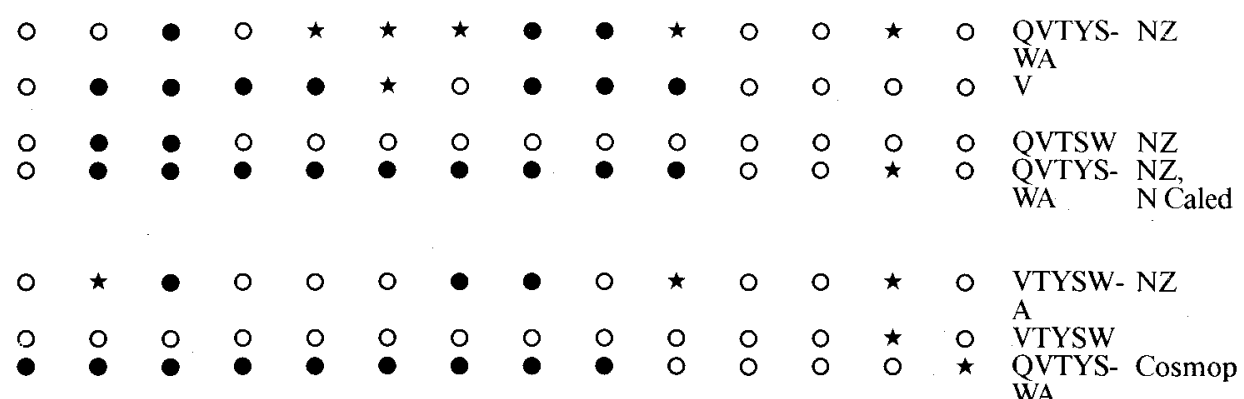

GONIOMITRIUM Hook. f. \& Wils.

G. acuminatum Hook. \& Wils. $\quad$. $\quad \ldots \quad \ldots$

G. ener:e Hook. \& Wils. 


\begin{tabular}{|c|c|c|c|c|c|c|c|c|c|c|c|c|c|c|c|c|}
\hline \multirow[b]{2}{*}{ 19. FUNARIACEAE (cont'd) } & \multirow{2}{*}{$\underset{\mathbf{I}}{\mathbf{H}}$} & \multicolumn{3}{|c|}{ C } & \multicolumn{3}{|c|}{$\mathbf{T}$} & \multicolumn{3}{|c|}{ WS } & \multicolumn{2}{|c|}{ WP } & \multicolumn{2}{|c|}{ FWP } & \multirow{2}{*}{$\begin{array}{l}\text { EXTRA } \\
\text { NSW }\end{array}$} & \multirow{2}{*}{$\begin{array}{l}\text { EXTRA } \\
\text { AUST }\end{array}$} \\
\hline & & $\mathbf{N}$ & C & $\mathbf{s}$ & $\mathbf{N}$ & $\mathrm{C}$ & $\mathbf{S}$ & $\mathbf{N}$ & $\mathrm{C}$ & $\mathbf{S}$ & $\mathbf{N}$ & $\mathbf{S}$ & $\mathbf{N}$ & $\mathbf{S}$ & & \\
\hline
\end{tabular}

PHYSCOMITRELLA B.S.G.

Physcomitrella readeri (C. Muell.) Stone \&

Scott
$[P$. patens (Hedw.) B.S.G. var readeri

(C. Muell.) Tan]

\section{PHYSCOMITRIUM (Brid.) Fuernr.}

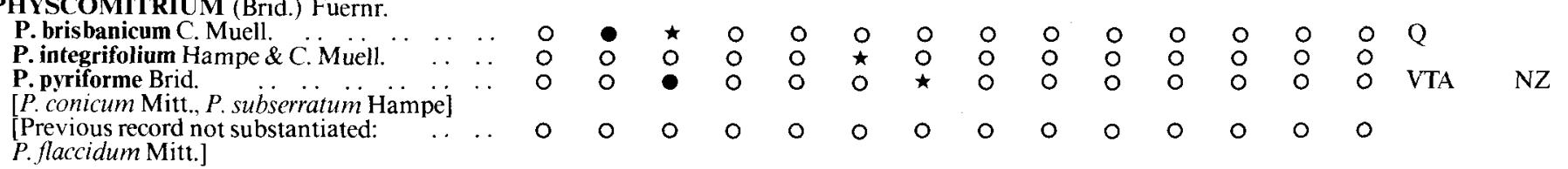

\section{SPLACHNACEAE}

\section{SPLACHNOBRYUM C. Muell.}

S. wattsii Broth.

TAYLORIA Hook.

T. maidenii Broth.

$\begin{array}{llllll}\text { T. octoblepharum (Hook.) Mitt. } & & \ldots & \ldots & \ldots & 0 \\ & & & \end{array}$

T. purpurascens (Hook. \& Wils.) Broth.

0

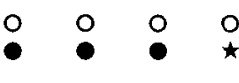

०

00
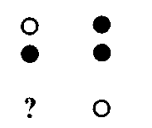

\section{BRYACEAE}

\section{BRACHYMENIUM Schwaegr.}

B. coarctatum Bosch. \& Lac.

B. exile (Dozy \& Molk.) Bosch. \& Lac. $\quad \ldots \quad$.

B. preissianum (Hampe) Jaeg.

B. chloroblastum Watts \& Whitel.

nom. nud.. B. pilosithecium Watts \& Whitel.

00

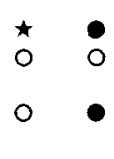

0
$\star$
0

$\begin{array}{ll}0 & 0 \\ 0 & 0 \\ 0 & 0\end{array}$

0

$\star$

$\begin{array}{ll}0 & 0 \\ 0 & 0 \\ 0 & 0\end{array}$

0

0
0
0

0
0
0

$\begin{array}{ll}O & \text { TA } \\ 0 & \text { A }\end{array}$

$\mathrm{NZ}$

As, Oc,

Malesia QVTS- NZ, As, nom mist 
BRYUM Hedg.

B. albo-limbatum (Hampe) Jaeg.

B. cf altisetum C. Muel

B. argenteum Hedw. var. lanatum (P. Beauv.) Hampe

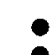

B. argenteum Hedw. var. niveum Wils.

B. australe Hampe.

B. appressifolium Broth 1

B. bateae $C$ Muell.

$\cdots \ldots \ldots$

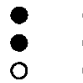

$\begin{array}{lll}0 & 0 & 0 \\ 0 & 0 & 0 \\ 0 & \star & 0\end{array}$

B. billardieri Schwaegr. var. billardieri

$\circ \quad 0 \quad 0 \quad \star$

[B. abruptinervium C. Muell.. B. brachyaris C. Muell. in Mitt. nom. nud.

$B$. breviramulosum (Hampe) Mitt., B. brunneidens, C. Muell., B

crenatidens C. Muell. nom. nud., B. crispatum (Hampe) Mitt., nom.

illeg., B. forsythii Broth., B. globulare Hampe in C. Muell. nom. nud., $B$

leptothecium Tayl, B. olivaceum (Hampe) Mitt., B. pohliaeopsis C.

Muell., B. robustum Hampe, B. rufescens Hook. f. \& Wils. nom. illeg.

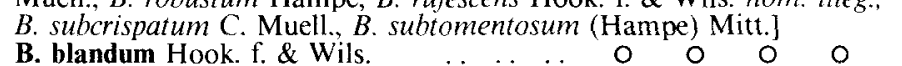

B. blandum Hook. f. \& Wils
[B. oblongifolium $\mathrm{C}$. Muell.]

B. caespiticium Hedw.

B. laxirete Broth.]

B. calodictyon Broth.

B. campylothecium Tayl.

$0 \quad 0 \star 0$

0

$\star$

0

$\cdots+\cdots$

$\begin{array}{lllll}0 & 0 & 0 & 0 & 0\end{array}$

B . peraristatum C. Muell.,

B. howeanum Broth. \& Watts

Brachymenium peraristatum (C. Muell.)

Kindb.]

B. capillare Hedw.

B. immarginatum Broth., B. leptothecioides

Broth. \& Watts nom nud. B pusillum Broth.

nom. illeg., B. sublonginervium Geh. in Watts

\& Whitel. nom nud., B. whiteleggei Broth.

nom. nud.]

B. capillare Hedw. ssp. torquescens (De Not.) $\mathrm{Kindb}$.

[?B. synoicum, C. Muell., B. pyrothecium $\mathrm{C}$.

B. cellulare Hook

B. chrysoneuron $\mathrm{C}$. Muell

B. leptopelma C. Muell.,B lochoneuron $\mathrm{C}$

Muell., B. microthecium C. Muell., $B$.

subervhrocarpum C. Muell., B. wattsii Broth.]

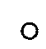

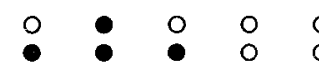

$\begin{array}{cccccc}0 & 0 & 0 & 0 & 0 & 0 \\ 0 & 0 & 0 & 0 & 0 & 0 \\ 0 & \star & 0 & 0 & 0 & 0 \\ \star & 0 & 0 & \star & 0 & 0\end{array}$

O QSW

O VA

QVTYS- Cosmop WA

Cosmop

O VTA

NZ, Eur, $\mathrm{S}$ Amer
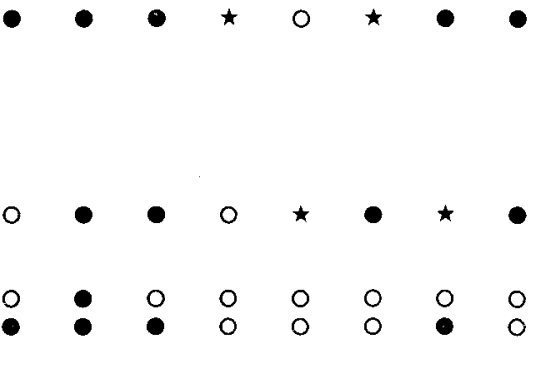

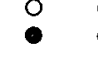

O

O

O 


\begin{tabular}{|c|c|c|c|c|c|c|c|c|c|c|c|c|c|c|c|c|}
\hline \multirow[b]{2}{*}{ 24. BRYACEAE (cont'd) } & \multirow{2}{*}{$\begin{array}{l}\mathbf{L} \\
\mathbf{H} \\
\mathbf{I}\end{array}$} & \multicolumn{3}{|c|}{ C } & \multicolumn{3}{|c|}{$T$} & \multicolumn{3}{|c|}{ WS } & \multicolumn{2}{|c|}{ WP } & \multicolumn{2}{|c|}{ FWP } & \multirow{2}{*}{$\underset{\text { NSW }}{\text { EXTRA }}$} & \multirow{2}{*}{$\begin{array}{c}\text { EXTRA } \\
\text { AUST }\end{array}$} \\
\hline & & $\mathbf{N}$ & C & $\mathbf{S}$ & $\mathbf{N}$ & C & $\mathbf{S}$ & $\mathbf{N}$ & C & $\mathbf{s}$ & $\mathbf{N}$ & $\mathbf{S}$ & $\mathbf{N}$ & $\mathbf{S}$ & & \\
\hline \multicolumn{17}{|l|}{ BRYUM (cont'd) } \\
\hline $\begin{array}{l}\text { B. coronatum Schwaegr. } \\
\text { [B. subatropurpureum } \text { C. Muell. }]\end{array}$ & $\bullet$ & - & - & $\star$ & 0 & $\bullet$ & $\star$ & 0 & - & $\circ$ & 0 & 0 & 0 & $\circ$ & QWA & Cosmop \\
\hline $\begin{array}{l}\text { B. crassum Hook. f. \& Wils. } \\
\text { [B. austro-alpinum C. Muell.] }\end{array} \quad \cdots \quad \cdots \quad \cdots$ & 0 & 0 & $\star$ & 0 & 0 & $\circ$ & 0 & 0 & 0 & 0 & 0 & $\circ$ & 0 & o & VT & NZ \\
\hline $\begin{array}{l}\text { B. creberrimum Tayl. } \\
\text { [B. affine Lindb. \& Arn. nom. illeg., } \\
\text { B. tasmanicum Hampe] }\end{array}$ & $\circ$ & $\star$ & $\star$ & $\star$ & o & $\star$ & $\bullet$ & $\circ$ & $\star$ & $\circ$ & 0 & $\circ$ & 0 & $\circ$ & VSW & $\begin{array}{l}\text { NZ,N } \\
\text { Amer, } \\
\text { Eur, As, }\end{array}$ \\
\hline $\begin{array}{llllll}\text { B. cupulatum C. Muell. } & \ldots & \ldots & \ldots & \ldots & \ldots \\
\text { B. dichotomum Hedw. } & \ldots & . . & \ldots & \ldots & . .\end{array}$ & $\stackrel{9}{0}$ & $\stackrel{\circ}{\circ}+\frac{1}{\circ}+1$ & $:$ & $\begin{array}{l}0 \\
0\end{array}$ & $\stackrel{\circ}{\circ}$ & $\stackrel{-1}{0}$ & $\stackrel{\circ}{\star}$ & $\stackrel{\circ}{\circ}+\frac{1}{\circ}+1$ & $\begin{array}{l}\circ \\
\circ\end{array}$ & $\stackrel{\circ}{\star}$ & $\begin{array}{l}\circ \\
\circ\end{array}$ & 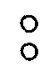 & $\stackrel{\circ}{\star}$ & $\stackrel{\circ}{\circ}$ & $\begin{array}{l}\text { VTS } \\
\text { QVTSA }\end{array}$ & NZ, Amer, \\
\hline $\begin{array}{l}\text { [B. aequicollum Broth. \& Watts, } \\
\text { B. brachytheciella C. Muell., } \\
\text { B. philonotideum Broth. \& Watts, } \\
\text { B. pimpamae C. Muell., } \\
\text { B. subcupulatum C. Muell. ex Rodw.] }\end{array}$ & & & & & & 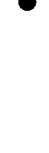 & & & & & & & & & & Subant I \\
\hline B. diversinerve Broth. \& Watts & $\bullet$ & $\circ$ & 0 & $\circ$ & o & o & $\circ$ & $\circ$ & $\circ$ & $\circ$ & $\circ$ & $\circ$ & $\circ$ & $\circ$ & & \\
\hline $\begin{array}{l}\text { B. erythrocarpoides C. Muell. \& Hampe } \\
\text { [B. clavatum Hook. f. \& Wils., B. curvicollum } \\
\text { Mitt.,B. filarium Broth., B. kiamae Broth., } \\
\text { B. subcurvicollum Broth.] }\end{array}$ & - & - & - & $\circ$ & $\star$ & $\star$ & - & 0 & $\star$ & 0 & 0 & 0 & 0 & 0 & QVTSA & $\begin{array}{l}\text { NZ, S Afr, } \\
\text { Oc }\end{array}$ \\
\hline B. laevigatum Hook. f. \& Wils. $\quad \ldots \quad \ldots \quad \ldots$ & o & $\star$ & $\circ$ & $\circ$ & 0 & $\circ$ & $\bullet$ & $\circ$ & 0 & $\star$ & $\mathrm{o}$ & 0 & o & 0 & VTA & $\begin{array}{l}\text { NZ, S Afr, } \\
\text { S Amer, } \\
\text { Subant I }\end{array}$ \\
\hline $\begin{array}{l}\text { B. leucocanthum (Hampe) Mitt. } \\
\text { [Rhodobryum leucocanthum Hampe] }\end{array}$ & - & 0 & 0 & o & 0 & $\circ$ & 0 & 0 & 0 & 0 & 0 & 0 & 0 & 0 & & \\
\hline B. limbifolium Broth. \& Watts $\quad$. . . . & - & 0 & o & $\mathrm{o}$ & 0 & 0 & 0 & $\mathrm{O}$ & 0 & 0 & 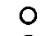 & & & 0 & & \\
\hline $\begin{array}{l}\text { B. muehlenbeckii B.S.G. } \\
{\left[\begin{array}{lllll} \\
\text { B. cheelii Broth.] }\end{array}\right.}\end{array}$ & o & o & $\bullet$ & o & 0 & o & $\star$ & 0 & 0 & $\circ$ & o & & o & 0 & A & Cosmop \\
\hline $\begin{array}{l}\text { B. nitens Hook. } \\
\text { [B. baileyi Broth., B. pachypomatulum Broth., } \\
\text { B. subpachypoma Hampe, B. } \\
\text { micropachypoma Broth. nom. nud.] }\end{array}$ & o & - & $\star$ & $\circ$ & 0 & $\star$ & 0 & 0 & 0 & 0 & 0 & $\circ$ & 0 & o & Q & As, Afr, Oc \\
\hline $\begin{array}{l}\text { B. pachytheca C. Muell. } \\
\text { [B. bicolor Dicks., B. gambierense C. Muell., } \\
\text { B. microerythrocarpum C. Muell. \& Kindb., } \\
\text { B. subaeneum Hampe \& C. Muell., } \\
\text { B. sullivanii C. Muell.] }\end{array}$ & $\mathrm{o}$ & - & $\bullet$ & $\star$ & 0 & $\star$ & $\bullet$ & o & $\bullet$ & $\star$ & • & $\circ$ & $\star$ & $\circ$ & $\begin{array}{l}\text { QVTYS- } \\
\text { WA }\end{array}$ & Cosmop \\
\hline $\begin{array}{llllllll}\text { B. cf. pallens Sw. } & \ldots & \ldots & \ldots & \ldots & \ldots & \ldots & \end{array}$ & 0 & $\circ$ & $\circ$ & $\circ$ & O & $\mathrm{o}$ & $\star$ & $\circ$ & $\circ$ & $\circ$ & $\circ$ & $\circ$ & $\circ$ & $\circ$ & A & \\
\hline $\begin{array}{lllll}\text { B. perlimbatum Card. } \quad \text {.. } & \ldots & \ldots\end{array}$ & 0 & 0 & 0 & 0 & 0 & 0 & $\star$ & 0 & 0 & 0 & $\mathrm{O}$ & $\mathrm{o}$ & 0 & 0 & & S Amer \\
\hline
\end{tabular}

B. clavatum Hook. f. \& Wils., B. curvicollum

Mitt. B. filarium Broth., B. kiamae Broth

B. subcurvicollum Broth.

B. leucocanthum (Hampe) Mitt.

[Rhodobryum leucocanthum Hampe]

B. limbifolium Broth. \& Watts

B. baileyi Broth. B. pachypomatulum Broth

$B$. subpachypoma Hampe, $B$.

micropachypoma Broth. nom. nud.]

theca C. Muell.

B. perlimbatum Card.

$\begin{array}{llll} & \cdots & \ldots & \ldots\end{array}$ 
B. plumosum Dozy \& Molk

[B. pachypoma Mont.]

B. pseudotriquetrum (Hedw.) Gaertn.

B. austro-affine Broth.

B. bimum (Brid.) Turn.,

B. subventricosum Broth.]

B. roseodens C. Muell.

B. sauteri B.S.G.

B. subapiculatum Hampe

B. subfasciculatum (Hampe) Mitt.

B. amoenum Wright non Broth. $n$

B. dilatato-marginatum C. Muell.,

Rhodobryum subfasciculatum, Hampe,

Bryum subolivaceum C. Muell.

?B. viridulum C. Muell. nom. illeg.]

[Previous record not substantiated

B. baeuerlenii C. Muell., B. brachvcladulum $\ddot{C}$.

Muell., B. ischyrrodon C. Muell.]

Wils.

LEPTOBRYUM (B.S.G.) Wils

L. pyriforme (Hedw)

MIELICHHOFERIA Nees \& Hornsch

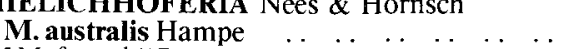

[M. forsvthii Broth.]

[M. ecklonii Hornsch.]

M. turgens Broth.

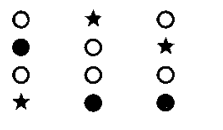

o 00

O

00

00000

- $\mathrm{O}$

o 0

$\circ 0$

VTSWA Cosmop

o

0
0
0
$\star$

$\begin{array}{lll}0 & 0 & 0 \\ 0 & 0 & 0 \\ 0 & \star & 0 \\ 0 & 0 & 0\end{array}$

$\begin{array}{ll}0 & 0 \\ 0 & 0 \\ 0 & 0 \\ 0 & 0\end{array}$

$\begin{array}{lll}0 & 0 & 0 \\ 0 & 0 & 0 \\ 0 & 0 & 0 \\ 0 & 0 & 0\end{array}$

$\begin{array}{lllll}0 & 0 & 0 & 0 & \\ 0 & 0 & 0 & 0 & V \\ 0 & 0 & 0 & 0 & A \\ 0 & 0 & 0 & 0 & \end{array}$

S Amer

NZ, Eur

Oc

RTHODONTIUM Schwaegr.

O. inflatum (Mitt.) Par.

IO, ovale C. Muell. ex Broth.,

Wilsoniella compacta Geh.in Watts \& Whitel.

nom. nud.]

O. lineare Schwaegr. ssp. sulcatum (Hook. f.

\& Wils.) Meijer

1O. australe Hook. f. \& Wils., O. sulcatum

Hook. f. \& Wils., $O$. pallens (Hook. f. \& Wils.)

Broth., O. zetterstedtii C. Muell., Aplodium

lineare Mitt. nom. illeg.] 


\begin{tabular}{|c|c|c|c|c|c|c|c|c|c|c|c|c|c|c|c|c|c|c|}
\hline \multirow[b]{2}{*}{ 24. BRYACEAE (cont'd) } & & & \multirow{2}{*}{$\underset{\mathbf{H}}{\mathbf{L}}$} & \multicolumn{3}{|c|}{ C } & \multicolumn{3}{|c|}{$\mathbf{T}$} & \multicolumn{3}{|c|}{ WS } & \multicolumn{2}{|c|}{$\mathbf{W P}$} & \multicolumn{2}{|c|}{ FWP } & \multirow{2}{*}{$\underset{\text { NSW }}{\text { EXTRA }}$} & \multirow{2}{*}{$\begin{array}{l}\text { EXTRA } \\
\text { AUST }\end{array}$} \\
\hline & & & & $\mathbf{N}$ & $\mathbf{C}$ & $\mathbf{S}$ & $\mathbf{N}$ & $\mathbf{C}$ & $\mathbf{S}$ & $\mathbf{N}$ & $\mathbf{C}$ & $\mathbf{S}$ & $\mathbf{N}$ & $\mathbf{S}$ & $\mathbf{N}$ & $\mathbf{S}$ & & \\
\hline \multicolumn{19}{|l|}{ POHLIA Hedw. } \\
\hline P. bulbifera (Warnst.) Warnst. & .. & & 0 & O & $\mathrm{O}$ & 0 & $\mathrm{O}$ & 0 & $\star$ & 0 & 0 & 0 & 0 & 0 & 0 & 0 & \multirow{5}{*}{$\begin{array}{l}\text { VTS } \\
\text { V } \\
\text { VTS?W- } \\
\text { ?A }\end{array}$} & N Hemis \\
\hline $\begin{array}{l}\text { P. cruda (Hedw.) Lindb. } \\
{[P \text {. ervthrocaulis Watts \& Whitel.] }}\end{array}$ & $\cdots$ & . & 0 & 0 & 0 & 0 & 0 & $\star$ & $\star$ & 0 & 0 & 0 & 0 & 0 & 0 & $\mathrm{O}$ & & $\begin{array}{l}\text { NZ, } \\
\text { Cosmop }\end{array}$ \\
\hline P. mielichhoferia (C. Muell.) Broth. & & . & 0 & 0 & 0 & 0 & 0 & 0 & - & 0 & 0 & 0 & 0 & 0 & 0 & 0 & & \\
\hline $\begin{array}{l}\text { P. nutans (Hedw.) Lindb. } \\
\text { [Webera nutans Hedw.] }\end{array}$ & $\ldots$ & . & 0 & $\mathrm{O}$ & $\star$ & 0 & $\star$ & - & $\bullet$ & 0 & O & 0 & 0 & o & 0 & $\mathrm{O}$ & & Cosmop \\
\hline $\begin{array}{l}\text { P. tenuifolia (Jaeg.) Broth. } \\
\text { [Webera tenuifolia Jaeg.] }\end{array}$ & $\cdots$ & . & 0 & 0 & $\star$ & 0 & $\mathrm{O}$ & 0 & $\mathrm{O}$ & 0 & 0 & 0 & 0 & 0 & 0 & 0 & & $\mathrm{NZ}$ \\
\hline \multicolumn{3}{|c|}{$\begin{array}{l}\text { P. wahlenbergii (Web. \& Mohr) Andrews } \\
\text { [Mniobryum wahlenbergii (Web. \& Mohr) } \\
\text { Jenn., M. tasmanicum Broth., M. albicans } \\
\text { (Wahlenb.) Limpr. }\end{array}$} & 0 & 0 & 0 & 0 & - & 0 & $\bullet$ & 0 & $\mathrm{O}$ & $\star$ & 0 & $\mathrm{O}$ & 0 & 0 & VTA & Cosmop \\
\hline
\end{tabular}

\section{RHODOBRYUM (Schimp.) Hampe}

R. aubertii (Schwaegr.) Thér.

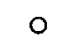

00

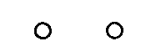

○

$\circ \quad \circ \quad \circ \quad 0 \quad 0$

S Afr,

S Amer,

Madagas-

R. graeffeanum (C. Muell.) Par.

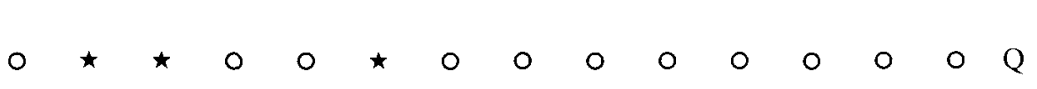

\section{LEPTOSTOMATACEAE}

\section{LEPTOSTOMUM R. Br.}

L. erectum R. Br.

L. inclinans $\mathrm{R}$. Br.

$\begin{array}{llllll}\cdots & \cdots & \cdots & \cdots & \ldots & 0 \\ & & \cdots & \end{array}$

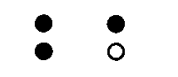

L. macrocarpum (Hedw.) Pyl.

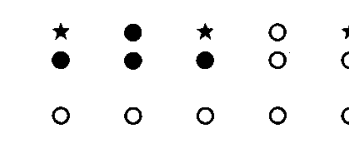

$\stackrel{0}{\circ}$

\section{MNIACEAE}

PLAGIOMNIUM T. Kop.

P. novae-zealandiae (Col.) T. Kop.

Mnium longirostrum Brid.

$M$. novae-valesiae C. Muell. in Geh.

$M$. rostratum auct. non Schrad.] 


\section{MITTENIACEAE}

\section{MITTENIA Lindb.}

M. plumula (Mitt) Lindb.

[M. rotundifolia (C. Muell.) Par.]

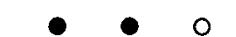

\section{Calomniaceae}

CALOMNION Hook. f. \& Wils.

C. complanatum (Hook. f. \& Wils.) Lindb.

[C. laetum Hook. f. \& Wils. nom. illeg.]

0

- 0

0

0

0000

NZ

32. RHIZOGONIACEAE
GONIOBRYUM Lindb
G. subbasilare (Hook.) Lindb.
[G. pellucidum (Mitt.) Broth.]
. . . .
o
- 00

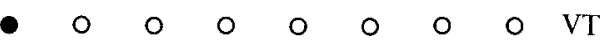
$\mathrm{NZ}, \mathrm{Oc}$
HYMENODON Hook. f. \& Wils.
H. pilifer Hook. f. \& Wils.

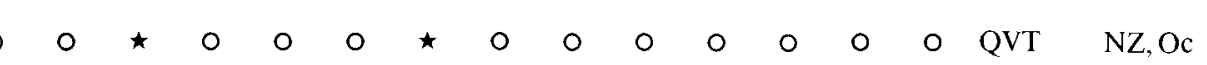
LEPTOTHECA Schwaegr. $\dagger$
L. gaudichaudii Schwaegr. var. gaudichaudii
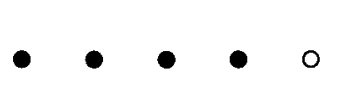
$\star \quad 0$
$\circ \quad 0 \quad 0 \quad 0 \quad$ QVTA
MESOCHAETE Lind.
M. undulata Lindb.

\section{PYRRHOBRYUM Mitt.}
P. mnioides (Hook.) Manuel
[Rhizogonium mnioides (Hook.) Wils. in
Hook.]
P. parramattense (C. Muell.) Manuel
[Rhizogonium parramattense(C. Muell.)
Reichdt.]
P. spiniforme (Hedw.) Manuel
[Rhizogonium spiniforme Hedw.]

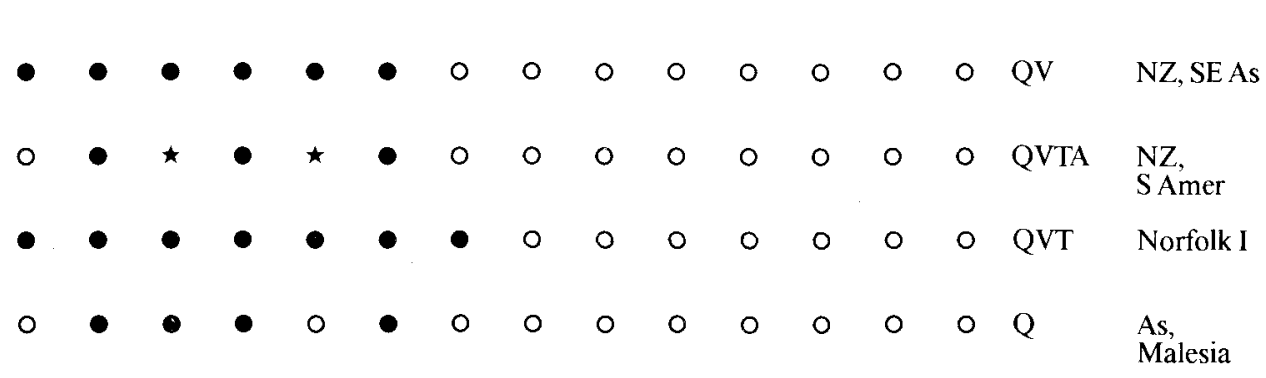

$\uparrow$ See Churchill \& Buck (1982). 


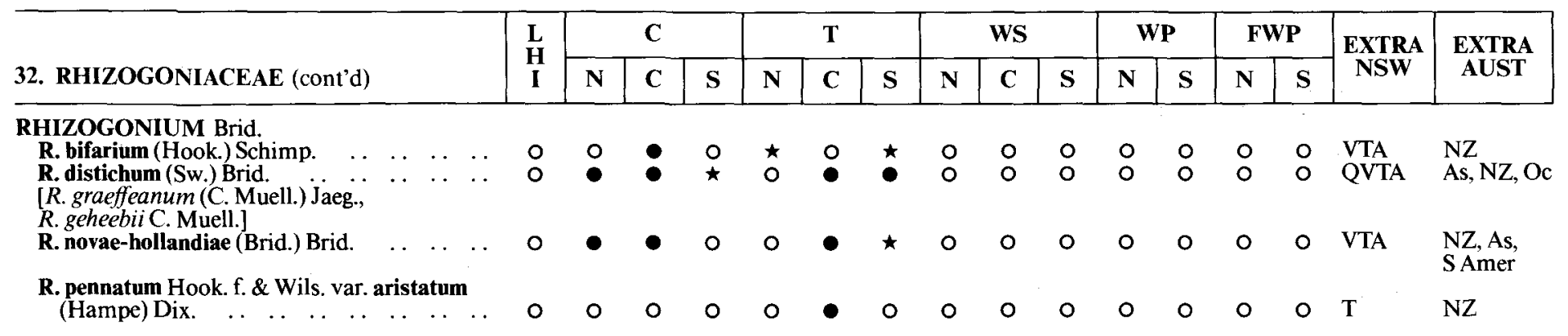

\section{HYPNODENDRACEAE}

\section{BRAITHWAITEA Lindb.}

B. sulcata (Hook.) Jaeg.

(1)
HYPNODENDRON (C. Muell.) Lindb. ex Mitt.
H. colensoi (Hook. f. \& Wils.) Mitt. $\quad . . \quad \ldots$

[Known only from a specimen in NY (Touw

1971)]

H. comosum (Labill.) Mitt.

[Mniodendron comosum (Labill.) Lindb.]

H. spininervium (Hook.) Jaeg. ssp. archeri

(Mitt.) Touw .. . .

[H. arcuatum Hedw. emend. Mitt. pro parte]

H. vitiense Mitt. ssp. australe Touw ..
$[H$. whiteleggei $\mathrm{C}$. Muell. ex Burges nom.

$H$. arcuatum non Hedw.

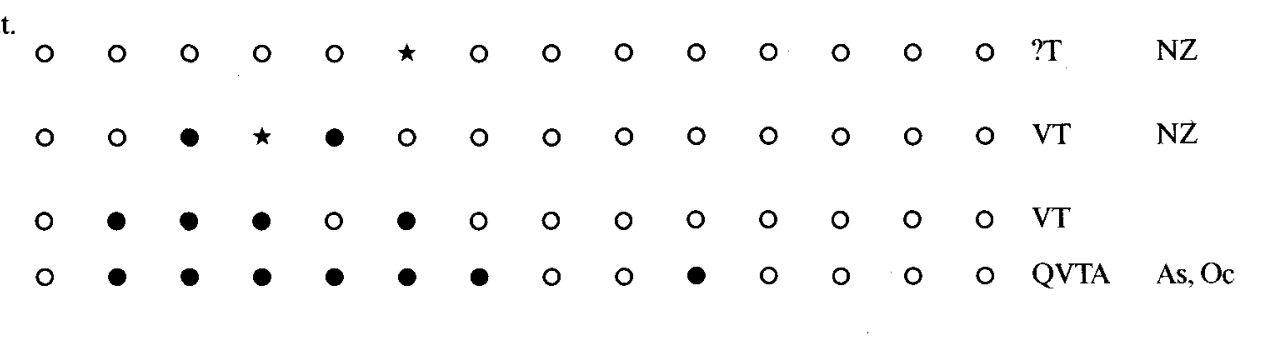

34. AULACOMNIACEAE

AULACOMNIUM Schwaegr.

A. palustre (Hedw.) Schwaegr. 
35. MEESIACEAE

MEESIA Hedw.

$\begin{array}{llllllllllllllllllllllllll}\text { M. muelleri C. Muell. \& Hampe } & \ldots & \ldots & \ldots & 0 & 0 & 0 & 0 & 0 & 0 & 0 & 0 & 0 & 0 & 0 & 0 & 0 & 0 & V A & N Z & \\ \text { M. triquetra (Hook. \& Tayl.) Aongstr. } & \ldots & \ldots & 0 & 0 & 0 & 0 & 0 & 0 & 0 & 0 & 0 & 0 & 0 & 0 & 0 & 0 & \text { VA } & \text { N Hemis }\end{array}$

\section{BARTRAMIACEAE}

\section{BARTRAMLA Hedw.}

B halleriana Hedw.

[B. mossmanniana C. Mueli.

B. hampei (Mitt.) Catcheside

[Bartramidula hampei Mitt.,

Bartramia erecta (Hampe) Broth. nom. illeg.]

B. papillata Hook. f. \& Wils. .. .. ..

$$
\begin{array}{cccccccccccccccc}
0 & 0 & 0 & 0 & 0 & 0 & \bullet & \star & 0 & 0 & 0 & 0 & 0 & 0 & V T & \text { NZ, } \\
0 & 0 & 0 & 0 & \star & 0 & \bullet & 0 & \bullet & 0 & 0 & 0 & 0 & 0 & \text { VTSWA } &
\end{array}
$$

B. stricta Brid

[B. strictifolia Tayl.

(not the same as $\mathrm{N}$ Hemis sp. - under review

by Catcheside)

B. timmioides C. Muell.

[Known only from type in $\dot{B M}$ ]

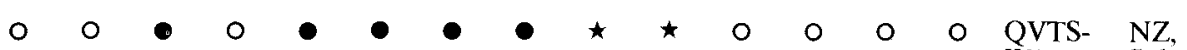

REUTELIA (B.S.G.) Schimp.

B. affinis (Hook.) Mitt.
$[B$. commutata Jaeg. nom. illeg., Philonotis

affinis (Hook.) Jaeg.]

B. pendula $(\mathrm{Sm}$. $)$ Mitt.

B. divaricata (Mitt.) Mitt.,

$B$. fusco-aurea Broth.,

B. sieberi (Hornsch. ex C. Muell.) Mitt.]

B. sieberi (Hornsch. ex C. Muell.) Mitt.]
B. pseudo-philonotis (C. Muell.) Watts \&

Whitel.

[Previous record not substantiated:

B. baeuerlenii (C. Muell.) Watts \& Whitel.

B. leptodontoides (C. Muell.) Watts \& Whitel.

B. lonchopelma (C. Muell.) Watts \& Whitel.]

$\begin{array}{llllllllllllll}0 & 0 & 0 & 0 & 0 & 0 & 0 & 0 & 0 & 0 & 0 & 0 & 0 & 0 \\ 0 & 0 & 0 & 0 & 0 & 0 & 0 & 0 & 0 & 0 & 0 & 0 & 0 & 0\end{array}$

CONOSTOMUM Swartz

$\begin{array}{lllll}\text { C. curvirostre (Mitt.) Mitt. } & \ldots & \ldots & \ldots & \ldots \\ \text { C. pentastichum (Brid.) Lindb. } & & \ldots & \ldots & \ldots\end{array}$

[C. australe $\mathrm{Sw}$.

C. pusillum Hook. f. \& Wils.

- 0

$\star \quad 0$

WVTS- NZ,

(1)

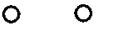

O

0

o

o

० 0 O 0 QTWA

Subant I

.

$\begin{array}{ll}0 & 0 \\ 0 & 0 \\ 0 & 0\end{array}$

$\begin{array}{ll}0 & 0 \\ 0 & 0 \\ 0 & 0\end{array}$

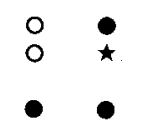

0
0
0

$\begin{array}{ll}0 & 0 \\ 0 & 0 \\ 0 & 0\end{array}$

0

0
0
0

0

$\begin{array}{lll}0 & 0 & \text { VA } \\ 0 & 0 & \text { VTA } \\ & & \end{array}$

NZ, S Afr, SAmer

NZ 


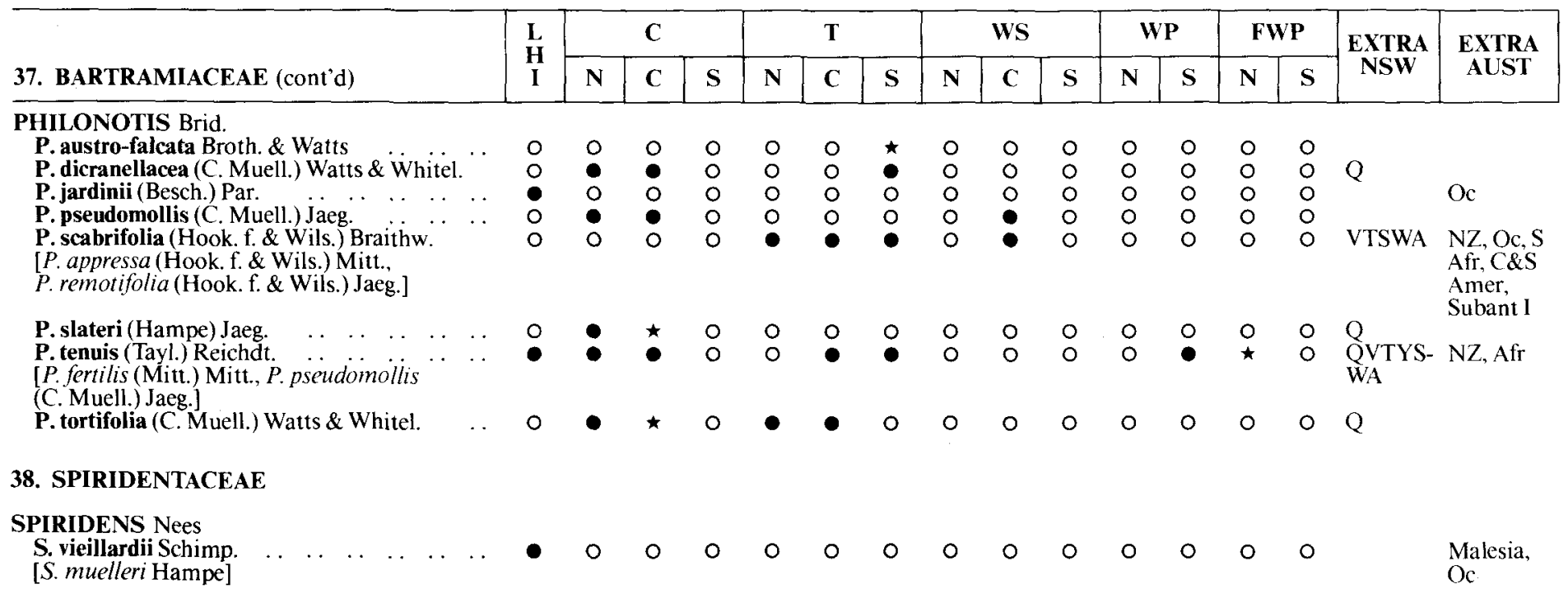

\section{ERPODIACEAE}

\section{AULACOPILUM Wils. \\ A. glaucum Wils.}

A. hodgkinsoniae (Hampe $\ddot{\&} \dot{C}$. Mueli.)

Broth.

41. PTYCHOMITRIACEAE $\dagger$

\section{PTYCHOMITRIUM Fuernr. \\ P. australe (Hampe) Jaeg.}

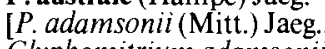

Glyphomitrium adamsonil Mitt.

Brachysteleum australe (Hampe) Jaeg.I

$\uparrow$ Transferred to Grimmiaceae by Churchill (1982). 
P. laxifolium (C. Muell.) Par.

[Brachysteleum laxifolium C Muell]

$0 \star$

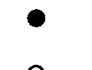

P. mittenii Jaeg

P. serratum (Mitt) . ... ... .

illeg.]

P. muelleri (Mitt.) Jaeg.

[P. commutatum (C. Muell.) Par.

Glyphomitrium muelleri (C. Muell.) Broth.,

$G$. howeanum (Hampe) Broth.]

\section{ORTHOTRICHACEAE}

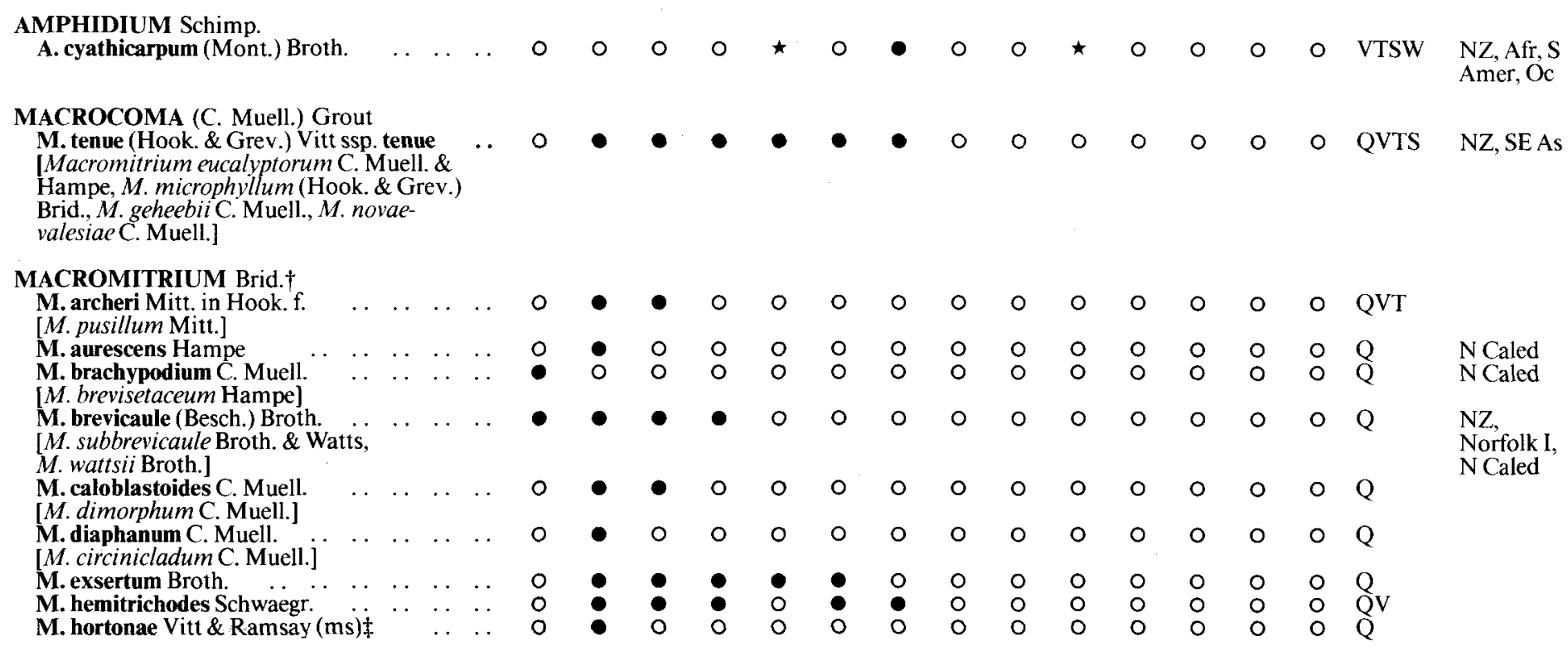

† Revision by Vitt \& Ramsay (manuscript) - only synonyms applicable to NSW collections are included except when clarification with an Australian used name is needed.

$\ddagger$ Manuscript names, nov. sp. or ssp. 


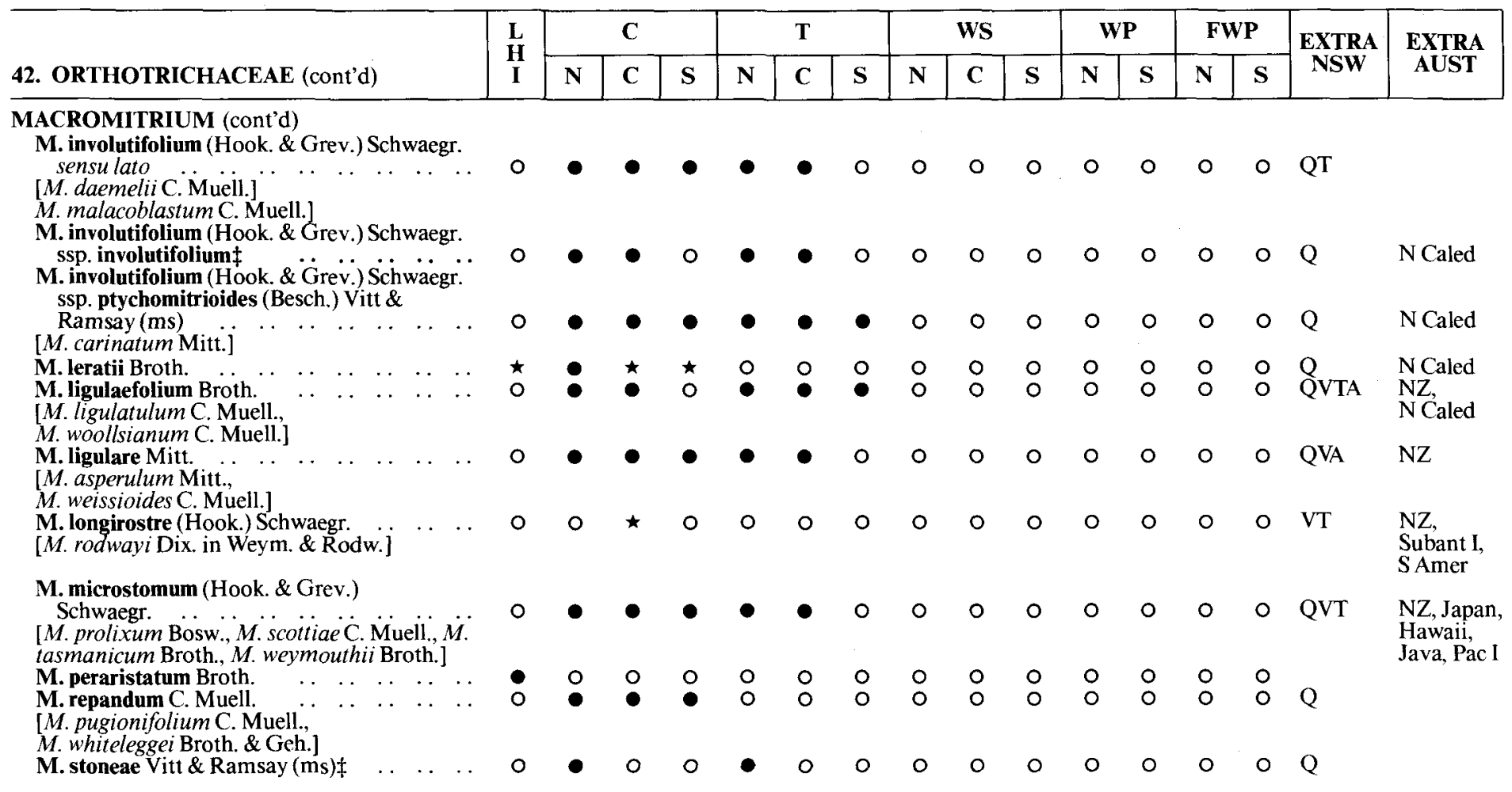


ORTHOTRICHUM Hedw. $†$

O. cupulatum Hoffm. ex Brid. var. cupulatum

O. hortense Bosw.

O. longithecum $\mathrm{R}$. $\mathrm{Br}$. ter

[O. acroblepharis C. Muell.]

O. rupestre Schleich. ex Schwaegr. var.

rupestre

[O. sullivanii $\mathrm{C}$. Mueill.

O. tasmanicum Hook. f. \& Wils. var.

tasmanicum

O. encalyptaceum $\dot{\mathrm{C}}$. Muell., $O$ laterale

Hampe, O. campbelliae Watts \& Whitel.]

SCHLOTHEIMIA Brid.

$\begin{array}{llllllll}\text { S. brownii Schwaegr. } & \ldots & \ldots & \ldots & \ldots & \ldots & \ldots & 0\end{array}$

[S. baileyi Broth.]

S. funiformis Tayl. ex Dix.

ZYGODON Hook. \& Tayl.

Z. hookeri Hampe

Z. intermedius B.S.G.

[Z. brownii Schwaegr.]

Z. menziesii (Schwaegr.) Arnott

$\begin{array}{llllll}\cdots & \cdots & \ldots & \ldots & \ldots & 0 \\ \cdots & \ldots & \ldots & \ldots & \ldots & 0\end{array}$

$\begin{array}{llll}0 & 0 & 0 & 0 \\ 0 & 0 & \star & 0\end{array}$

○

0

$\begin{array}{lll}0 & \bullet & 0 \\ 0 & \bullet & 0 \\ 0 & \star & 0\end{array}$

$\begin{array}{lll}0 & 0 & 0 \\ 0 & 0 & 0\end{array}$

O

○ $\circ \mathrm{VA}$

Eur, As, Afr,

$\mathrm{N}$ Amer

$\begin{array}{lllllllll}0 & 0 & 0 & 0 & 0 & 0 & 0 & V & N A \\ 0 & \bullet & 0 & 0 & 0 & 0 & 0 & V A & N Z\end{array}$

00

$\star \quad \mathrm{O}$

○

VTA

Cosmop

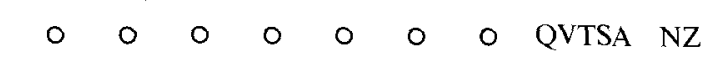

o

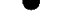

0

- 0

O

NZ

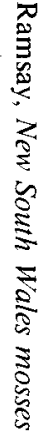

\section{RACOPILACEAE}

RACOPILUM P. Beauv. $\$$

R. cuspidigerum (Schwaegr.) Aongst. var.

cuspidigerum

[R. pacificum Besch.]

R. cuspidigerum (Schwaegr.) Aongst. var.

convolutaceum (C. Muell.) Zant. \&

$$
\begin{aligned}
& \text { Convolutace } \\
& \text { Dijkstra }
\end{aligned}
$$

[R. convolutaeceum (C. Mueli.) Reichdt

$R$. strumiferum auct. non (C. Muell.) Mitt. as

in NSW

+ Determined by $\mathbf{J}$. Lewinsky (in press).

\$ Based on studies by van Zanten \& Dijkstra (unpubl.). 


\begin{tabular}{|c|c|c|c|c|c|c|c|c|c|c|c|c|c|c|c|c|}
\hline \multirow[b]{2}{*}{ 47. HEDWIGIACEAE } & \multirow{2}{*}{$\begin{array}{l}\mathbf{L} \\
\mathbf{H} \\
\mathbf{I}\end{array}$} & \multicolumn{3}{|c|}{ C } & \multicolumn{3}{|c|}{$\mathbf{T}$} & \multicolumn{3}{|c|}{ WS } & \multicolumn{2}{|c|}{ WP } & \multicolumn{2}{|c|}{ FWP } & \multirow{2}{*}{\begin{tabular}{|} 
EXTRA \\
NSWW
\end{tabular}} & \multirow{2}{*}{$\begin{array}{c}\text { EXTRA } \\
\text { AUST }\end{array}$} \\
\hline & & $\mathbf{N}$ & C & $\mathbf{S}$ & $\mathbf{N}$ & $\mathbf{C}$ & $\mathbf{S}$ & $\mathbf{N}$ & C & $\mathbf{s}$ & $\mathbf{N}$ & $\mathbf{S}$ & $\mathbf{N}$ & $\mathbf{S}$ & & \\
\hline
\end{tabular}

\section{HEDWIGIA P. Beauv.}

H. ciliata (Hedw.) Ehrh. ex P. Beauv.

[H. albicans Lindb. nom. illeg., H. juratzkae

C. Muell., H. microcyathea (C. Muell.) Par.]

H. integrifolia P. Beauv.

[Hedwigidium integrifolium (P. Beauv.) Dix.,

0

o

Hedwigidium imberbe (Sm.) B.S.G.]

RHACOCARPUS Lindb.

R. purpurascens (Brid.) Par.

[R. australis (Hook. f. \& Wils.) Par.,

R. humboldtii (Hook.) Lindb.]
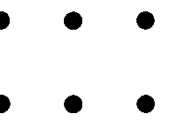
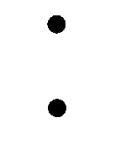

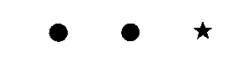

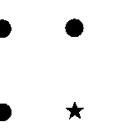

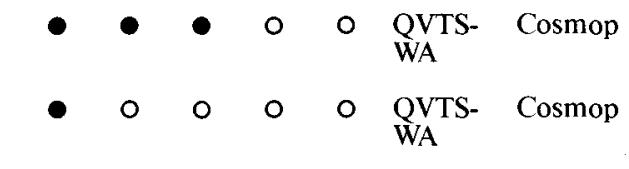

\section{CRYPHAEACEAE}

\section{CRYPHAEA Mohr.}

C. dilatata Hook. f. \& Wils.

[C. muelleri (Hampe) Broth., Cryphidium

muelleri (Hampe) Broth., Cyptodon dilatatus

(Hook. f. \& Wils.) Par. \& Schimp.]

C. exannulata Dix. \& Sainsb.

C. ovalifolia (C. Muell.) Jaeg.

[Cyptodon ovalifolius (C. Muell.) Fleisch

Cryphidium ovalifolium (C. Muell.) Broth.

Cryphaea squarrulosa Hampe, Cryphidium

squarrulosum (Hampe) Broth.]

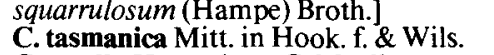

C. tenella Hornsch. ex C. Muell.

[C. brevidens C. Muell.]

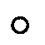

\section{FORSSTROEMIA Lind.}

F. australis (C Muell) $\mathrm{Par}$.

$\begin{array}{llllll}\text { F. australis (C. Muell.) Par. } & \ldots & \ldots & \ldots & \ldots & 0 \\ \text { F. subproducta (C. Muell.) Broth. } & \ldots & \ldots & \ldots & 0\end{array}$

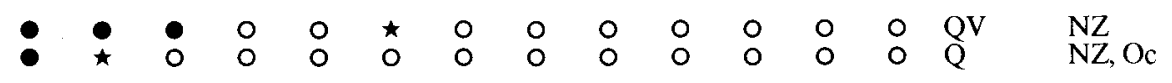

$\begin{array}{lllllllllllll}- & 0 & 0 & 0 & 0 & 0 & 0 & 0 & 0 & 0 & 0 & 0 & Q \\ 0 & 0 & 0 & 0 & 0 & 0 & 0 & 0 & 0 & 0 & 0 & Q\end{array}$

\section{CYRTOPODACEAE}

BESCHERELLIA Duby

B. brevifolia Hampe

[B. cyrtopus C. Muell., $\dot{\text { Cyrtopus }}$

bescherellioides $\mathrm{C}$. Muell.]

$-$ 


\section{PTyChomniaceae}

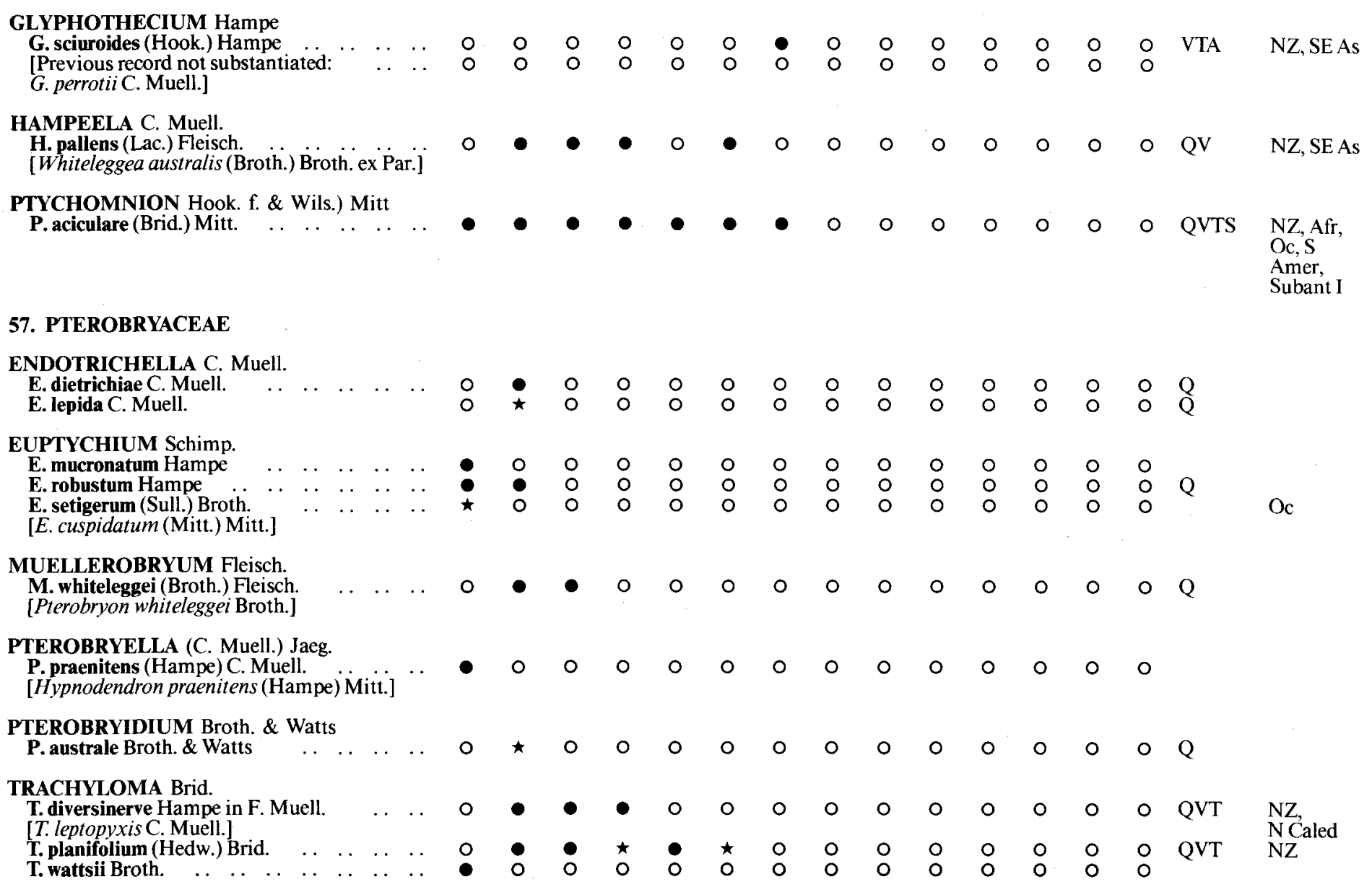




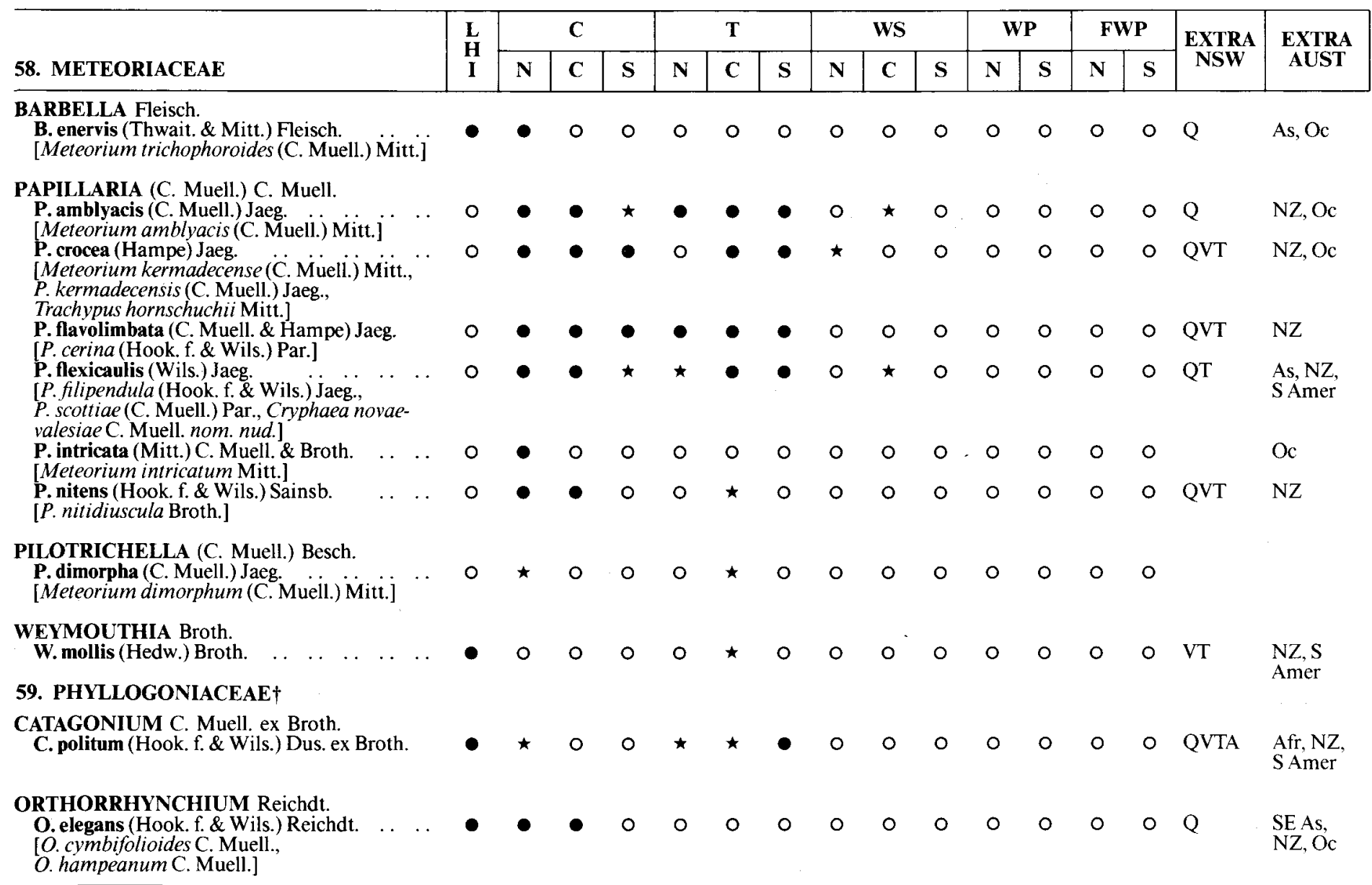

†See Lin (1983) for new classification. 


\section{NECKERACEAE}

\section{CALYPTOTHECIUM Mitt.}

C. acutum (Mitt.) Broth.

C. cuspidatum (Okam.)
C. humile (Mitt.) Broth.

$\begin{array}{lllll} & \cdots & \cdots & \ldots & \ldots\end{array}$

\section{LEPTODON Moh.}

L. smithii (Hedw.) Web. \& Mohr. . . . . . .
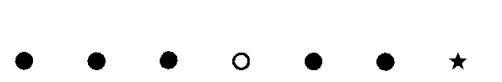

NECKERA Hedw.

N. pennata Hedw.

[N. hymenodonta C. Muell.]

N. leichhardtii Hampe

[Known only from specimen in BM

THAMNOBRYUM Nieuwl.

T. pandum (Hook. f. \& Wils.) Stone \& Scott

(Thamnium pandum (Hook f. \& Wils.) Jaeg.,

Thamnium eflagellare Aongstr.]

T, pumilum (Hook. f. \& Wils.) Nieuwl.

[Thamnium pumilum (Hook. f. \& Wils.)

Kindb., Thamnium gracillimum (Hampe)

Broth.\& Watts, Homalia acuminata C.

Muell.]

[Previous record not substantiated:

T. novae-valesiae Kindb.]

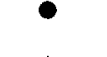

\section{LEMBOPHYLLACEAE}

CAMPTOCHAETE Reichdt.

C. arbuscula (Sm.) Reichdt.

[C. ramulosa (Mitt.) Jaeg., Porotrichum

arbusculum (Sm.) Mitt.]

C. arbuscula (Sm.) Reichdt. var. deflexa

(Wils.) Dix.

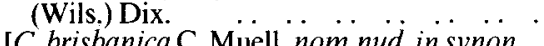

[C. brisbanica C. Muell. nom nud in synon.

C. deflexa (Wils. Jaeg.]

C. excavata (Tayl.) Jaeg.

C. gracilis (Hook. f. \& Wils.) Par.

Lembophyllum brisbanicum C. Muell nom

nud.]

C. leichhardtii (Jaeg.) Broth.
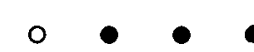

o

0

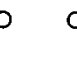

$\circ$

$\begin{array}{lll}0 & 0 & 0 \\ 0 & 0 & 0\end{array}$

QVT

Cosmop

$\star$

-

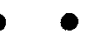

o

$\circ$

$\circ 0$

0

o

$\circ$

NZ, SE As

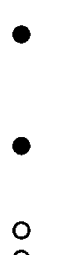




\begin{tabular}{|c|c|c|c|c|c|c|c|c|c|c|c|c|c|c|c|c|}
\hline \multirow[b]{2}{*}{ 61. LEMBOPHYLLACEAE (cont'd) } & \multirow{2}{*}{$\underset{\mathbf{I}}{\mathbf{H}}$} & \multicolumn{3}{|c|}{ C } & \multicolumn{3}{|c|}{$\mathbf{T}$} & \multicolumn{3}{|c|}{ WS } & \multicolumn{2}{|c|}{ WP } & \multicolumn{2}{|c|}{ FWP } & \multirow{2}{*}{$\begin{array}{c}\text { EXTRA } \\
\text { NSW }\end{array}$} & \multirow{2}{*}{$\begin{array}{c}\text { EXTRA } \\
\text { AUST }\end{array}$} \\
\hline & & $\mathbf{N}$ & C & $\mathbf{S}$ & $\mathbf{N}$ & C & $\mathbf{S}$ & $\mathbf{N}$ & $\mathbf{C}$ & $\mathbf{S}$ & $\mathbf{N}$ & $\mathbf{S}$ & $\mathbf{N}$ & $\mathbf{S}$ & & \\
\hline
\end{tabular}

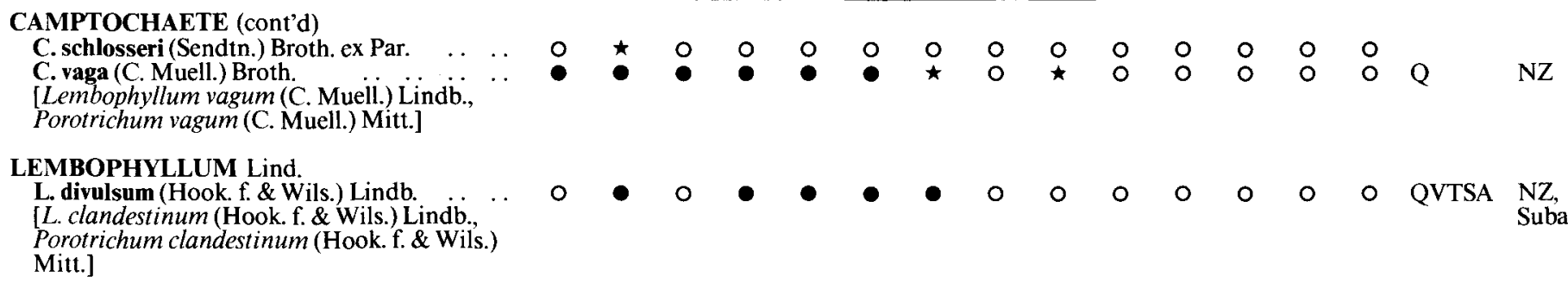

LEMBOPHYLLUM Lind

L. divulsum (Hook. f. \& Wils.) Lindb.

[L. clandestinum (Hook. f. \& Wils.) Lindib.,

Porotrichum clandestinum (Hook. f. \& Wils.)

Mitt.]

62. ECHINODIACEAE

\section{ECHINODIUM Jur.}

E. hispidum (Hook. f. \& Wils.) Reichdt.

[Sciaromium hispidum (Hook. f. \& Wils.)

Par., E. arboreum Broth.

E. parvulum Broth. \& Watts

$-0$

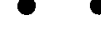

\section{HOOKERIACEAE}

ACHROPHYLLUM Vitt \& Crosby

A. dentatum (Hook. f. \& Wils.) Vitt \& Crosby

[Pterygophyllum dentatum (Hook. f. \& Wils.)

Dix., P. hepaticaefolium (Hampe \& C. Muell.)

Jaeg., $P$. nigellum (Hook. f. \& Wils.) Mitt.]

A. wattsii (Broth.) Vitt \& Crosby

[Pterygophyllum wattsii Broth.]
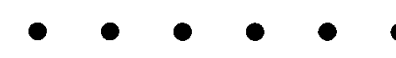

o

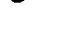

O

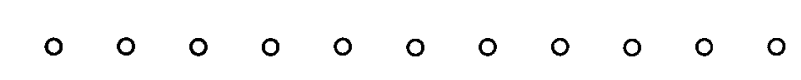




\section{CALYPTROCHAETA Desv}

C. apiculata (Hook. f. \& Wils.) Vitt
[Eriopus apiculatus (Hook. f. \& Wils.) Mitt.]

$\star 0$

00

$\circ$

0

00

o 0 VTS

NZ,

S Amer,

Subant I

\section{CYCLODICTYON Mitt.}

C. lepidum (Mitt.) Broth. \& Watts

[Hookeria karsteniana Broth. \& Geh.]

o

- 0

o

00

O 0

0

○

0

DISTICHOPHYLLUM Dozy \& Molk.

D. baileyanum $C$. Muell.

$\begin{array}{llllllllllllllll}0 & 0 & 0 & 0 & 0 & 0 & 0 & 0 & 0 & 0 & 0 & 0 & 0 & 0 & Q & \\ 0 & 0 & 0 & 0 & 0 & 0 & \star & 0 & 0 & 0 & 0 & 0 & 0 & 0 & V & \\ 0 & \star & 0 & 0 & 0 & 0 & 0 & 0 & 0 & 0 & 0 & 0 & 0 & 0 & V T & N Z\end{array}$

D. complanatum (Hampe) Mitt.

D. crispulum (Hook. f. \& Wils.) Mitt.

[Mniadelphus crispulus (Hook. f. \& Wils.) C.

Muell., M. adnatus (Hook. f. \& Wils.)

Reichdt.]

D. longicuspis Broth.

[D. leucoloma Broth. nom. nud.]

D. microcarpum (Hedw.) Mitt.

D. minutifolium C. Muell

D. pulchellum (Hampe) Mitt.

D. amblyophyllum (Hook. f. \& Wils)

Mniadelphus amblyophyllus (H Wils.) Mitt.

Wils.) Jaeg.]

D. rotundifolium (Hook. f. \& Wils.) C. Muell.

[D. squarrosulum $\mathrm{C}$. Muell.,
D. fissidentoides C. Muell.]

D. subminutifolium (Broth. \& Geh.) Fleisch.

[Mniadelphus subminutifolius Broth. \& Geh.]

D. whiteleggeanum C. Muell.

SAULOMA (Hook. f. \& Wils.) Mitt.

S. tenella (Hook. f. \& Wils.) Mitt. 


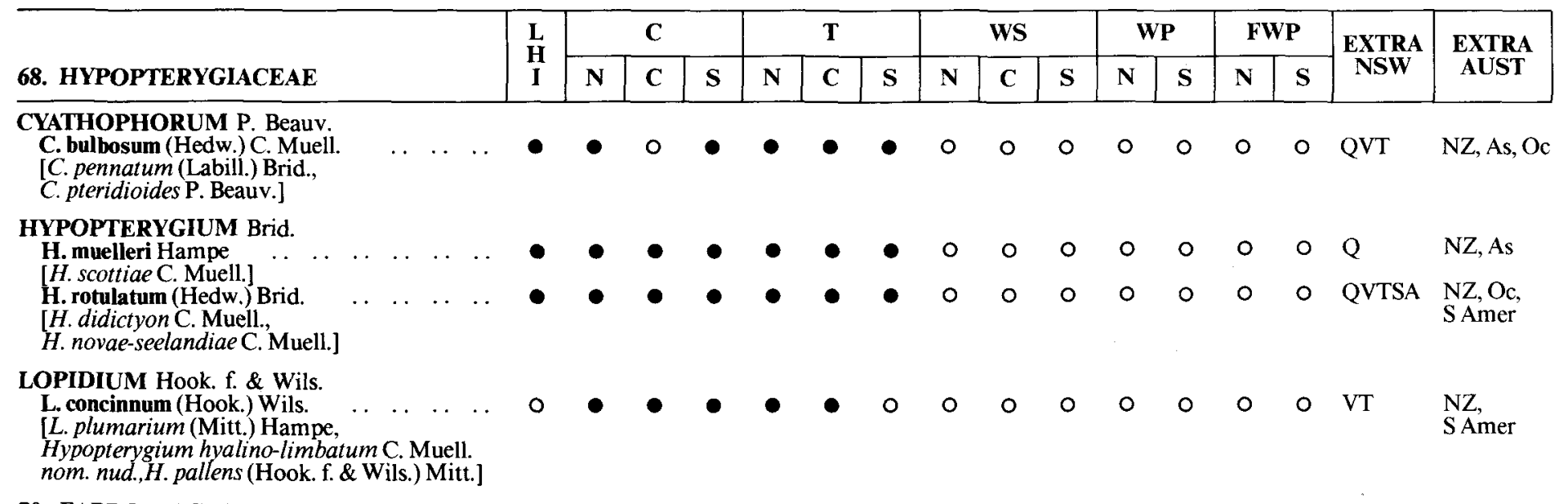

\section{FABRONIACEAE}

ANACAMPTODON Brid.
A. wattsii Broth. . . . .

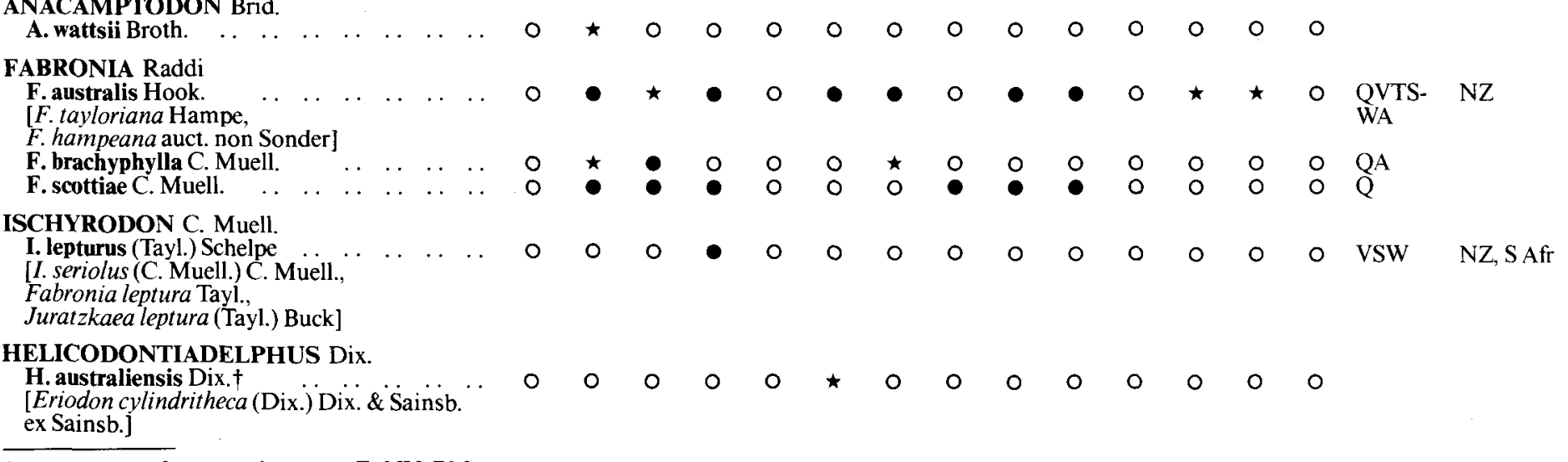

\footnotetext{
$\dagger$ Known only from specimens at E, NY, BM.
} 


\section{LESKEACEAE}

\section{PSEUDOLESKEA B.S.G}

P. imbricata (Hook. f. \& Wils.) Broth.

$[P$. calochlora Broth. \& Watts

Pseudoleskeopsis imbricata (Hook. f. \& Wils.)

Ther.]

\section{THUIDIACEAE}

ANOMODON Hook. \& Tayl.

A. tasmanicus Broth.

HAPLOHYMENIUM Dozy \& Molk.

H. pseudotriste (C. Muell.) Broth.

[H. brevinerve (Broth.) Broth.]

THUIDIUM B.S.G.

T. cymbifolium (Dozy \& Molk.) Dozy \& Molk.

[T. ramentosum (Mitt.) Mitt.

T. furfurosum (Hook. f. \& Wils.) Reichdt

Thuidiopsis furfurosa (Hook. f. \& Wils.)

Fleisch., Thuidium amblystegioides C. Muell.

T. hastatum (Mitt.) Reichdt.,T. suberectum

(Hampe) Jaeg.,T. unguiculatum (Hook. f. \&

Wils.) Fleisch.]

T. furfurosum (Hook. f. \& Wils.) Reichdt. var. sparsum (Hook. f. \& Wils.) Sainsb. .. .

T. laeviusculum (Mitt.) Jaeg.

T. liliputanum Broth

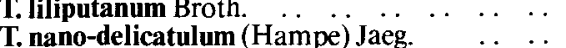

T. plumulosiforme (Hampe) Jaeg. .. $\quad \ldots \quad$.

T. protensulum C. Muell.

T. subglaucinum Card.

T. trachypodioides Broth. \& Watts 


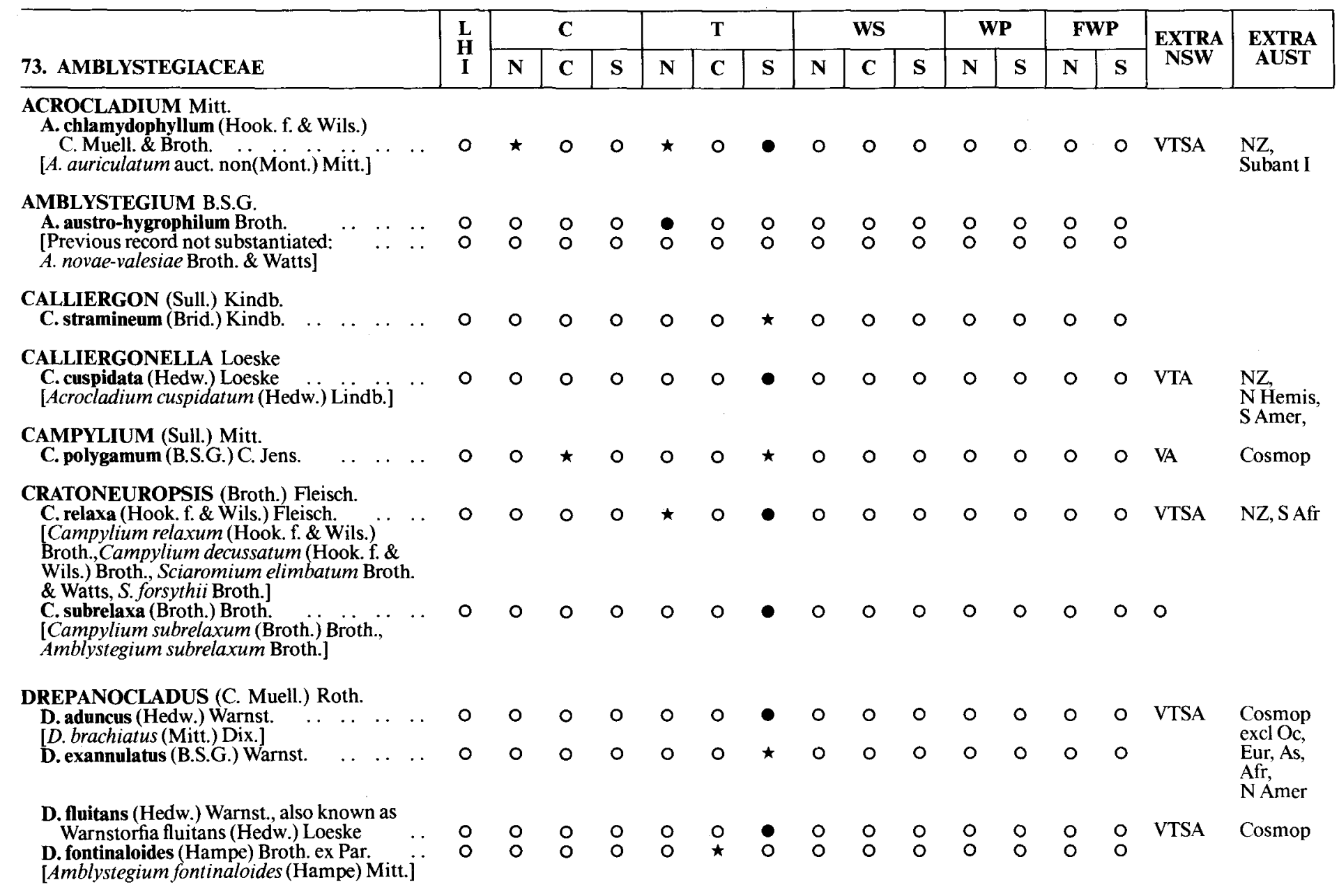

AMPYLIUM (Sull.) Mitt.
C. polygamum (B.S.G.) C. Jens.

RATONEUROPSIS (Broth.) Fleisch.

C. relaxa (Hook. f. \& Wils.) Fleisch.

Broth Campylium decussatum (Hook f

Wils.) Broth Sciaromium elimbatum Broth.

\& Watts, S. forsythii Broth.

C. subrelaxa (Broth.) Broth. ... ...

Campylium subrelaxum (Broth.) Broth.

DREPANOCLADUS (C. Muell.) Roth.

D. aduncus (Hedw.) Warnst

[D. brachiatus (Mitt.) Dix.]

D. fluitans (Hedw.) Warnst., also known as

Warnstorfia fluitans (Hedw.) Loeske
D. fontinaloides (Hampe) Broth. ex Par.

[Amblystegium fontinaloides (Hampe) Mitt.] 
D. uncinatus (Hedw.) Warnst., also known as

Sanionia uncinatus (Hedw.) Loeske

$\begin{array}{lllllll}. & 0 & 0 & 0 & 0 & 0 & 0 \\ . & 0 & 0 & 0 & 0 & 0 & 0\end{array}$

$\star$

\begin{tabular}{lllllllll}
$\circ$ & 0 & 0 & 0 & 0 & 0 & 0 & VTA & $\begin{array}{l}\text { Cosmop } \\
\text { Eur, As, } \\
\text { Amer }\end{array}$ \\
\hline
\end{tabular}

\section{LEPTODICTYUM (Schimp.) Warnst.}

L. riparium (Hedw.) Warnst.

[L. muelleri (Hampe \& C. Muell.) Broth.

Drepanocladus strictifolius Broth. \& Watts]

\section{BRACHYTHECIACEAE}

\section{BRACHYTHECIUM B.S.G}

B albicans (Hedw.

B. novae-valesiae Geh.

[Known only from type at $\dot{\mathrm{BM}}$ ]

B. paradoxum (Hook. f. \& Wils.) Jaeg. . . . . $\quad$ o

B. plumosum (Hedw.) B.S.G

[B. pseudo-plumosum (Brid.) Brockm.]

B. rivulare B.S.G.

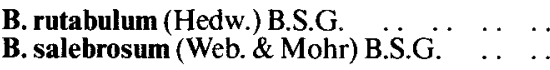

$$
\text { Brid.) Brockm.] }
$$

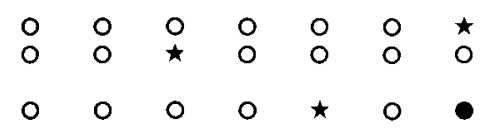

O

\section{EURHYNCHIUM B S G}

E. austrinum (Hook. f. \& Wils.) Jaeg

Platyhypnidium austrinum (Hook. f. \&

Wils.) Fleisch., Oxyrrhynchium austrinum

(Hook. f. \& Wils.) Broth.]

E. cucullatum (Mitt.) Stone \& Scott

[Rhynchostegiella cucullata (Mitt.) Dix.,

$R$. convolutifolia (Hampe) Broth.,

$R$. subconvolutifolia Broth. \& Watts]

E. muriculatum (Hook. f. \& Wils.) Jaeg

[Rhynchostegiella muriculata (Hook. f. \&

Wils.) Broth.]

E. speciosum (Brid.) Jur. ¿ (Brid) Warnst.

[Previous record not substantiated:

E. asperipes (Mitt.) Dix.]

0

O

0

$\circ$

$\begin{array}{ll}0 & 0 \\ 0 & 0\end{array}$

VTA

N Hemis

NZ, S Afr,

$S$ Amer

Cosmop

N Hemis,

S Afr

Cosmop

Cosmop

NZ,

Subant I 


\begin{tabular}{|c|c|c|c|c|c|c|c|c|c|c|c|c|c|c|c|c|}
\hline \multirow[b]{2}{*}{ 74. BRACHYTHECIACEAE (cont'd) } & \multirow{2}{*}{$\begin{array}{c}\mathbf{L} \\
\mathbf{H} \\
\mathbf{I}\end{array}$} & \multicolumn{3}{|c|}{ C } & \multicolumn{3}{|c|}{$\mathbf{T}$} & \multicolumn{3}{|c|}{ WS } & \multicolumn{2}{|c|}{ WP } & \multicolumn{2}{|c|}{ FWP } & \multirow{2}{*}{$\underset{\text { NSW }}{\text { EXTRA }}$} & \multirow{2}{*}{$\begin{array}{c}\text { EXTRA } \\
\text { AUST }\end{array}$} \\
\hline & & $\mathbf{N}$ & C & $\mathbf{S}$ & $\mathbf{N}$ & $\mathrm{C}$ & $\mathbf{S}$ & $\mathbf{N}$ & $\mathrm{C}$ & $\mathbf{S}$ & $\mathbf{N}$ & $\mathbf{S}$ & $\mathbf{N}$ & $\mathbf{S}$ & & \\
\hline
\end{tabular}

OXYRRHYNCHIUM (B.S.G.) Warnst.

O. howeanum Broth. \& Watts .. ..

RHYNCOSTEGIELLA (B.S.G.) Limpr.

R. campylioides Broth. \& Watts .. . . .

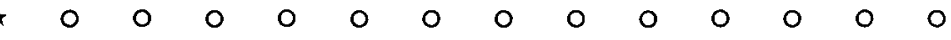

RHYNCHOSTEGIUM B.S.G.

R. sp. cf. dentiferum (Hampe) Jaeg.

R. laxatum (Mitt.) Par.

R. patulum Jaeg.

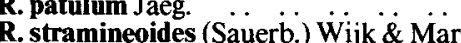

R.

R. pseudo-stramineum Mitt. nom. illeg.

R. tenuifolium (Hedw.) Reichdt.

[R. collatum (Hook. f. \& Wils.) Broth. \&

Watts, R. subclavatum (Hampe) Jaeg.]

R. tenuifolium var. howeanum Broth. \& Watts

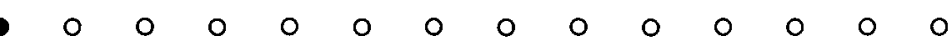

\section{ENTODONTACEAE}

ENTODON C. Muell.

E. mackayensis C. Muell.

E. pancherianus (Besch.) Jaeg.

\begin{tabular}{llllllllllllllll}
0 & 0 & 0 & 0 & 0 & $\star$ & 0 & 0 & 0 & 0 & 0 & 0 & 0 & 0 & VA & \\
0 & 0 & 0 & 0 & 0 & $\bullet$ & $\star$ & 0 & 0 & 0 & 0 & 0 & 0 & 0 & VTA & NZ \\
0 & $\star$ & 0 & 0 & 0 & 0 & 0 & $\star$ & $\star$ & 0 & 0 & 0 & 0 & 0 & QV & \\
0 & 0 & 0 & 0 & 0 & $\star$ & 0 & 0 & 0 & 0 & 0 & 0 & 0 & 0 & & \\
$\bullet$ & 0 & $\bullet$ & $\bullet$ & $\star$ & $\bullet$ & $\bullet$ & 0 & $\bullet$ & $\star$ & 0 & 0 & 0 & 0 & QVTS- & NZ, As, \\
\hline & $\bullet$ & & & & & & & & & & & & & WA & S Amer
\end{tabular}

PIAGIOTHECIACEAE

\section{PLAGIOTHECIUM B.S.G.}

P. denticulatum (Hedw.) B.S.G.

P. novae-valesiae Broth.

-

o

o

0

$\begin{array}{llllll}0 & 0 & 0 & 0 & 0\end{array}$

\section{SEMATOPHYLLACEAE}

MEIOTHECIUM Mitt.
M. wattsii (Broth.) Broth.

M. wattsii (Broth.) Broth.
[Pterogoniella wattsii Broth.]

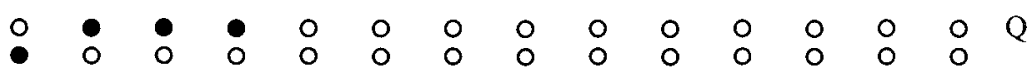

Oc 
PUNGENTELLA C. Muell

P. subfalcatulum (Broth. \& Watts) C. Muell.

Rhaphidorrhynchium subfalcatulum (Broth

\& Watts) Broth., Rhaphidostegium

subfalcatulum Broth. \& Watts]

\section{SEMATOPHYLLUM Mitt.}

S. aciculum (Dix.) Dix. Rhaphidostegium aciculum Broth. ex Dix.]

[Rhaphidostegium aciculum Broth. ex Dix.]

S. amoenum (Hedw.) Mitt

[Rhaphidorrhynchium amoenum (Hedw.)

Fleisch., Rhaphidostegium callidioides (C.

Muell.) Jaeg., Rhaphidorrhynchium

callidioides (C. Muell.) Broth.]

S. contiguum (Mitt) Mitt.

Shaphidostegium contiguum (Mitt) $\ddot{P a r}$..

[Rhaphidostegium contiguum (Mitt.) Par.]

Broth.

[Rhaphidostegium crassiusculum Brid.]

S. elachistos (Besch.) Broth.

S. homomallum (Hampe) Broth. $\quad \ldots \quad \cdots \quad$.

[Rhaphidorrhynchium homomallum

(Hampe) Mitt.,Rhaphidostegium

homomallum (Hampe) Broth.]

S. jolliffii (Hook. f.) Dix.

Rhaphidorrhynchium joll

S. luciduloides (Dix.) Mitt. . $\ddot{\text {. }}$

[Rhaphidostegium luciduloides Dix.]

S. uncinatum Stone \& Scott

Rhaphidorrhynchium tenuirostre (广்ok $)$

Broth., Rhaphidostegium tenuirostre (Hook.)

Jaeg.]

S. wattsii (Par.) Broth.

Rhaphidostegium wattsii $\mathrm{Par}$

Rhaphidostegium micropyxis Broth.]

TAXITHELIUM Spruce ex Mitt.

T. novae-valesiae (Broth.) Broth.

[Isopterygium novae-valesiae Broth.]

TRICHOSTELEUM Mitt.

T. muscicolum Broth. . .

WARBURGIELLA $C$ MUell.

W. subleptorrhynchoides (Fleisch.) Fleisch.

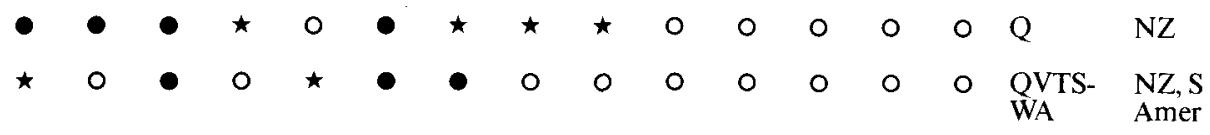

\begin{tabular}{|c|c|c|c|c|c|c|c|c|c|c|c|c|c|c|c|}
\hline$\star$ & $\star$ & 0 & 0 & 0 & $\star$ & - & 0 & 0 & 0 & 0 & 0 & 0 & 0 & QVTSA & $\begin{array}{l}\text { NZ, S Afr, } \\
\text { S Amer }\end{array}$ \\
\hline 0 & 0 & 0 & 0 & 0 & $\star$ & 0 & O & 0 & $\mathrm{O}$ & 0 & 0 & 0 & 0 & & $\begin{array}{l}\text { SAmer } \\
\text { NZ, Afr, } \\
\text { Amer }\end{array}$ \\
\hline $\begin{array}{l}0 \\
0\end{array}$ & $\begin{array}{l}0 \\
0\end{array}$ & $\stackrel{\bullet}{\star}$ & 8 & $\begin{array}{l}0 \\
0\end{array}$ & $\begin{array}{l}0 \\
\star\end{array}$ & $\stackrel{\star}{\star}$ & $\begin{array}{l}0 \\
0\end{array}$ & $\begin{array}{l}0 \\
0\end{array}$ & $\begin{array}{l}0 \\
0\end{array}$ & $\begin{array}{l}0 \\
0\end{array}$ & $\begin{array}{l}0 \\
0\end{array}$ & $\begin{array}{l}0 \\
0\end{array}$ & $\begin{array}{l}0 \\
0\end{array}$ & QVTS- & $\begin{array}{l}\mathrm{Oc} \\
\mathrm{NZ}, \mathrm{As}, \mathrm{Oc}\end{array}$ \\
\hline
\end{tabular}




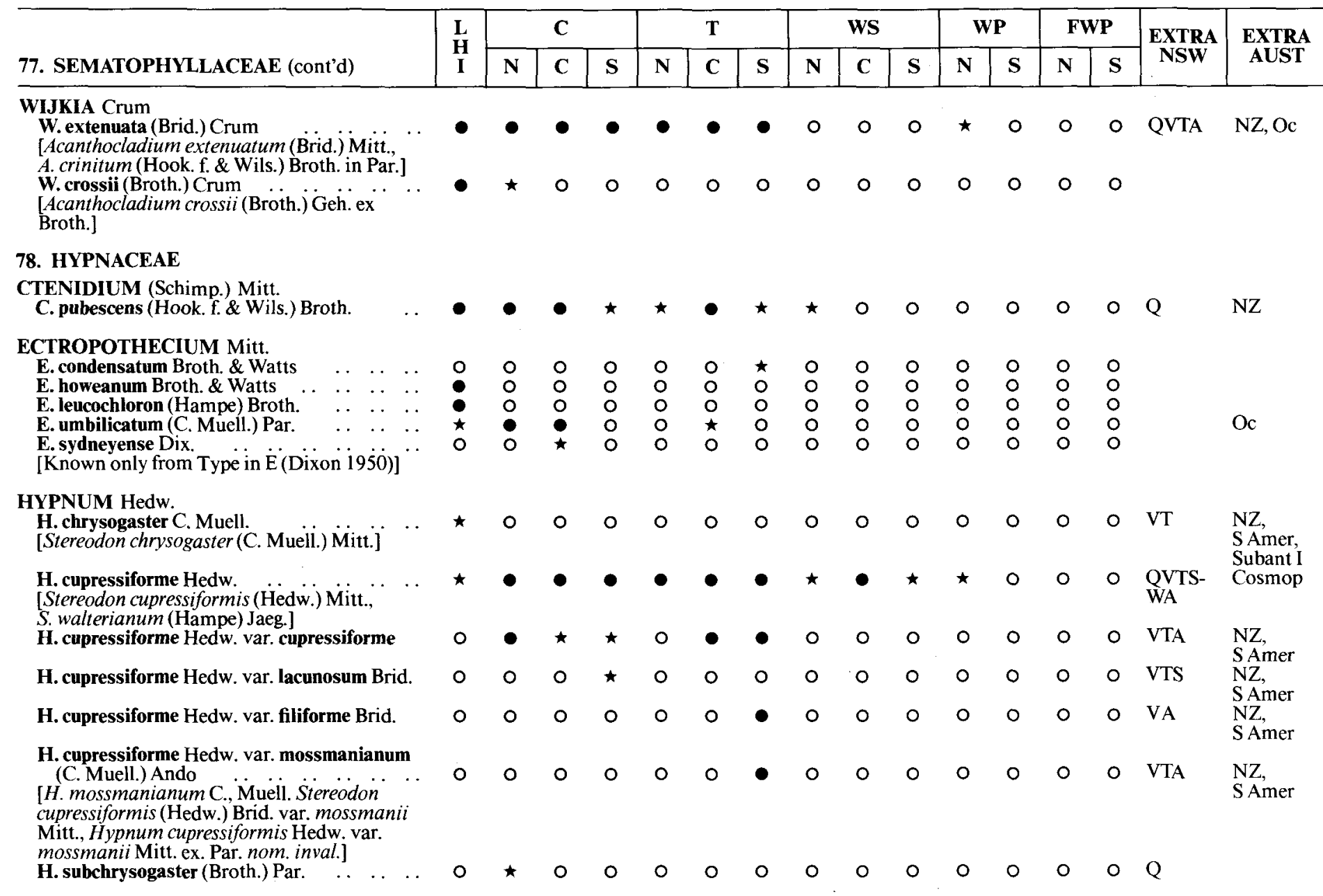


ISOPTERYGIUM Mitt.

I. candidum (C. Muell.) Jaeg.

[I. candidum (C. Muelliculum (Sull.) Mitt.]

I. amblyocarpum (Hampe) Broth.

l. amoenum Broth

I. arachnoideum Broth.

I. howeanum Broth. \& Watts

I. latifolium Broth.

I. minutirameum (C. Muell.) Jaeg.

I l austro-pusillum (C. Muell.) Jaeg.

?. austro-subulatum C. Muell. nom. nud.]

I. pseudo-subulatum (C. Muell.) Par.

I. subarachnoideum Broth

$\begin{array}{llllllllllllll}0 & 0 & 0 & 0 & 0 & 0 & \star & 0 & 0 & 0 & 0 & 0 & 0 & 0 \\ 0 & \star & 0 & 0 & 0 & 0 & 0 & 0 & 0 & 0 & 0 & 0 & 0 & 0 \\ 0 & 0 & 0 & 0 & 0 & 0 & 0 & 0 & 0 & 0 & 0 & 0 & 0 & 0 \\ 0 & 0 & 0 & 0 & 0 & 0 & 0 & 0 & 0 & 0 & 0 & 0 & 0 & 0 \\ 0 & \star & 0 & 0 & 0 & 0 & 0 & 0 & 0 & 0 & 0 & 0 & 0 & 0 \\ 0 & 0 & 0 & 0 & 0 & 0 & 0 & 0 & 0 & 0 & 0 & 0 & 0 & 0\end{array}$

Q QV Oc, NZ,

VESICULARIA (C. Muell.) C. Muell.

V montagnei (Bel.) Broth

V. reticulata (Dozy \& Molk.) Besch.

V. rivalis Broth

V. slateri (Hampe) Broth

[Ectropothecium slateri (Hampe) Jaeg.]

\section{BUXBAUMIACEAE}

\section{BUXBAUMIA Hedw.}

B. colyerae Burges

$\begin{array}{lllllll}\ldots & \ldots & \ldots & \bullet & 0 & 0 & 0 \\ & \ldots & \ldots & 0 & 0 & ? & 0 \\ \ldots & \ldots & \ldots & 0 & \star & 0 & 0 \\ \ldots & \ldots & \ldots & 0 & \bullet & 0 & 0\end{array}$

$\begin{array}{lll}0 & 0 & 0 \\ 0 & 0 & 0\end{array}$

As, Oc $\begin{array}{llllllllllllll}0 & \star & \star & 0 & 0 & 0 & 0 & 0 & 0 & 0 & 0 & 0 & 0 & 0 \\ 0 & 0 & 0 & 0 & 0 & 0 & 0 & 0 & 0 & 0 & 0 & 0 & 0 & 0\end{array}$

83. POLYTRICHACEAE

ATRICHUM P. Beauv.

A. androgynum (C. Muell.) Jaeg. var.

androgynum

A. angustatum var. polysetum $\ddot{\text { Watts }} \ddot{*}$

Whitel. nom. nud., A. ligulatum Mitt.,

Catharinea leptocylindrica C. Muell, $C$.

muelleri C. Muell. \& Hampe]

POGONATUM P. Beauv.

P. brachypodium (C. Muell.) Watts \& Whitel.

P. canarae (C. Muell.) Par.

P. subulatum (Brid.) Brid.

[P. australasicum (C. Muell. \& Hampe) Jaeg.]

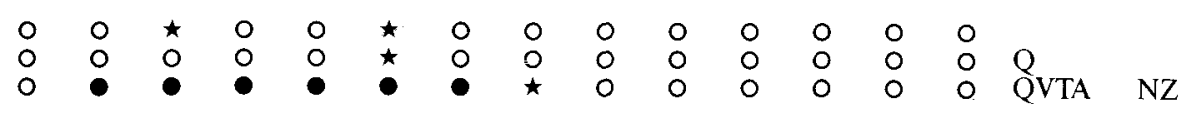




\begin{tabular}{|c|c|c|c|c|c|c|c|c|c|c|c|c|c|c|c|c|c|c|}
\hline \multirow[b]{2}{*}{ 83. POLYTRICHACEAE (cont'd) } & & & \multirow{2}{*}{$\underset{\mathbf{I}}{\mathbf{H}}$} & \multicolumn{3}{|c|}{ C } & \multicolumn{3}{|c|}{$\mathbf{T}$} & \multicolumn{3}{|c|}{ WS } & \multicolumn{2}{|c|}{$\mathbf{W P}$} & \multicolumn{2}{|c|}{ FWP } & \multirow{2}{*}{$\underset{\text { NSW }}{\text { EXTRA }}$} & \multirow{2}{*}{$\begin{array}{c}\text { EXTRA } \\
\text { AUST }\end{array}$} \\
\hline & & & & $\mathbf{N}$ & C & $\mathbf{S}$ & $\mathbf{N}$ & C & $\mathbf{S}$ & $\mathbf{N}$ & C & $\mathbf{S}$ & $\mathbf{N}$ & $\mathbf{S}$ & $\mathbf{N}$ & $\mathbf{S}$ & & \\
\hline $\begin{array}{l}\text { POLYTRICHADELPHUS (C. Muell.) } \\
\text { P. magellanicus (Hedw.) Mitt. } \\
{[P \text {. innovans (C. Muell.) Jaeg. }]}\end{array}$ & $\begin{array}{c}\text { Mit } \\
\cdots\end{array}$ & & 0 & 0 & 0 & $\star$ & 0 & 0 & $\star$ & 0 & 0 & 0 & 0 & 0 & 0 & 0 & QVT & $\begin{array}{l}\mathrm{NZ}, \mathrm{S} \\
\text { Amer }\end{array}$ \\
\hline $\begin{array}{l}\text { POLYTRICHASTRUM G.L. Smith } \\
\text { P. alpinum (Hedw.) G.L.Smith } \\
\text { [Polytrichum alpinum Hedw.] }\end{array}$ & $\cdots$ & . & 0 & 0 & 0 & 0 & 0 & 0 & $\star$ & 0 & 0 & 0 & 0 & 0 & 0 & 0 & VTA & $\begin{array}{l}\text { NZ, } \\
\text { Cosmop }\end{array}$ \\
\hline 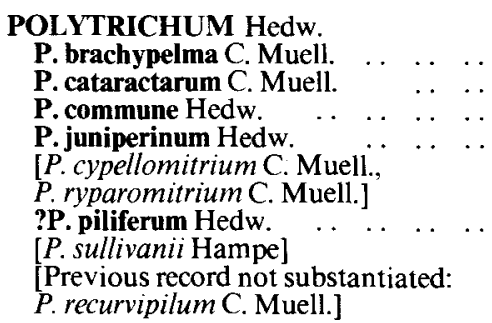 & $\begin{array}{l}\cdots \\
\ldots \\
\cdots \\
\ldots \\
\ldots\end{array}$ & $\begin{array}{l}\cdots \\
\cdots \\
\cdots \\
\cdots \\
\\
\\
\end{array}$ & $\begin{array}{l}0 \\
0 \\
0 \\
0\end{array}$ & $\begin{array}{l}0 \\
0 \\
0 \\
0\end{array}$ & $\begin{array}{l}\star \\
0 \\
0\end{array}$ & $\begin{array}{l}0 \\
\star \\
0\end{array}$ & $\begin{array}{l}0 \\
0 \\
\vdots \\
\star\end{array}$ & $\begin{array}{l}0 \\
0 \\
0\end{array}$ & $\begin{array}{l}0 \\
0 \\
0\end{array}$ & $\begin{array}{l}0 \\
0 \\
0 \\
\star\end{array}$ & $\begin{array}{l}0 \\
0 \\
0 \\
0\end{array}$ & $\begin{array}{l}0 \\
0 \\
0 \\
0\end{array}$ & $\begin{array}{l}0 \\
0 \\
0 \\
0\end{array}$ & $\begin{array}{l}0 \\
0 \\
0 \\
0\end{array}$ & $\begin{array}{l}0 \\
0 \\
0 \\
0\end{array}$ & $\begin{array}{l}0 \\
0 \\
0 \\
0\end{array}$ & $\begin{array}{l}\text { QVTA } \\
\text { QVTSA }\end{array}$ & $\begin{array}{l}\text { Cosmop } \\
\text { Cosmop }\end{array}$ \\
\hline $\begin{array}{l}\text { PSILOPILUM Brid. } \\
\text { P. australe Hook. f. \& Wils.) Mitt. }\end{array}$ & . & $\cdots$ & 0 & 0 & 0 & 0 & 0 & 0 & - & 0 & 0 & 0 & $\mathrm{O}$ & 0 & 0 & o & VT & $\begin{array}{l}\text { NZ, S Afr, } \\
\text { Subant I }\end{array}$ \\
\hline $\begin{array}{l}\text { P. crispulum (Hook. f. \& Wils.) Mitt. } \\
{[P . \text { pyriforme (Hampe) Jaeg. }]}\end{array}$ & . & . & 0 & 0 & 0 & 0 & 0 & $\star$ & $\bullet$ & 0 & 0 & 0 & 0 & 0 & 0 & 0 & VT & $\mathrm{NZ}$ \\
\hline
\end{tabular}

\section{DAWSONIACEAE}

\section{DAWSONIA $\mathrm{R}$. Br.}

D. longiseta Hampe $\cdots$. $\ldots$ victoriae $\mathrm{C}$. Mueli.]

D. polytrichoides $\mathrm{R}$. $\mathrm{Br}$.

D. superba Grev. var. pulchra ( $\left.\dot{W}_{\mathrm{ij} k}\right) \ddot{Z}_{\mathrm{Z} a n t}$

[S. pulchra Wijk, D. intermedia C. Muell. ex

Schlieph. \& Geh., D. longifolia (Bruch. \&

Schimp. in B.S.G.) Zant. var. superba Zant.1

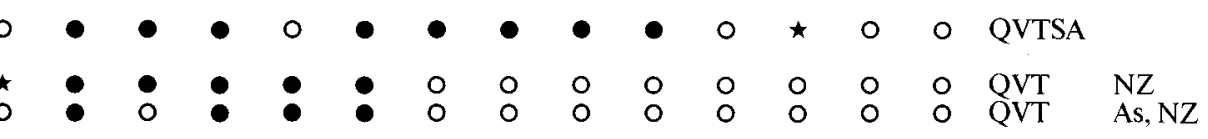




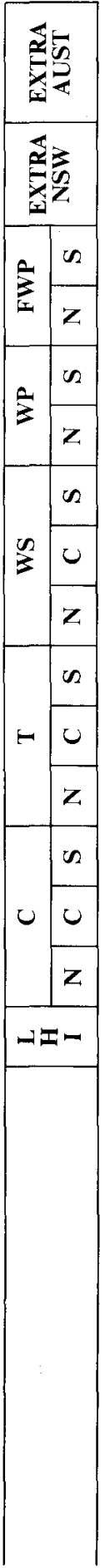




\begin{tabular}{|c|c|c|c|c|c|c|c|c|c|c|c|c|c|c|c|}
\hline $\mathbf{L}$ & \multicolumn{3}{|c|}{ C } & \multicolumn{4}{|c|}{ T } & \multicolumn{4}{|c|}{ WS } & \multicolumn{3}{|c|}{ WP } & \multicolumn{2}{|c|}{ FWP } & EXTRA & EXTRA \\
\cline { 2 - 13 } & $\mathbf{N}$ & $\mathbf{C}$ & $\mathbf{S}$ & $\mathbf{N}$ & $\mathbf{C}$ & $\mathbf{S}$ & $\mathbf{N}$ & $\mathbf{C}$ & $\mathbf{S}$ & $\mathbf{N}$ & $\mathbf{S}$ & $\mathbf{N}$ & $\mathbf{S}$ & NSW & AUST \\
\hline
\end{tabular}




\section{INDEX TO SYNONYMS}

Acanthocladium crinitum (Hook. f. \& Wils.) Broth. in Par. = Wijkia extenuata

A. crossii (Broth.) Geh. ex Broth. = Wijkia crossi

A. extenuatum (Brid.) Mitt. = Wijkia extenuata

Acaulon austro-muticum Geh. ex Roth $=\mathrm{A}$. integrifolium

A. sullivanii $\mathrm{C}$. Muell. $=\mathrm{A}$. integrifolium

Acrocladium auriculatum auct. non (Mont.) Mitt. = A. chlamydophyllum

A. cuspidatum (Hedw.) Lindb. = Calliergonella cuspidata

Aloina ambigua (B.S.G.) Limpr. = A. aloides var, ambigua

Amblystegium subrelaxum Broth. = Cratoneuropsis subrelaxa

A. fontinaloides (Hampe) Mitt. $=$ Drepanocladus fontinaloides

Andreaea amblyophylla C. Muell. ex Broth. = A. mutabilis

A. rupestris auct. non Hedw. $=$ A. mutabilis

A subulatissima C. Muell = A. subulata

Anisothecium pycnoglossum Broth. = Dicranella pycnoglossa

A. jamesonii Mitt. = Dicranella jamesonii

Aongstroemia stackhousiana C. Muell. = Dicranella stackhousiana

A. rufo-aureum (Hampe) Willis = Ditrichum rufo-aureum

Aplodium lineare Mitt. $=$ Orthodontium lineare

Archidium brisbanicum Broth. = Eccremidium brisbanicum

A. stolonaceum C. Muell. = Eccremidium pulchellum

Astomum brisbanicum (C. Muell.) Broth. = Trachycarpidium brisbanicum

A. cylindricum (Tayl.) Mitt. = Tetrapterum cylindricum

A. novae-valesiae Broth. ex Roth = Weissia novae-valesiae

A. viride C. Muell. = Pleuridium viride

A wattsi Broth. ex Roth $=$ Trachycarpidium brisbanicum

Atrichum angustatum (Brid.) B.S.G. var. polysetum Watts \& Whitel. = A.

$$
\text { androgynum }
$$

A. Ligulatum Mitt. $=$ A. androgynum

Barbella trichophoroides (C. Muell.) Broth. = B. enervis

Barbula atro-virens $(\mathrm{Sm}$.) Schimp. = Desmatodon convolutus

B. calycina Schwaegr. = Tortella calycina

$B$. pilifera (Hook.) Brid. = B. crinita

B. pseudopilifera C. Muell. \& Hampe $=$ B. crinita

$B$. rotundata Lindb. \& Arn. = Bryoerythrophyllum rotundatum

$B$. subspiralis Hampe $=B$. hornschuchiana

Bartramia erecta (Hampe) Broth. = B. hampei

$B$. mossmanniana C. Muell. = B. halleriana

B. strictifolia Tayl. $=$ B. stricta

Bartramidula hampei Mitt. = Bartramia hampei
Bescherellia cyrtopus $\mathrm{F}$. Muell. $=\mathrm{B}$. brevifolia

Blindia tenuifolia (Hook.f. \& Wils.) Mitt. = B. robusta

Brachymenium chloroblastum Watts \& Whitel. = B. preissianum

$B$. pilosithecium Watts \& Whitel. = B. preissianum

Brachysteleum australe (Hampe) Jaeg. = Ptychomitrium australe B. laxifolium C. Muell. = Ptychomitrium laxifolium Brachythecium pseudo-plumosum (Brid.) Brockm. = B. plumosum Breutelia commutata Jaeg. = B. affinis

$B$. divaricata $($ Mitt.) Mitt. = B. pendula

B. fusco-aurea Broth. = B. pendula

B. sieberi Hornsch. ex C. Muell. = B. pendula

Bruchia amoena $C$. Muell. = Trematodon amoenum

$B$. whiteleggei $\mathrm{C}$. Muell. $=$ Eccremidium minutum

Bryobartramia robbinsii Sainsb. = B. novae-valesiae

Bryoerythrophyllum binsil (R. Br. ter.) Wijk \& Marg. = B. jamesonii

Bryum abruptinervium $\mathrm{C}$. Muell. = B. billardieri var. billardieri

B. aequicollum Broth. \& Watts $=$ B. dichotomum

B. affine Lindb. \& Arn. = B. creberrimum

B. amoenum Wright non Broth. = B. subfasciculatum

$B$. appressifolium Broth. $=\mathrm{B}$. australe

$B$. austro-affine Broth. = B. pseudotriquetrum

$B$. austro-alpinum C. Muell. = B. crassum

B. bailevi Broth. $=$ B. nitens

B. bicolor Dicks. $=$ B. pachytheca

B. bimum (Brid.) Turn. = B. pseudotriquetrum

$B$. brachyaris $\mathrm{C}$. Muell. in Mitt. $=\mathrm{B}$. billardieri var. billardieri

B. brachytheciella C. Muell. = B. dichotomum

B. breviramulosum (Hampe) Mitt. = B. billardieri var. billardieri

B. brunneidens C. Muell. = B. billardieri var. billardieri

$B$. cheelii Broth. $=$ B. muehlenbeckii

B. clavatum Hook. f. \& Wils. = B. erythrocarpoides

B. crenatidens $\mathrm{C}$. Muell. = B. billardieri var. billardieri

B. crispatum (Hampe) Mitt. = B. billardieri var. billardieri

B. curvicollum Mitt. = B. erythrocarpoides

$B$. dilatato-marginatum $\mathrm{C}$. Muell. = B. subfasciculatum

$B$. diversinerve Broth. \& Watts = B. erythrocarpoides

B. ervthropyxis C. Muell. = B. capillare ssp. torquescens

B. filarium Broth, = B. erythrocarpoides

B. forsythii Broth. = B. billardieri var. billardieri

B. gambierense C. Muell. = B. pachytheca

$B$. globulare Hampe in C. Muell. = B. billiardieri var. billardieri

$B$. howeanum Broth \& Watts = B. campylothecium

B. immarginatum Broth. = B. capillare

B. kiamae Broth. = B. erythrocarpoide

$B$. laxirete Broth $=$ B caespiticium 
B. leptopelma C. Muell. = B. chrysoneuron

B. leptothecioides Broth. \& Watts = B. capillare

B. leptothecium Tayl. $=$ B. billardieri var. billardieri

B. lochoneuron $\mathrm{C}$. Muell. = B. chrysoneuron

B. microerythrocarpum C. Muell. \& Kindb. = B. pachytheca

B. micropachypoma Broth. = B. nitens

B. microthecium $\mathrm{C}$. Muell. = B. chrysoneuron

B. oblongifolium C. Muell. = B. blandum

B. olivaceum (Hampe) Mitt. = B. billardieri var. billardieri

B. pachypoma Mont. = B. plumosum

B. pachypomatulum Broth $=$ B nitens

B. peraristatum C. Muell. = B. campylothecium

B. philonotideum Broth. \& Watts = B. dichotomum

B. pimpanae C. Muell. = B. dichotomum

B. pohliaeopsis $\mathrm{C}$. Muell. = B. billardieri var. billardieri

B. pusillum Broth. = B. capillare

B. pyrothecium C. Muell. \& Hampe = B. capillare ssp. torquescens

B. robustum Hampe $=$ B. billardieri var. billardieri

B. rufescens Hook. f. \& Wils. = B. billardieri var. billardieri

B. subaeneum Hampe \& C. Muell. = B. pachytheca

B. subatro-purpureum C. Muell. = B. coronatum

B. subcrispatum C. Muell. = B. billardieri var. billardier

B. subcupulatum C. Muell. ex Rodw. = B. dichotomum

B. subcurvicollum Broth. = B. erythrocarpoides

B. suberythrocarpum C. Muell. = B. chrysoneuron

B. sublonginervium Geh. in Watts \& Whitel. = B. capillare

B. subolivaceum C. Muell. = B. subfasciculatum

$B$. subpachypoma Hampe $=$ B. nitens

B. subtomentosum (Hampe) Mitt. = B. billardieri var. billardieri

B. subventricosum Broth. = B. pseudotriquetrum

B. sullivanii C. Muell. = B. pachytheca

B. synoicum C. Muell. = B. capillare ssp. torquescens

B. tasmanicum Hampe $=$ B. creberrimum

$B$. viridulum C. Muell. $=$ B. subfasciculatum

$B$. wattsii Broth. $=$ B. chrysoneuron

$B$. whiteleggei Broth. $=$ B. capillare

Calomnion laetum Hook. f. \& Wils. = C. complanatum

Calymperes armatum Broth. = Syrrhopodon incompletus

Campylium relaxum (Hook. f. \& Wils.) Broth. = Cratoneuropsis relaxa

C. decussatum (Hook. f. \& Wils.) Broth. = Cratoneuropsis relaxa

C. subrelaxum (Broth.) Broth. = Cratoneuropsis subrelaxa

Camptochaete brisbanica C. Muell. = C. arbuscula var. deflexa

C. deflexa (Wils.) Jaeg. = C. arbuscula var. deflexa

C. ramulosa (Mitt.) Jaeg. $=$ C. arbuscula
Campylopus appressifolius Mitt. $=$ C. clavatus

C. institius Hook. f. \& Wils. = C. clavatus

C. novae-valesiae Broth. $=\mathrm{C}$. pallidus

C. pudicus (C. Muell.) Jaeg. = C. introflexus

C. tasmanicus Par. $=\mathrm{C}$. introflexus

C. torquatus Mitt. $=$ C. pallidus

C. woollsii (C. Muell.) Par. = C. pallidus

Catharinea leptocylindrica C.Muell.=Atrichum androgynum var.

androgynum

C. muelleri C.Muell.\&Hampe = Atrichum androgynum var. androgynum Ceratodon convolutus Reichdt. $=\mathrm{C}$. purpureus

C. stenocarpus Bruch. \& Schimp. ex C. Muell. = C. purpureus

Cheilothela chilensis (Mont.) Broth. = Chrysoblastella chilensis

Conostomum australe $\mathrm{Sw} .=\mathrm{C}$. pentastichum

Cryphaea muelleri (Hampe) Broth. = C dilatata

C. brevidens C. Muell. = C. tenella

C. novae-valesiae C. Muell. = Papillaria flexicaulis

C. squarrulos Hampe $=$ C. ovalifolia

Cryphidium muelleri (Hampe) Broth. = Cryphaea dilatata

C. ovalifolium (C. Muell.) Broth. = Cryphaea ovalifolia

C. squarrulosum (Hampe) Broth. = Cryphaea ovalifolia

Cvathophorum pennatum (Labill.) Brid. $=$ C. bulbosum

C. pteridioides P. Beauv. = C. bulbosum

Cyptodon dilatatus (Hook. f. \& Wils.) Par. \& Schimp. = Cryphaea dilatata

C. ovalifolius (C. Muell.) Fleisch. = Cryphaea ovalifolia

Cyrtopus bescherelloides $\mathrm{C}$. Muell. = Bescherellia brevifolia

Dawsonia appress $a$ Hampe $=\mathrm{D}$. longiseta

D. intermedia C.Muell. ex Schlieph. \& Geh. = D. superba var. pulchra

D. longifolia (Bruch. \& Schimp. in B.S.G.) Zant. var. superba Zant. =

D. superba var. pulchra

D. pulchra Wijk = D. superba var. pulchra

D. victoriae C. Muell. $=$ D. longiseta

Desmatodon adustus Mitt. = Barbula hornschuchiana

Dichodontium wattsii (Broth.) Broth. = Dicranella cardotii

Distichophyllum leucoloma Broth. = D. longicuspis

Dicnemoloma sieberianum (Hornsch.) Broth. = Dicnemoloma pallidum

Dicranella wattsii Broth. = D. cardotil

D. tricruris (C. Muell.) Mitt. = D. dietrichiae

Dicranoloma bartramioides (Broth.) Par. = D. menziesii

Dicranum dicarpum Nees = Dicranoloma dicarpum

D. sullivanii $\mathrm{C}$. Muell. = Dicranoloma sullivanil

D. whiteleggei $\mathrm{C}$. Muell. = Dicranoloma argutum

D. scoparium Hedw. ssp. bonjeani (De Not.) Grout = Dicranum bonjeanii 
Didymodon rotundatus (Lindb. \& Arn.) Par. = Bryoerthrophyllum rotundatum

Distichophyllum amblyophyllum (Hook. f. \& Wils.) Mitt. = D. pulchellum $D$. fissidentoides $\mathrm{C}$. Muell. $=\mathrm{D}$. rotundifolium

D. leucoloma Broth. = D. longicuspis

D. squarrosulum C. Muell. = D. rotundifolium

Ditrichum affine (C. Muell.) Hampe = D. difficile

D. baileyi $\mathrm{C}$. Muell. $=\mathrm{D}$. difficile

D. elongatum (Hook. f. \& Wils.) Mitt. = D. cylindricarpum

D. flaccidulum C. Muell. in Watts \& Whitel. = D difficile

D. flexifolium Hampe $=\mathrm{D}$. difficile

D. muelleri (Hampe) Hampe $=\mathrm{D}$. difficile

D. setosum (Hook. f. \& Wils.) Reichdt. = D. difficile

D. subbrachycarpum (C. Muell.) Par. = D. brachycarpum

Drepanocladus brachiatus (Mitt.) Dix. = D. aduncus

D. strictifolius Broth. \& Watts = Leptodictyum riparium

Echinodium arboreum Broth. $=$ E. hispidum

Ectropothecium slateri (Hampe) Jaeg. = Vesicularia slateri

Encalypta australis Mitt. = E. vulgaris

E. tasmanica Hampe $=$ E. vulgaris

Entosthodon apophysata (Tayl.) Mitt. = Funaria apophysata

E. aristatus (Broth.) Par. = Funaria aristata

E. gracilis Hook. f. \& Wils. = Funaria gracilis

E. smithurstii (Broth. \& Geh.) Par. = Funaria smithurstii

E. squarrifolius (Broth.) Par. = Funaria squarrifolia

Ephemerum whiteleggei Broth. \& Geh. = Eccremidium minutum

Eriopus apiculatus (Hook. f. \& Wils.) Mitt. = Calyptrochaeta apiculata

Euptychium cuspidatum (Mitt.) Mitt. = E. setigerum

Fabronia hampeana auct. non Sonder $=$ F. australis

F. leptura Tayl. = Ischyrodon lepturus

$F$. tavloriana $\mathrm{Hampe}=\mathrm{F}$. australis

Fissidens brevifolius Hook. f. \& Wils. = F. taylorii

$F$. dietrichiae $\mathrm{C}$. Muell. $=\mathrm{F}$. crassipes

F. elamellosus C. Muell. \& Hampe = F. taylorii

F. macrodus Hampe $=\mathrm{F}$. taylorii

$F$. muelleri (Hampe) Mitt. = F. fontanus

$F$. praemollis Broth. = F. crassipes

F semilimbatus $\mathrm{C}$. Muell. \& Hampe $=\mathrm{F}$. taylorii

$F$. whiteleggei $\mathrm{C}$. Muell. ex Rodway $=\mathrm{F}$. pallidus

Funaria calvescens Schwaegr. $=$ F. hygrometrica

F. crispula Hook. f. \& Wils. = F. glabra

F. hygrometrica Hedw. var. sphaerocarpa C. Muell. = F. hygrometrica
F. subnuda Tayl. $=$ F. glabra

F. tasmanica C. Muell. \& Hampe $=$ F. glabra

Glyphomitrium adamsoni Mitt $=$ Ptychomitrium australe

G. commutatum (C. Muell.) Broth. = Ptychomitrium australe

G. howeanum (Hampe) Broth. = Ptychomitrium muelleri

G. muelleri (C. Muell.) Broth. = Ptychomitrium muelleri

Goniobryum pellucidum (Mitt.) Broth. = G. subbasilare

Grimmia alpestris Sommerf. = G. donniana

G. apocarpa Hedw. = Schistidium apocarpum

$G$. campestris Burchell ex Hook $=\mathrm{G}$. laevigata

$G$ cyonicolla Tayl $=\mathrm{G}$ pulvinata var africana

G. flexifolia Hampe $=$ Schistidium rivulare ssp. rivulare

$G$. leiocarpa Tayl. $=\mathrm{G}$. laevigata

G. leucophaea Grev. $=\mathrm{G}$. laevigata

G. mutica Hampe $=$ Schistidium apocarpum

G. pulvinata (Hedw.) Sm. var. obtusa (Brid.) Heub. = G. pulvinata var. africana

Gymnostomum calcareum (Nees) Hornsch. $=\mathrm{G}$. aeruginosum

Haplohymenium brevinerve (Broth.) Broth. $=\mathrm{H}$. pseudotriste

Hedwigia albicans Lindb. $=\mathrm{H}$. ciliata

$H$. juratzkae C. Muell. $=\mathrm{H}$. ciliata

H. microcyathea (C. Muell.) Par. = H. ciliata

Hedwigidium imberbe (Sm.) B.S.G. = Hedwigia integrifolia

H. integrifolium (P. Beauv.) Dix. = Hedwigia integrifolia

Holomitrium dietrichiae $\mathrm{C}$. Muell. $=\mathrm{H}$. perichaetiale

$H$. hodgkinsoniae $\mathrm{C}$. Muell. $=\mathrm{H}$. perichaetiale

H. muelleri $\mathrm{Hampe}=\mathrm{H}$. perichaetiale

$H$. whiteleggei $\mathrm{C}$. Muell. $=\mathrm{H}$. perichaetiale

Homalia acuminata $\mathrm{C}$. Muell. = Thamnobryum pumilum

Hookeria karsteniana Broth. \& Geh. = Cyclodictyon lepidum

Hypnodendron praenitens (Hampe) Mitt. = Pterobryella praenitens

Hypopterygium didictyon $\mathrm{C}$. Muell. $=\mathrm{H}$. rotulatum

$H$. hyalino-limbatum C. Muell. = Lopidium concinnum

$H$. novae-seelandiae $\mathrm{C}$. Muell. $=\mathrm{H}$. rotulatum

H. pallens (Hook. f. \& Wils.) Mitt. = Lopidium concinnum

$H$. scottiae $\mathrm{C}$. Muell. $=\mathrm{H}$. muelleri

Hypnodendron arcuatum non Hedw. $=\mathrm{H}$. vitiense ssp. australe

$H$. arcuatum (Hedw.) Mitt. pro parte $=\mathrm{H}$. spininervium ssp. archeri

$H$. whiteleggei C. Muell. ex Burges $=\mathrm{H}$. vitiense ssp. australe

Hypnum cupressiformis L. var. mossmanii $=$ H.cupressiforme var. mossmanianum

H. mossmanianum $\mathrm{C}$. Muell. $=\mathrm{H}$. cupressiforme var. mossmanianum 
Ischyrodon seriolus (C. Muell.) C. Muell. = I. lepturus Isopterygium austro-pusillum (C. Muell.) Jaeg. = I. minutirameum I. austro-subulatum C. Muell. = I. minutirameum

I. candidum (C. Muell.) Jaeg. $=\mathrm{I}$. albescens

I. novae-valesiae Broth. = Taxithelium novae-valesiae

I. molliculum (Sull) Mitt = I albescens

Juratzkaea leptura (Tayl.) Buck = Ischyrodon lepturus

Lembophyllum brisbanicum C. Muell. = Camptochaete gracilis

L. clandestinum (Hook. f. \& Wils.) Lindb. = L. divulsum

L. vagum (C. Muell.) Lindb. = Camptochaete vaga

Leptodictyum muelleri (Hampe \& C. Muell.) Broth. $=$ L. riparium

Leptodon australis C. Muell. = L. smithii

Leptodontium interruptum (Mitt.) non Broth. $=\mathrm{L}$. paradoxum

Leucobryum brachyphyllum $\mathrm{Hampe}=\mathrm{L}$. candidum

L. speirostichum C. Muell, in Geh. = L. candidum var. pentastichum

L. spirostichum C. Muell. in Mitt. = L. candidum var. pentastichum

L. strictifolium Broth. $=$ L. candidum

L. teysmannianum Dozy \& Molk. = L. candidum

Leucoloma austro-scoparium C. Muell. ex Broth. = Dicranoloma argutum

L. dicarpum (Nees) Broth. = Dicranoloma dicarpum

L. sieberianum (Hornsch.) Jaeg. = Dicnemoloma pallidum

L. serratum Broth. = Dicranoloma serratum

Lopidium plumarium (Mitt.) Hampe = L. concinnum

Macromitrium asperulum Mitt. $=$ M. ligulare

M. brevisetaceum Hampe $=$ M. brachypodium

$M$. carinatum Mitt. = M. involutifolium ssp. ptychomitrioides

$M$. circinocladum $\mathrm{C}$. Muell $=\mathrm{M}$. diaphanum

$M$. daemelii $\mathrm{C}$. Muell, $=\mathrm{M}$. involutifolium

$M$. dimorphum $\mathrm{C}$. Muell. $=\mathrm{M}$. caloblastoides

M. eucalyptorum C. Muell. \& Hampe = Macrocoma tenue ssp. tenue

$M$. geheebii C. Muell. = Macrocoma tenue ssp. tenue

$M$. ligulatulum $\mathrm{C}$. Muell. $=\mathrm{M}$. ligulaefolium

$M$. malacoblastum $\mathrm{C}$. Muell. = M. involutifolium

$M$. microphyllum (Hook. \& Grev.) Brid. = Macrocoma tenue ssp. tenue

$M$. novae-valesiae C. Muell. = Macrocoma tenue ssp. tenue

$M$. prolixum Bosw. = M. microstomum

M. pugionifolium $\mathrm{C}$. Muell. = M. repandum

$M$ pusillum Mitt. $=$ M. archeri

$M$. rodway Dix. $=\mathrm{M}$. longirostre

M. scottiae C. Muell. = M. microstomum

$M$. subbrevicaule Broth. \& Watts = M. brevicaule

$M$. tasmanicum Broth. $=$ M. microstomum
$M$. watsi $i$ Broth. $=$ M. brevicaule

. weissiondes C. Muell. = M. ligulare

M. weymouthii Broth. = M. microstomum

$M$. whiteleggei Broth. \& Geh. = M. repandum

$M$ woollsianum $\mathrm{C}$. Muell $=\mathrm{M}$. ligulaefolium

Meteorium amblyacis (C. Muell.) Mitt. = Papillaria amblyacis

$M$. dimorphum (C. Muell.) Mitt. = Pilotrichella dimorpha

$M$. intricatum Mitt. = Papillaria intricata

M. kermadecensis (C. Muell.) Mitt. = Papillaria crocea

M. trichophoroides (C. Muell.) Mitt. = Barbella enervis

Micromitrium brisbanicum (Broth.) Crosby = Eccremidium brisbanicum

Mielichhoferia ecklonii Hornsch. = M. bryoides

$M$. forsythii Broth. $=\mathrm{M}$. australis

Mittenia rotundifolia (C. Muell.) Par. $=$ M. plumula

Mniadelphis adnatus(Hook f. \& Wils.) Reichdt.= Distichophyllum crispulum

M. amblyophyllus (Hook. f. \& Wils.) Jaeg. = Distichophyllum pulchellum M. crispulus (Hook. f. \& Wils.) C. Muell. = Distichophyllum crispulum $M$. subminutifolius Broth. \& Geh. = Distichophyllum subminutifolium

Mniobryum albicans (Wahlenb.) Limpr. = Pohlia wahlenbergii

M. tasmanicum Broth. = Pohlia wahlenbergii

$M$. wahlenbergii (Web. \& Mohr.) Jenn. = Pohlia wahlenbergii

Mniodendron comosum (Labill.) Lindb. $=$ Hypnodendron comosum

Mnium rostratum auct. non Schrad. = Plagiomnium novae-zealandiae

$M$. longirostrum Brid. = Plagiomnium novae-zealandiae

$M$ novae-valesiae C. Muell in Geh = Plagiomnium novae-zealandiae

Nanomitrium brisbanicum (Broth.) Broth. = Eccremidium brisbanicum Neckera hymenodonta C. Muell. =N. pennata

Orthodontium australe Hook. f. \& Wils. $=0$. lineare ssp. sulcatum

$O$. ovale C. Muell. ex Broth. = O. inflatum

$O$. pallens (Hook. f. \& Wils.) Broth. $=0$. lineare ssp. sulcatum

$O$. sulcatum Hook. f. \& Wils $=0$ lineare ssp. sulcatum

$O$. zetterstedtii C. Muell. = O. lineare ssp. sulcatum

Orthorrhynchium cymbifolioides C. Muell. = O. elegans

$O$. hampeanum $\mathrm{C}$. Muell. $=\mathrm{O}$. elegans

Orthotrichum acroblepharis C. Muell. = O. longithecum

$O$. campbelliae Watts \& Whitel. = O. tasmanicum

O. encalyptaceum C. Muell. $=$ O. tasmanicum

O. laterale Hampe $=\mathrm{O}$. tasmanicum

$O$. sullivanii C. Muell. $=O$. rupestre

Oxyrrhynchium austrinum (Hook. f. \& Wils.) Broth. = Eurhynchium

austrinum

o. speciosum (Brid.) Warnst. = Eurhynchium speciosum 
Papillaria cerina (Hook. f. \& Wils.) Par. = P. flavolimbata $P$ filipendula (Hook. f \& Wils) Jaeg. = P. flexicaulis

$P$. kermadecensis $($ C. Muell. $)$ Jaeg. $=$ P. crocea

$P$. nitidiuscula Broth. $=$ P. nitens

P. scottiae (C. Muell.) Par. = P. flexicaulis

Phascum apiculatum Hook. f. \& Wils. = Acaulon integrifolium

$P$. sullivanii C. Muell. = Tetrapterum sullivani

P. tasmanicum Dix. \& Rodw. = Acaulon crassinervium

Philonotis affinis (Hook.) Jaeg. = Breutelia affinis

P. appressa (Hook. f. \& Wils.) Mitt. = P. scabrifolia

$P$. fertilis (Mitt.) Mitt. = P. tenuis

$P$. pseudomollis (C. Muell.) Jaeg. = P. tenuis

P. remotifolia (Hook. f. \& Wils) Jaeg. $=$ P. scabrifolia

Physcomitrella patens $(\mathrm{Hedw}$.) B.S.G. var. readeri (C. Muell.) Tan $=$

$$
\text { P. readeri }
$$

$P$. subserratum Hampe $=\mathrm{P}$. conicum

Physcomitrium conicum Mitt. = P. pyriforme

Platyhypnidium austrinum (Hook. f. \& Wils.) Fleisch. = Eurhynchium austrinum

Pleuridium austro-subulatum Broth. ex Roth = Eccremidium brisbanicum $P$. gracilentum (Mitt.) Mitt. $=\mathrm{P}$. nervosum

Pogonatum australasicum (C. Muell. \& Hampe) Jaeg. = P. subulatum

Pohlia erythrocaulis Watts \& Whitel. = P. cruda

Polytrichadelphus innovans (C. Muell.) Jaeg. = P. magellanicus

Polytrichum alpinum Hedw. = Polytrichastrum alpinum

$P$. cypellomitrium C. Muell. $=\mathrm{P}$. juniperinum

P. ryparomitrium C. Muell. $=\mathrm{P}$. juniperinum

$P$. sullivanii Hampe $=P$. piliferum

Porotrichum arbusculum $(\mathrm{Sm}$.) Mitt. = Camptochaete arbuscula

P. clandestinum (Hook. f. \& Wils.) Mitt. = Lembophyllum divulsum

P. vagum (C. Muell.) Mitt. = Camptochaete vaga

Pottia brachyodus (Hampe) Jaeg. = Desmatodon convolutus

Pseudoleskea calochlora Broth. \& Watts $=$ P. imbricata

Pseudoleskeopsis imbricata (Hook. f. \& Wils.) Ther. = Pseudoleskea imbricata

Psilopilum pyriforme (Hampe) Jaeg. $=$ P. crispulum

Pterobrvon whiteleggei Broth. = Muellerobryum whiteleggei

Pterogoniella wattsit Broth. $=$ Meiothecium wattsi

Ptervgophyllum dentatum (Hook. f. \& Wils.) Dix. = Achrophyllum dentatum

P. hepaticaefolium (Hampe \& C. Muell.) Jaeg. = Achrophyllum dentatum P. nigellum (Hook. f. \& Wils.) Mitt. = Achrophyllum dentatum $P$. wattsii Broth. = Achrophyllum wattsi

Ptvchomitrium adamsonil (Mitt.) Jaeg. = P. australe

$P$. commutatum (C. Muell.) Par. = P. muelleri
P. serratum (Mitt.) Hook. f. \& Wils. nom. illeg. = P. mittenii

Racomitrium leptostomoides Forster $=$ Racomitrium lanuginosum $R$. sullivanii (C. Muell.) Broth. = Racomitrium crispulum

$R$. symphodon Jaeg. = Racomitrium crispulum

Racopilum convolutaceum (C. Muell.) Reichdt. $=$ R. cuspidigerum var. convolutaceum

$R$. pacificum Besch $=\mathrm{R}$. cuspidigerum var. cuspidigerum

$R$. strumiferum (C. Muell.) Mitt. = R. cuspidigerum var. convolutaceum

Rhacocarpus australis (Hook. f. \& Wils.) Par. $=$ R. purpurascens

R. humboldtii (Hook.) Lindb. $=\mathrm{R}$. purpurascens

Rhaphidorrhynchium amoenum (Hedw.) Fleisch. = Sematophyllum amoenum

$R$. callidioides (C. Muell.) Broth. = Sematophyllum amoenum

R. homomallum (Hampe) Mitt. = Sematophyllum homomallum

$R$. jolliffi $i$ Hook. f. $=$ Sematophyllum jolliffii

R. subfalcatulum (Broth. \& Watts) Mitt. = Pungentella subfalcatulum

$R$. tenuirostre (Hook.) Broth. = Sematophyllum uncinatum

Rhaphidostegium aciculum Broth. ex Dix. = Sematophyllum aciculum

$R$. callidioides (C. Muell.) Jaeg. = Sematophyllum amoenum

$R$. contiguum (Mitt.) Par. = Sematophyllum contiguum

$R$. crassiusculum Brid. = Sematophyllum crassiusculum

$R$. homomallum (Hampe) Broth. = Sematophyllum homomallum

$R$. luciduloides Dix. = Sematophyllum luciduloides

$R$. micropyxis Broth. = Sematophyllum wattsii

R. subfalcatulum (Broth. \& Watts) Broth. = Pungentella subfalcatulum

$R$. tenuirostre (Hook.) Jaeg. = Sematophyllum uncinatum

$R$. wattsii Par. $=$ Sematophyllum wattsii

Rhizogonium geheebii $\mathrm{C}$. Muell. $=\mathrm{R}$. distichum

R. graeffeanum (C. Muell.) Jaeg. $=\mathrm{R}$ distichum

$R$ mnioides (Hook f \& Wils.) Reichdt $=$ Pyrrhobryum mnioides

$R$. parramattense (C. Muell.) Reichdt. = Pyrrhobryum parramattense

$R$. spiniforme Hedw. = Pyrrhobryum spiniforme

Rhodobryum leucocanthum Hampe = Bryum leucocanthum

R. subfasciculatum Hampe = Bryum subfasciculatum

Rhynchostegiella convolutifolia (Hampe) Broth. = Eurhynchium cucullatum

R. cucullata (Mitt.) Dix. = Eurhynchium cucullatum

R. muriculata (Hook. f. \& Wils.) Broth. = Eurhynchium muriculatum

$R$. subconvolutifolia Broth. \& Watts = Eurhynchium cucullatum

Rhynchostegium collatum (Hook. f. \& Wils.) Broth. \& Watts = R tenuifolium

$R$. pseudo-stramineum Mitt. $=\mathbf{R}$. stramineoides

$R$. subclavatum (Hampe) Jaeg $=\mathrm{R}$ tenuifolium

$R$. muriculata (Hook. f. \& Wils.) Broth. = Eurhynchium muriculatum 


\section{R. subconvolutifolia Broth. \& Watts = Eurhynchium cucullatum}

Schlotheimia baileyi Broth. $=$ S. brownii

Sciaromium hispidum (Hook. f. \& Wils.) Par. = Echinodium hispidum Sclerodontium pallidum Hook. $=$ Dicnemoloma pallidum

Sphagnum antarcticum Mitt. $=$ S. australe

S. brotherusii $\mathrm{Warnst} .=\mathrm{S}$. falcatulum

$S$. commutatum Warnst. $=$ S. molliculum

$S$. comosum C. Muell. = S. molliculum

S. cuspidatum Erh. ex Hoffm. = S. falcatulum

$S$. cymbifolioides $C$. Muell. $=$ S. molliculum

S. decipiens Warnst. $=$ S. cristatum

$S$. drepanocladum Warnst. $=$ S. falcatulum

$S$. erythrocalyx auct. non Hampe $=\mathrm{S}$. beccarii

$S$. grandifolium Warnst. $=\mathrm{S}$. cristatum

$S$. magellanicum Brid. $=$ S. cristatum

$S$. novo-zealandicum Mitt. $=$ S. molliculum

$S$. perichaetiale Warnst. $=$ S. australe

$S$. pseudo-rufescens Warnst. $=\mathrm{S}$. molliculm

$S$. serratifolium Warnst. $=$ S. falcatulum

$S$. subbicolor Hampe $=\mathbf{S}$. cristatum

$S$. subcontortum Hampe $=$ S. molliculum

$S$. subsecundum auct. non Nees $=\mathrm{S}$. molliculum

$S$. wardellense Warnst. $=$ S. cristatum

$S$. wattsii Warnst. $=$ S. falcatulum

Spiridens mueller $\mathrm{Hampe}=\mathrm{S}$. vieillardii

Sporledera minutissima (Dix. \& Sainsb.) Sainsb. $=$ Eccremidium whiteleggei

S. whiteleggei (C. Muell.) Broth. $=$ Eccremidium minutum

Stereodon chrysogaster (C. Muell.) Mitt. = Hypnum chrysogaster

S. cupressiformis (Hedw.) Mitt. = Hypnum cupressiforme

S. cupressiformis (Hedw.) Brid. var. mossmanii Mitt. = Hypnum cupressiforme var. mossmanni

S. walterianus (Hampe) Mitt. = Hypnum cupressiforme

Syrrhopodon wattsii Broth. $=$ Calymperopsis wattsii

Tayloria novae-valesiae (C. Muell.) Watts \& Whitel. $=\mathrm{T}$. octoblepharum Thamnium eflagellare Aongstr. = Thamnobryum pandum

T. gracillimum (Hampe) Broth. \& Watts = Thamnobryum pumilum

T. pandum (Hook. f. \& Wils.) Jaeg. = Thamnobryum pandum

T. pumilum (Hook. f. \& Wils.) Lindb. = Thamnobryum pumilum
Thuidiopsis furfurosa (Hook. f. \& Wils.) Fleisch. = Thuidium furfurosum

Thuidium amblystegioides $\mathrm{C}$. Muell. $=\mathrm{T}$. furfurosum

T. hastatum (Mitt.) Reichdt. = T. furfurosum

T. ramentosum (Mitt.) Mitt. = T. cymbifolium

T. suberectum (Hampe) Jaeg. $=\mathrm{T}$. furfurosum

T. unguiculatum (Hook. f. \& Wils.) Fleisch. = T. furfurosum

Tortella calycina (Schwaegr.) Dix. = Barbula calycina

T. nano-tortuosa (C. Muell.) Watts \& Whitel. = T. knightii

T. subflavovirens Broth. \& Watts $=\mathrm{T}$. cirrhata

Tortula acuminata Brid. = Barbula unguiculata

T. asperifolia Broth. $=$ T. crawfordii

T. atro-virens $(\mathrm{Sm}$.$) Lindb. =$ Desmatodon convolutus

T. austro-muralis (C. Muell.) Broth. ex Par. = T. muralis

T. bailevi auct. non Broth. $=$ T. pagorum

T. calvcina (Schwaegr.) Hook. \& Grev. = Tortella calycina

T. chlorotricha Broth. \& Geh. = Barbula chlorotricha

T. flavinervis Dix. = Barbula crinita

T. melbourneana (C. Muell.) Broth. $=$ T. muralis

T. pungens Hook. f. \& Wils. = Barbula crinita

T. subcalycina (C. Muell.) Mitt. = Barbula subcalycina

T. sullivaniana (C. Muell.) Watts \& Whitel. = Aloina sullivaniana

T. torquata (Tayl.) Wils. = Barbula torquata

Trachycarpidium novae-valesiae Broth. = Bryobartramia novae-valesiae

Trachyloma leptopyxis C. Muell. = T. diversinerve

Trachvpus hornschuchii Mitt. = Papillaria crocea

Trematodon abruptus Watts \& Whitel. $=\mathrm{T}$. suberectus

$T$. adaequans Geh. ex Roth $=\mathrm{T}$. suberectus

T. alpinus $\mathrm{J} . \mathrm{H}$. Willis $=\mathrm{T}$. flexipes

Trichostomopsis australasiae (Hook. \& Grev.) Robinson = Barbula australasiae

Trichostomum subdenticulatum C. Muell. = Leptodontium viticulosoides

Webera nutans Hedw = Pohlia nutans

W. tenuifolia Jaeg. $=$ Pohlia tenuifolia

Weissia flavipes Hook. f. \& Wils. = W. controversa

W. truncata $\mathrm{C}$. Muell. $=\mathrm{W}$. controversa

Whiteleggea australis (Broth.) Broth. ex Par. = Hampeella pallens

Wilsoniella compacta Geh. in Watts \& Whitel. = Orthodontium inflatum

Zvgodon brownii Schwaegr. $=Z$. intermedius 


\section{UNSUBSTANTIATED RECORDS OF RECOGNISED SPECIES}

Unsubstantiated records of recognised species amongst Australian collections are included to enable checking to be carried out and possible locations of specimens in overseas Herbaria where early collections e.g. those of C. Müller may be deposited.

\section{Fissidentaceae}

Fissidens ampliretis (C. Muell.) Broth.

F. delicatulus Aongstr. in Watts \& Whitel.

F. perangustatus Broth.

\section{Dicranaceae}

Dicranella stackhousiana (C. Muell.) Broth

[Aongstroemia stackhousiana C. Muell.]

Dicnemoloma fraseri (Mitt.) Ren.

D. imbricatum Broth. \& Geh.

D. strictipilum (C. Muell.) Par.

\section{Encalyptaceae}

Encalypta novae-valesiae Hampe

\section{Pottiaceae}

Tortula crawfordii (Par.) Watts in Watts \& Whitel.

[T. asperifolia Broth.]

T. parramattana Mitt.

[Barbula parramattana (Mitt.) Kind.]

\section{Funariaceae}

Physcomitrium flaccidum Mitt.

\section{Bryaceae}

Bryum baeuerlenii $C$. Muell.

B. brachycladulum $C$. Muell.

B. ischyrrhodon C. Muell.

\section{Bartramiaceae}

Breutelia baeuerlenii (C. Muell.) Watts \& Whitel.

B. leptodontoides (C. Muell.) Watts \& Whitel.

B. Ionchopelma (C. Muell.) Watts \& Whitel.

\section{Ptychomniaceae}

Glyphothecium perrotii C. Muell.

Neckeraceae

Thamnobryum (Thamnium) novae-valesiae Kindb.

Amblystegiaceae

Amblystegium novae-valesiae Broth. \& Watts

\section{Brachytheciaceae}

Eurhynchium asperipes (Mitt.) Dix.

\section{Polytrichaceae}

Polytrichum recurvipilum $C$. Muell.

\section{NOMINA NUDA}

The purpose of listing nomina nuda is to encourage examination of the material to determine which names should be absorbed into the synonymy and which require validation as new species. The list includes only those names in literature (not names in scheda) which appear in Index Muscorum.

The number of collections $(>3$ or $\leq 3)$ to which the name has been applied and the localities represented by these specimens are included to indicate whether the name has been widely used or not.

O absent $\bullet 3$ collections $\star \leqslant 3$ collections 
Tortella angusticaulis Watts $\&$ Whitel.

T. aristatula Broth. in Watts \& Whitel.

Tortula brunnea Broth. \& Watts

T. mollissima Broth. in Watts \& Whitel.

T. rotundata Geh. in Watts \& Whitel.

Triquetrella albicuspis Broth. in Broth. \&

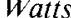

Weissia tortelloides Broth. in Watts \& Whitel

W. whiteleggeana C. Muell. in Watts \&

$$
\text { Whitel. }
$$

\section{FUNARIACEAE}

Funaria pilifera (Mitt.) Broth.

F. woollsianus C. Muell. in Watts \& Whitel.

Physcomitrium novae-valesiae Broth. in

Watts \& Whitel.

\section{BRYACEAE}

Bryum leucoloma Broth. in Burg. . . . . . .

B. pseudotriquetrum Broth in Burg. .. .

B subinclinatum Watts \& White

B. subleptothecium C. Muell.

\section{BARTRAMIACEAE}

Philonotis harrisii Geh. in Watts \& Whitel

P. fontanoides Broth. \& Watts

P. micropteris Broth. in Wats \& Whitel.

P. percapillaris C. Muell. in Watts \& Whitel

P. subsimplex Watts \& Whitel.

P. uncinatula $C$. Muell.
P. whiteleggei Watts \& Whitel.

. $\quad \ldots \quad \ldots$

\section{ORTHOTRICHACEAE}

Macromitrium hartmanii C. Muell. ex Kindb.

M. richmondiae Broth. in Watts \& Whitel

(M. subhemitrichoides Broth.)

M. sheareri Broth

(M. microblastum Broth.)

M. rupicola C. Muell. in Watts \& Whitel

$\begin{array}{llllllllllllll}0 & 0 & \star & 0 & 0 & 0 & 0 & 0 & 0 & 0 & 0 & 0 & 0 & 0 \\ 0 & 0 & 0 & 0 & 0 & 0 & 0 & 0 & 0 & 0 & 0 & 0 & 0 & 0 \\ 0 & 0 & 0 & 0 & 0 & 0 & 0 & 0 & 0 & 0 & 0 & 0 & 0 & 0 \\ 0 & 0 & \star & 0 & 0 & 0 & 0 & 0 & 0 & 0 & 0 & 0 & 0 & 0 \\ 0 & 0 & \star & 0 & 0 & 0 & 0 & 0 & 0 & 0 & 0 & 0 & 0 & 0 \\ 0 & 0 & 0 & 0 & 0 & 0 & 0 & 0 & 0 & 0 & 0 & 0 & 0 & 0 \\ 0 & 0 & 0 & 0 & 0 & 0 & 0 & 0 & 0 & 0 & 0 & 0 & 0 & 0 \\ 0 & 0 & 0 & 0 & 0 & 0 & 0 & 0 & 0 & 0 & 0 & 0 & 0 & 0\end{array}$

48. CRYPHAEACEAE

Cryphaea viridissima $C$. Muell. .......

O

$\star \quad 000000$

\section{LEMBOPHYLLACAE}

Camptochaete deflexula $C$. Muell. in Burg.

All specimens with these names have been examined and correctly identified

$$
\text { by Vitt \& Ramsay. }
$$

These names remain as nomina nuda. 


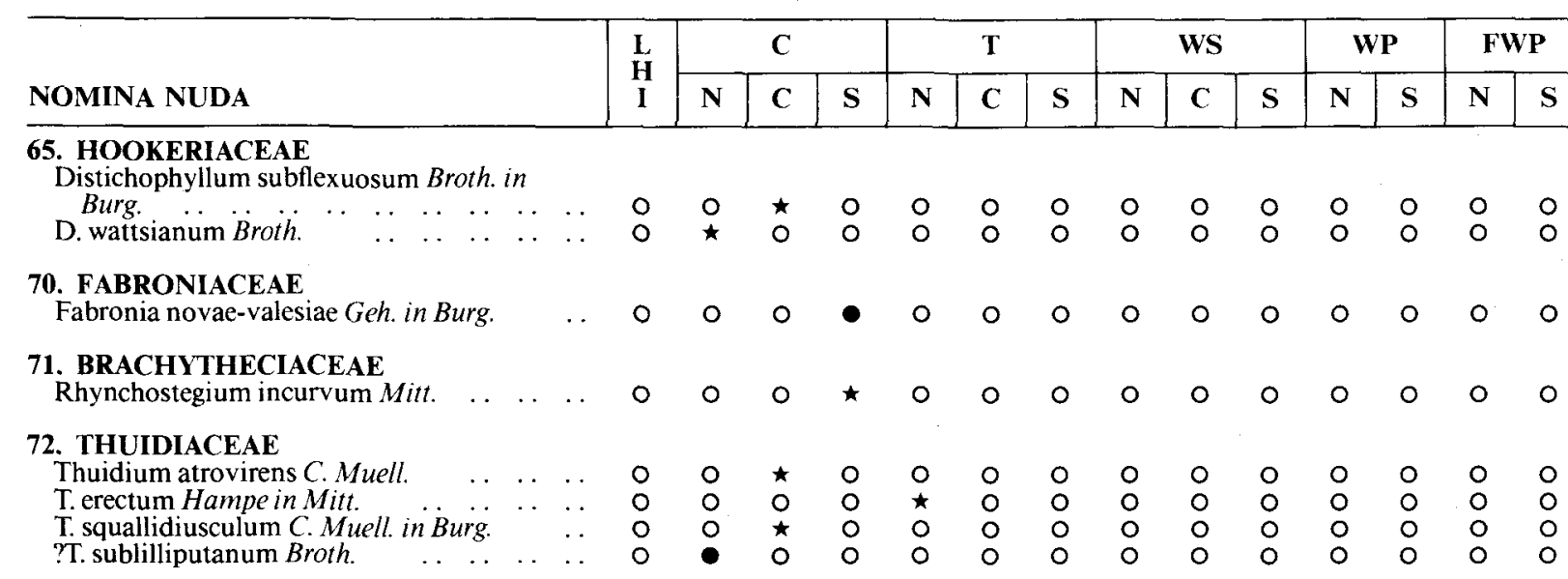

\section{AMBLYSTEGIACEAF}

Amblystegium campylopodioides Broth. in Burg.

A. orbiculare Jaeg.

(Porotrichum orbiculare Miti.)

76. PlagiotheCiaCEAE

Plagiothecium amblyostomum C. Muell. in

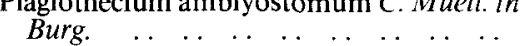

P. howeanum Jaeg.

\section{SEMATOPHYLLACEAE}

Acanthocladium sericeum Broth. in Burg. Rhaphidostegium brachytheciella C. Muell.

$$
\text { in Burg. }
$$

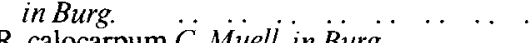

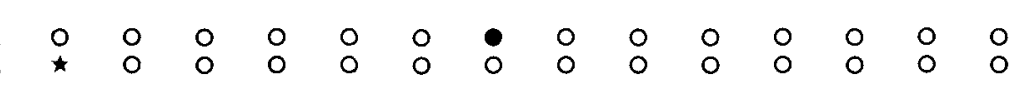

78. HYPNACEAE

Hyocomium brachythecioides Broth. ... .

Hypnum pseudo-demissum $C$. Muell

Isopterygium umbillicatum Mitt.

I. vinde-patens $C$. Muell. in Burg.

$\begin{array}{llllllllllllll}\circ & \circ & \star & \circ & 0 & 0 & \circ & 0 & 0 & 0 & 0 & 0 & 0 & 0 \\ \star & 0 & 0 & 0 & 0 & 0 & 0 & 0 & 0 & 0 & 0 & 0 & 0 & 0\end{array}$

Pylaisia pseudo-homomalla Jaeg. $\ldots \quad \ldots \quad$

$\begin{array}{llllllll}0 & 0 & 0 & 0 & 0 & 0 & 0 & 0 \\ 0 & 0 & 0 & 0 & 0 & 0 & 0 & 0 \\ 0 & 0 & 0 & 0 & 0 & 0 & 0 & 0\end{array}$

$\begin{array}{llllllllllllll}0 & \star & 0 & 0 & 0 & 0 & 0 & 0 & 0 & 0 & 0 & 0 & 0 & 0 \\ 0 & 0 & 1 & 0 & 0 & 0 & 0 & 0 & 0 & 0 & 0 & 0 & 0 & 0 \\ 0 & 0 & \star & 0 & 0 & 0 & 0 & 0 & 0 & 0 & 0 & 0 & 0 & 0 \\ 0 & 0 & 0 & 0 & 0 & 0 & 0 & 0 & 0 & 0 & 0 & 0 & 0 & 0 \\ 0 & 0 & 0 & \star & \star & 0 & 0 & 0 & 0 & 0 & 0 & 0 & 0 & 0\end{array}$




\section{UNSUBSTANTIATED NOMINA NUDA}

The following names have appeared in literature but no specimens have yet been located with these names.

\section{Fissidentaceae}

Fissidens applanatus $C$. Muel

F. perangustus $C$. Muell.

F. porosus Hampe

F. whiteleggeanus $C$. Muell.

F. woollahrae Broth

\section{Dicranaceae}

Holomitrium novae-valesiae C. Muell. in Geh.

\section{Leucobryaceae}

Leucobryum australe Watts \& Whitel.

\section{Funariaceae}

Entosthodon cuspidatus Kiaer in C. Muell.

Physcomitrium torquescens $C$. Muell.

\section{Bryaceae}

Pohlia pseudopallescens Hampe in C. Muell.

\section{Bartramiaceae}

Bartramia austro-alpina C. Muell.

Breutelia crawfordii Watts \& Whitel.

B. luteola C. Muell. ex Broth. in Geh.

Philonotis austrolutea C. Muell. in Jaeg.

P. fontanoides Broth. \& Watts

\section{Lembophyllacea}

Porotrichum decurvatum Mitt.

Brachytheciaceae

Rhynchostegium latifolium Geh.

R. obtusissimum Geh.

R. parramattense Mitt

\section{Sematophyllaceae}

Acanthocladium macro-extenuatum C. Muell. in Burg.

A. pseudo-extenuatum C. Muell. in Burg.

A. sub-extenuatum C. Muell. in Burg.

Rhaphidostegium tingiringense Geh. in Burg.

\section{Hypnaceae}

Hypnum subextenuatum $C$. Muell. 


\section{BIBLIOGRAPHY}

The following articles have been used in preparing the Census list and related papers. References specific to only one of the papers are given separately with the paper if not included here.

Allison, K.W. (1971). Mosses discovered in New Zealand since the publication of Sainsbury's 'A Manual of the New Zealand Mosses' in 1955. New Zealand J.Bot. 9: 672-673.

Allison, K.W. \& Child, J. (1971) 'The Mosses of New Zealand.' (Univ. of Otago Press: Dunedin.)

Anderson, R.H. (1961). Introduction. Contr. New South Wales Natl. Herb., Fl. Ser. Nos 1-18: 1-15.

Anderson, R.H. (1968). 'The Trees of New South Wales.' (Govt Printer: Sydney.) Edn 4.

Ando, H. (1972a). Studies on the genus Hypnum Hedw. I. J. Sci. Hiroshima Univ., Ser. B, Div. 2. Bot. 14: 53-73.

Ando, H. (1972b). Distribution and speciation in the genus Hypnum in the circumPacific region. J. Hattori Bot. Lab. 35: 68-98.

Ando, H. (1973). Studies on the genus Hypnum Hedw. II. J. Sci. Hiroshima Univ., Ser. B, Div. 2. Bot. 14: 165-207.

Ando, H. (1977). Studies on the genus Hypnum Hedw. III. J. Sci. Hiroshima Univ., Ser. B, Div. 2. Bot. 16(1): 1-46.

Ando, H. (1979). A phytogeographical account of Hypnum cupressiforme in Japan. Bull. Phytosoc. Soc. Japan 16: 339-348.

Ando, H. (1980). Speciation and distribution of the Hypnum cupressiforme complex (Musci). Paper delivered at ICSEB - II Vancouver 1980.

Ando, H. (1982). Hypnum in Australasia and the Pacific. J. Hattori Bot. Lab. 52: 93-106.

Ando, H. \& Matteri, C.M. (1981). Patagonian Bryophytes VII. Hypnum revolutum (Mitt.) Lindb. new to South America, with notes on Hypnum of the Transecta zone. Lindbergia 8(1): 60-64.

Andrews, A.L. (1945). Taxonomic notes V. The genus Tetrapterum. Bryologist 48: 190-193.

Andrews, A.L. (1947). Taxonomic notes VI. The Leucobryaceae. Bryologist 50: 319-326.

Andrews, A.L. (1949). Taxonomic notes VIII. The genus Acrocladium. Bryologist 52: $72-77$.

Andrews, A.L. (1951). Taxonomic notes X. The family Leptostomaceae. Bryologist 54(4): 217-223.

Ashton, D.H. \& McRae, R.F. (1970). Distribution of epiphytes on beech trees at Mt. Donna Buang, Victoria. Victorian Naturalist 87: 253-261.

Bailey, F.M. (1883). 'A Synopsis of the Queensland Flora; containing both the Phaenogamous and Cryptogamous Plants'. (Govt Printer: Brisbane.) pp.724-736.

Bailey, F.M. (1886). 'A Synopsis of the Queensland Flora. First Supplement.' (Govt Printer: Brisbane.) pp.65-69.

Bailey, F.M. (1888). 'A Synopsis of the Queensland Flora. Second Supplement.' (Govt Printer: Brisbane.) pp.67-73.

Bailey, F.M. (1890a). 'A Synopsis of the Queensland Flora. Third Supplement.' (Govt Printer: Brisbane.) pp.95-100.

Bailey, F.M. (1890b). 'Catalogue of the Indigenous and Naturalised Plants of Queensland.' (Govt Printer: Brisbane.) pp.60-63, 114-115.

Bailey, F.M. (1891-6). Contributions to the Queensland flora. Botany Bull. Dep. Agric. Queensland (1891); 2: 23-28 (1891); 4: 21 (1891), 5: 29 (1892), 8: 87-90 (1893); 13: 17-19 (1896).

Bailey, F.M. (1898). Contributions to the Flora of Queensland. Queensland Agric. J. 3(3): 203. 
Bailey, F.M. (1913). 'Comprehensive Catalogue of Queensland Plants.' (Govt Printer: Brisbane.) pp.656-672.

Bartram, E.B. (1933). Manual of Hawaiian mosses. Bull. Bernice P. Bishop Mus. 101: 1-275.

Bartram, E.B. (1944). Henry Neville Dixon. Bryologist 47(4): 137-144.

Bartram, E.B. (1951). West Australian mosses. Trans. Brit. Bryol. Soc. 1(5): $465-470$.

Bartram, E.B. (1952). North Queensland mosses collected by L.J. Brass. Farlowia 4(1): 235-247.

Bastow, R.A. (1886a). Tasmanian mosses, their identification, etc. Pap. \& Proc. Roy. Soc. Tasmania 1885: 318-320.

Bastow, R.A. (1886b). Moss flowers, split-moss, bog-moss, and earth-moss. Pap. \& Proc. Roy. Soc. Tasmania 1885: 337-341.

Bastow, R.A. (1886c). Mosses of Tasmania (continued), Tribe 2, Weissiae. Pap. \& Proc. Roy. Soc. Tasmania 1885: 395-399.

Bastow, R.A. (1887). Tasmanian mosses. Pap. \& Proc. Roy. Soc. Tasmania 1886: 38-102.

Boswell, H. (1892). New exotic mosses. J. Bot. 30: 97-99.

Bremer, B. (1980a). A taxonomic revision of Schistidium (Grimmiaceae, Bryophyta) 1. Lindbergia 6(1): 1-16.

Bremer, B. (1980b). A taxonomic revision of Schistidium (Grimmiaceae, Bryophyta) 2. Lindbergia 6(2): 89-117.

Bremer, B. (1981). A taxonomic revision of Schistidium (Grimmiaceae, Bryophyta) 3. Lindbergia 7(2): 73-90.

Bridel-Brideri, S.E. von (1826-7). 'Bryologia Universa seu Systematica ad Novam Methodum Dispositio, Historia et Descriptio Omnium Muscorum Frondosorum Hucusque Cognitorum cum Synonymia ex Auctoribus Probatissimis.' (Barth: Leipzig.) 2 vols.

Brotherus, V.F. (1890). Some new species of Australian mosses described. I. Oefvers. Forh. Finska Vetensk.-Soc. 33: 1-22.

Brotherus, V.F. (1893). Some new species of Australian mosses described. II. Oefvers. Forrh. Finska Vetensk.-Soc. 35: 34-56.

Brotherus, V.F. (1895). Some new species of Australian mosses described. III. Oefvers. Förh. Finska Vetensk.-Soc. 37: 149-172.

Brotherus, V.F. (1898). Some new species of Australian mosses described. IV. Oefvers. Forrh. Finska Vetensk.-Soc. 40: 159-193.

Brotherus, V.F. (1900). Some new species of Australian mosses described. V. Oefvers. Forh. Finska Vetensk.-Soc. 42: 91-129.

Brotherus, V.F. (1916). Descriptions of some new species of Australian, Tasmanian, and New Zealand mosses. VI. Proc. Linn. Soc. New South Wales 41(3): $575-596$.

Brotherus, V.F. (1924-5). Unterklasse Bryales II. Spezieller Teil. In Engler, A. and K. Prantl. 'Die naturlichen Pflanzenfamilien.' Vol. 10: 143-478. Vol. 11. 1-542. (Engelmann: Leipzig.) Edn 2.

Brotherus, V.F. \& Watts, W.W. (1912). The mosses of the Yarrangobilly Caves District, N.S.W. Proc. Linn. Soc. New South Wales 37(2): 363-382.

Brotherus, V.F. \& Watts, W.W. (1915). The mosses of Lord Howe Island. Proc. Linn. Soc. New South Wales 40(2): 363-385.

Brotherus, V.F. \& Watts, W.W. (1918). The mosses of North Queensland. Proc. Linn. Soc. New South Wales 43: 544-567.

Brough, P., McLuckie, J. \& Petrie, H.K. (1924). An ecological study of the flora of Mount Wilson. Proc. Linn. Soc. New South Wales 49: 475-479.

Brown, R. (1811). Some observations on the parts of fructifications in mosses, with characters and descriptions of two new genera of that order. Trans. Linn. Soc. London 10: 312-324.

Bruch, P., Schimper, W.P. \& Guembel, T. (1836-55). 'Bryologia Europea seu Genera Muscorum European Monographice Illustrata.' (Schweizerbarth: Stuttgart.) 6 vols. 
Bruggeman-Nannenga, M.A. (1978). Notes on Fissidens I and II. Remarks on some species of Fissidens bryoides complex. Proc. K. Ned. Akad. Wet. Ser. C. 81: $387-402$.

Bruggeman-Nannenga, M.A. (1979). The section Pachylomidium (genus Fissidens) II - The species of Central America, temperate South America, including the Andes, Australia, New Zealand and New Guinea. Proc. $K$. Ned. Akad. Wet. Ser. C. 82: 11-27.

Buck, W.R. (1977). A taxonomic revision of Juratzkaea Lor. and Juratzkaea gen. nov. Rev. Bryol. Lichénol. 43(3): 309-325.

Buck, W.R. (1979a). A re-interpretation of the Fabroniaceae: additions and corrections. J. Hattori Bot. Lab. 47: 45-56.

Buck, W.R. (1979b). A re-evaluation of the Bruchiaceae with the description of a new genus. Britonnia 31(4): 469-473.

Buck, W.R. (1980a). A generic revision of the Entodonaceae. J. Hattori Bot. Lab. 48: 71-160.

Buck, W.R. (1980b). A re-interpretation of the Fabroniaceae: additions and corrections. J. Hattori Bot. Lab. 48:71-160.

Buck, W.R. (1980c). Aminadversions on Ptergynandrium and special commentary on Forrstroemia and Leptogynandrium. Bryologist 83(4): 451-465.

Buck, W.R. (1981a). A review of Cheilothela (Ditrichaeceae). Brittonia 33(3): 453-456.

Buck, W.R. (1981b). A re-interpretation of the Fabroniaceae III - Anacomptodon \& Fabronidium revisited, Mamillariella, Helicodontiadelphus \& Bryobartlettia gen. nov. Brittonia 33(3): 473-481.

Buck, W.R. \& Crum, H. (1978). A re-interpretation of the Fabroniaceae with notes on selected genera. J. Hattori Bot. Lab. 44: 347-369.

Burbidge, N.T. \& Gray, M. (1965). The plants of the Australian Capital Territory. Tech. Pap. Div. Pl. Ind. CSIRO, Aust. 21: 16-30.

Burbidge, N.T. \& Gray, M. (1970). 'Flora of the Australian Capital Territory.' (A.N.U. Press: Canberra.)

Burges, A. (1932). Notes on the mosses of New South Wales. I. Additional records and description of a new species of Buxbaumia. Proc. Linn. Soc. New South Wales 57: 239-244.

Burges, A. (1935). Notes on the mosses of New South Wales. II. Additional records. Proc. Linn. Soc. New South Wales 60: 83-93.

Burges, A. (1949). The genus Dawsonia. Proc. Linn. Soc. New South Wales 74: 83-96.

Burges, A. (1952). Census of the N.S.W. Mosses. Botany School, University of Sydney. MS. 84pp.

Burges, A.B. \& Johnston, R.D. (1953). The structure of a New South Wales subtropical rainforest. J. Ecol. 41: 72-83.

Cardot, J. (1908). Notes bryologiques IV. Le Dicranum Novae-Hollandiae Hsch. Bull. Herb. Boissier. Sér. 2, 8: 173-174.

Catcheside, D.G. (1958). Some bryophytes collected in Arnhem Land. In Specht, R.L. and C.P. Mountford (eds), 'Records of the American-Australian Expedition to Arnhem Land.' (Melbourne Univ. Press: Melbourne.) Vol. 3, p.169.

Catcheside, D.G. (1967). Tortula pagorum in Australia. Muelleria 1(3): 227-230.

Catcheside, D.G. (1980). 'Mosses of South Australia.' (Govt Printer: South Australia.)

Catcheside, D.G. \& Stone, I.G. (1980). The peristome of Ischyrodon lepturus (Tayl.) Schelpe. J. Bryol. 11: 99-103.

Catcheside, D.G. (1982). The geographical affinities of the mosses of South Australia. J. Hattori Bot. Lab. 52: 57-64.

Chuang, C.C. (1973). A moss flora of Taiwan exclusive of essentially pleurocarpous families. J. Hattori Bot. Lab. 37: 419-509. 
Churchill, S.P. (1982). A phylogenetic analysis, classification and synopsis of the genera of the Grimmiaceae (Musci). In Funk, V.A. and D.R. Brooks (eds), 'Advances in Cladistics.' (New York Botanical Garden: New York.) pp.127-144.

Churchill, S.P. \& Buck, W.R. (1982). A taxonomic investigation of Leptotheca (Rhizogoniaecae). Brittonia 34(1): 1-11.

Clarke, G.C.S. (1973). Type specimens in Manchester Museum Herbarium. Musci. Mus. Publ. Manchester Mus. New Series, 2(73): 1-20.

Clifford, H.T. (1952). Victorian Musci Part I: Introduction and Andreaeceae. Proc. Roy. Soc. Victoria 64(1): 4-9.

Clifford, H.T. (1955). On the distribution of Rhacomitrium crispulum (H.f. \& W.) H.f. \& W. Bryologist 58: 330-334.

Clifford, H.T. \& Willis, J.H. (1951-2). The genera of Victorian mosses, and new records of species for the State. Victorian Naturalist 68(8-9): 135-138, $151-158$.

Corley, M.F.V. (1980). The Fissidens viridulus complex in the British Isles and Europe. J. Bryol. 11(2): 191-208.

Costin, A.B. (1954). 'A Study of the Ecosystems of the Monaro Region of New South Wales with Special Reference to Soil Erosion,' (Grovt Printer: Sydney.)

Crosby, M.R. (1968). Micromitrium Aust., an earlier name for Nanomitrium Lindb. Bryologist 71: 114-117.

Crosby, M.R. (1972). Pterygophyllum Brid. - nomen rejiciendum et illegitimum. Taxon 21: 205-209.

Crosby, M.R. (1974). Toward a revised classification of the Hookeriaceae (Musci). J. Hattori Bot. Lab. 38: 129-141.

Crosby, M.R. (1977). Index Muscorum Supplementum - 1974-1975. Taxon 26(2/3): 285-307.

Crosby, M.R. (1979). Index Muscorum Supplementum - 1976-1977. Taxon 28(1,2/3): 237-264.

Crosby, M.R. \& Bauer, C.R. (1981). Index Muscorum Supplementum - 1978-1979. Taxon 30: 667-693.

Crosby, M.R., Herrnstadt, I. \& Heyn, C.C. (1980). New data on the moss genus Gigaspermum. Bryologist 83 (4): 536-541.

Crosby, M.R. \& Magill, R.E. (1981). 'A Dictionary of Mosses.' (Missouri Botanical Garden: St. Louis.)

Crum, H. (1971). Nomenclatural changes in the Musci. Bryologist 74: 165-174.

Crum, H. (1973). A taxonomic account of the Erpodiaceae. Nova Hedwigia 23: 201-224.

Crum, H. (1976). 'Mosses of the Great Lakes Forest.' (University Herbarium, Univ. Michigan: Ann Arbor.) Revised edn.

Crundwell, A. C. (1970). Notes on the nomenclature of British Mosses. I. Trans. Brit. Bryol. Soc. 6(1): 133-138.

Crundwell, A. C. (1971). Notes on the nomenclature of British Mosses. II. Trans. Brit. Bryol. Soc. 6(2): 323-326.

Crundwell, A.C. \& Nyholm, E. (1964). The European species of the Bryum erythrocarpum complex. Trans. Brit. Bryol. Soc. 4(4): 597-637.

Delgadillo, M.C. (1973). A new species, nomenclatural changes, and generic limits in Aloina, Aloinella, and Crossidium (Musci). Bryologist 76: 271-277.

Delgadillo, M.C. (1975). Taxonomic revision of Aloina, Aloinella and Crossidium (Musci). Bryologist 78: 245-303.

Dixon, H.N. (1912). On some mosses of New Zealand. J. Linn. Soc., Bot. 40: 433-459.

Dixon, H.N. (1913-29). 'Studies in the Bryology of New Zealand, with Special Reference to the Herbarium of Robert Brown, of Christchurch, New Zealand.' (Govt Printer: Wellington.) 6 parts. 
Dixon, H.N. (1915). New and rare Australasian mosses, mostly from Mitten's herbarium. Bull. Torrey Bot. Club 42: 93-110.

Dixon, H.N. (1916). Miscellanea bryologica.V. J. Bot. 54: 352-359.

Dixon, H.N. (1924a). 'The Student's Handbook of British Mosses.' (Sumfield and Day: Eastbourne.) Edn 3.

Dixon, H.N. (1924b). Miscellanea bryologica. IX. J. Bot. 62: 228-236.

Dixon, H.N. (1929). Critical mosses. Rev. Bryol. n.s. 2: 21-29.

Dixon, H.N. (1932). Classification of mosses. In Verdoorn, F. (ed.), 'Manual of Bryology.' (Martinus Mijhoff: The Hague.) pp. 397-412.

Dixon, H.N. (1936). Decas generum novorum muscorum. J. Bot. 74: 1-10.

Dixon, H.N. (1937). Notulae bryologicae. I. J. Bot. 75: 121-129.

Dixon, H.N. (1938). Mosses in North Queensland. N. Queensland Naturalist 6: 2 , 4.

Dixon, H. N. (1942). Additions to the mosses of North Queensland. Proc. Roy. Soc. Queensland 53(2): 23-40.

Dixon, H.N. (1950). Notes on the moss collections of the Royal Botanic Garden Edinburgh. Part II. Notes Roy. Bot. Gard. Edinburgh 20: 93-102.

Doignon, P. (1953). Les Stereodon exotiques. Rev. Bryol. Lichénol. 22: 34-51.

Dozy, F. \& Molkenboer, J.H. (1844). 'Bryologica Javanica.' (Sythoff: Leiden.) Vol. 2, pp. 1-238.

Duby, J.E. (1870). Choix de Cryptogames exotiques nouvelles ou mal connues. Mém. Soc. Phys. Geneve 20: 351-364.

During, H.J. (1977). A taxonomical revision of the Garvaglioideae (Pterobryaceae, Musci.) Bryophytum Bibliotheca 12: 1-244.

Fife, A.J. (1979). Taxonomic observations on three species of North American Funaria. Bryologist 82(2): 204-214.

Fife, A.J. (1980). The affinities of Costesia and Neosharpiella and notes on the Gigaspermaceae (Musci). Bryologist 83 (4): 466-476.

Fleischer, M. (1902-8). 'Die Musci der Flora von Buitenzorg.' (E.J. Brill: Leiden.) 4 vols.

Fleischer, M. (1906). Neue Familien, Gattungen und Arten der Laubmoose. I. Hedwigia 45: 53-87.

Fleischer, M. (1913). Seltene sowie einige neue indische Archipelmoose nebst Calymperopsis gen. nov. Biblioth. Bot. 80: 1-11.

Fleischer, M. (1914). Kritische Revision von Carl Müllerschen Laubmoosgattungen I. Hedwigia 55: 280-285.

Fleischer, M. (1918). Kritische Revision von Carl Müllerschen Laubmoosgattungen II. Hedwigia 59: 212-219.

Fleischer, M. (1920a). Kritische Revision von Carl Müllerschen Laubmoosgattungen III. Hedwigia 61: 402-408.

Fleischer, M. (1920b). Natürliches System der Laubmoose. Hedwigia 61: 390-400. Fleischer, M. (1922). Kritische Revision der Carl Müllerschen Laubmoosgattungen IV. Hedwigia 63: 209-216.

Forsyth, W. (1899). Contribution to a knowledge of the mosses of New South Wales. Proc. Linn. Soc. New South Wales 24: 674-686.

Frahm, J-P. (1978). Taxonomische notizen zur Gattung Campylopus. Nova Hedwigia 30: 835-849.

Frahm, J-P. (1981). Taxonomische notizen zur Gattung XI. Nova Hedwigia 34: 391-395.

Frahm, J-P. (1982). Taxonomische notizen zur Gattung Campylopus XII. Cryptogamie Bryologie Lichenologie 3(1): 59-65.

Fraser, L. \& Vickery, J.W. (1938). The ecology of the upper Williams River and Barrington Tops districts. II. The rain-forest formations. Proc. Linn. Soc. New South Wales 63: 139-184.

Gangulee, H.C. (1969-79) 'Mosses of Eastern India and Adjacent Regions.' 5 vols. (Privately published: Calcutta.)

Geheeb, A. (1876). Sur une petite collection de mousses d'Australie recoltées par un amateur. Rev. Bryol. 3:2-4. 
Geheeb, A. (1877). Sur quelques nouvelles espèces de mousses d'Australie et d'Afrique. Rev. Bryol. 4: 43.

Geheeb, A. (1896). Essai d'une monographie du genre Dawsonia, par le Dr. C. Schliephacke et A. Geheeb. Rev. Bryol. 23(4): 73-79.

Geheeb, A. (1897). Nouvelles additions aux flores bryologiques de l'Australie et de la Tasmanie. Rev. Bryol. 24(5): 65-79.

Geheeb, A. \& Hampe, E. (1881). See Hampe and Geheeb (1881).

Goebel, K. (1906). Archegoniatenstudien 10. Beiträge zur Kenntnis australischer und neuseelandischer Bryophyten. Flora, Jena 96: 1-202.

Gradstein, S.R. \& Sipman, H.J.M. (1978). Taxonomy and world distribution of Campylopus introflexus and $C$. pilifer $(=\mathrm{C}$. polytrichoides): a new synthesis. Bryologist 81: 114-121.

Greene, S.W. (1981). Retrieval works useful to the bryological taxonomist. Occas. Pap. Farlow Herb. 16: 83-99.

Grout, A.J. (1928-40). 'Moss Flora of North America North of Mexico.' (Privately published: Newfane; repro. 1972, Hafner: New York.) 3 vols.

Hampe, E. (1844). 'Icones Muscorum Novorum vel Minus Cognitorum.' (Henry and Cohen: Bonn.)

Hampe, E. (1846-7). Musci Dill. In Lehmann, J.G.C.(ed.), 'Plantae Pressianae sive Enumeratio Plantarum Quas in Australasia Occidentali et Meridionalioccidentali Annis 1838-41 Collegit Ludovicus Preiss.' (Meissner: Hamburg.) Vol. 2. pp. 113-120.

Hampe, E. (1856). Plantae Muellerianae. Musci frondosi in Australasia felici lecti (continuatio). Linnaea 28: 203-215.

Hampe, E. (1860). Muscorum frondosorum Florae Australasiae auctore Dr. F. Muller mox edendae species novas. Linnaea 30: 623-646.

Hampe, E. (1870). Species muscorum novas ex Herbario Melbourneano Australiae. Linnaea 36(5): 513-526.

Hampe, E. (1871). Appendix: Hypna duo Australiae adnumerat. Linnaea 37: 143-162.

Hampe, E. (1872). Musci novi Australiae ex Herbario Melbournio, a doctore F. von Müller missi. Linnaea 37: 513-519.

Hampe, E. (1874). Species muscorum novas ex Herbario Melbourneo (sic) Australiae. Linnaea 38: 661-672.

Hampe, E. (1876). Musci novi Musei Melbournei. Continuatio. Linnaea 40: 301-326.

Hampe, E. (1880). Musci Frondosi Australiae continentalis, praesertim e Baronis de Mueller collectionibus. In Mueller, F. 'Fragmenta Phytographiae Australiae.' (Govt Printer: Melbourne.) Vol. 11, Suppl., pp. 45-52.

Hampe, E. \& Geheeb, A. (1881). Musci frondosi in Tasmania et Nova-Seelandia a Dr. O. Beccari, anno 1878, lecti. Rev. Bryol. 8: 25-28.

Hedenas, L. (1981). Orthodontium lineare. Svensk Bot. Tidskr. 75: 157-161.

Hedwig, J. (1801). 'Species Muscorum Frondosorum Descriptae et Tabulis Aenis LXXVII Coloratis Illustratae.' (Barth: Leipzig.)

Hirohama, T. (1977a). Spore morphology of bryophytes observed by scanning electron microscope. II.Bartramiaceae. Bull. Nat. Sci. Mus. Tokyo 3(1): $37-44$.

Hirohama, T. (1977b). Spore morphology of bryophytes observed by scanning electron microscope. III. Orthotrichaceae. Bull. Nat. Sci. Mus. Tokyo 3(3): 113-122.

Hirohama, T. (1978). Spore morphology of bryophytes by scanning electron microscope. IV. Grimmiaceae. Bull. Nat. Sci. Mus. Tokyo 4(1): 33-42.

Hirohama, T. \& Iwatsuki, Z. (1980). Surface ornamentation of rhizoids of species of Bartramiaceae (Musci). J. Hattori Bot. Lab. 48: 259-275.

Holmgren, P.K. \& Keuken, W. (1964). 'Index Herbariorum.' (Int. Bur. Pl. Taxon.: Utrecht.) Edn 5.

Hooker, J.D. (1855). 'The Botany of the Antarctic Voyage. . ' (Reeve: London.) Vol. II [Flora Novae-Zelandiae], Part 2. 
Hooker, J. D. \& Wilson, W. (1844). Musci Antarctici; being characters with brief descriptions of the new species of mosses discovered during the voyage of H.M. Discovery ships "Erebus" and "Terror" in the southern circumpolar regions, together with those of Tasmania and New Zealand. London J. Bot. 3: 533-556.

Hooker, W.J. (1818-20). 'Musci Exotici; Containing Figures and Descriptions of New or Little Known Foreign Mosses and Other Cryptogamic Subjects.' (Longmans: London.) 2 vols.

Hooker, W.J. \& Greville, R.K. (1824a). Sketch of the characters of the species of mosses, belonging to the genera Orthotrichum. Edinburgh J. Sci. 1: 110-133.

Hooker, W.J. \& Greville, R.K. (1824b). On the genus Tortula, of the order Musci. Edinburgh J. Sci 1: 287-302.

Ireland, R.R. (1969). A taxonomic revision of the genus Plagiothecium for North America, north of Mexico. Publ. Bot. Nat. Mus. Nat. Sci. Can. 1: 1-118.

Iwatsuki, Z. (1972). Geographical isolation and speciation of bryophytes in some islands of Eastern Asia. J. Hattori Bot. Lab. 35: 126-141.

Iwatsuki, Z. \& Noguchi A. (1973). Index muscorum, japonicorum. J. Hattori Bot. Lab. 37: 299-418.

Iwatsuki, Z. \& Noguchi, A. (1979). Index muscorum, japonicorum. Supplement 1973-1978. J. Hattori Bot. Lab. 46: 235-255.

Iwatsuki, Z. \& Crosby, M.R. (1978). Lectotypification of the genus Isopterygium Mitt. J. Hattori Bot. Lab. 45: 389-394.

Jacobs, S.W.L. \& Pickard, J. (1981). 'Plants of New South Wales.' (Govt Printer: Sydney.)

Jaeger, A. \& Sauerbeck, F. (1876-9). 'Genera et Species Muscorum Systematice Disposita seu Adumbratio Florae Muscorum Totius Orbis Terrarum.' (Zollikofer: St. Gallen.) 2 vols.

Karczmarz, K. (1966). Taxonomic studies on the genus Acrocladium Mitt. Nova Hedwigia 11(1-4): 499-505.

Kindberg, N.C. (1901). Grundzüge einer Monographie über die Laubmoos-Familie Hypopterygiaceae. Hedwigia 40: 275-303.

Kindberg, N.C. (1902). Grundzuige einer Monographie der Laubmoos-Gattung Thamnium. Hedwigia 41: 203-268.

Koponen, A. (1981). Splachnobryaceae, a new moss family. Ann. Bot. Fen. 18(2): 123-132.

Koponen, A. (1982a). The family Splachnaceae in Australasia and the Pacific. $J$. Hattori Bot. Lab. 52: 87-92.

Koponen, A. (1982b). The generic classification of the Splachnaceae. Nova Hedwigia 71: 239-247.

Koponen, T. (1968). Generic revision of Mniaceae Mitt. (Bryophyta). Ann. Bot. Fenn. 5: 117-151.

Koponen, T. (1972). The east Asiatic species of Plagiomnium sect. Rostrata (Bryophyta). Acta Bot. Fenn. 97: 1-29.

Koponen, T. (1977a). Modern taxonomical methods and the classification of mosses. Bryophyt. Biblioth. 13: 443-481.

Koponen, T. (1977b). Miscellaneous notes on Mniaceae (Bryophyta) I. New combinations. Ann. Bot. Fenn. 14: 6.

Koponen, T. (1979a). A synopsis of Mniaceae (Bryophyta) I. South and Central American taxa. J. Hattori Bot. Lab. 46: 155-161.

Koponen, T. (1979b). On the taxonomy and phytogeography of Mnium Hedw. s. str. (Musci, Mniaceae). Abstr. Bot. 5 (Suppl. 3): 63-73.

Koponen, T. (1979c). A synopsis of Mniaceae (Bryophyta) II. Orthomnion. Ann. Bot. Fenn. 16: 35-58.

Koponen, T. (1979d). Miscellaneous notes on Mniaceae (Bryophyta). IV. Typification of Hedwig's species of Mniaceae. Ann. Bot. Fenn. 16: 79-89. 
Kopunen, T. (1980). A synopsis of Mniaceae (Bryophyta) III. Carl Müller's undescribed and dubious names. Ann. Bot. Fenn. 17: 61-64.

Koponen, T. (1981) A synopsis of Mniaceae (Bryophyta) VII. List of species and their distribution. Ann. Bot. Fenn. 18(2): 113-115.

Koponen, T. (1982). The family Mniaceae in Australasia and the Pacific. J. Hattori Bot. Lab. 52: 75-86.

Labillardière, J.J. (1806-7). 'Novae Hollandiae Plantarum Specimen.' (Huzard: Paris; repr. 1966 Cramer: Lehre). pp. 106-108.

Lane, D. (1978). A geographical guide to bryophyte floras of the world. Misc. Bryol. Lichénol. 8(1): 15-34.

Lawton, E. (1972). The genus Rhacomitrium in America and Japan. J. Hattori Bot. Lab. 35: 252-262.

Lewinsky, J. (1976). On the systematic position of Amphidium Schimp. Lindbergia 3: $227-231$.

Lewinsky, J. (1977). The genus Orthotrichum. Morphological studies and evolutionary remarks. J. Hattori Bot. Lab. 43: 31-61.

Lewinsky, J. (1978). The genus Orthotrichum Hedw. (Musci) in Africa south of the Tropic of Cancer. Bot. Tidsskr. 72(2-3): 61-85.

Lewinsky, J. (1980). Orthotrichum hurunii R. Brown ter. is Ptychomitrium australe (Hampe) Jaeg. Lindbergia 6(1): 19-21.

Lin, S-H. (1983). A taxonomic revision of Phyllogoniaceae (Bryopsida) Part I. $J$. Taiwan Mus. Assoc. 36(2): 37-86.

Lindberg, S.O. (1870a). Nya mossor. Ofvers. Förh. Kongl. Svenska Vetensk.-Akad. 12: $70-84$.

Lindberg, S.O. (1870b). Contributions to British bryology. J. Linn. Soc., Bot. 11: 460-468.

Lindberg, S.O. (1875). On a new moss from Tasmania. London J. Bot. 13: 167-168.

Lothian, N. (1955). Mosses in South Australia. S. Austral. Naturalist 30: 25.

McVean, D.N. (1969). Alpine vegetation of the central Snowy Mountains of New South Wales. J. Ecol. 57: 67-86.

Magill, R.E. (1982). On the circumspection of Pterobryaceae. Nova Hedwigia 71: 273-279.

Magill, R.E. \& Schelpe, E.A. (1979). The bryophytes of Southern Africa. An annotated checklist. Mem. Bot. Surv. South Africa 43: 1-39.

Malta, N. (1923-4). Studien über die Laubmoosgattung Zygodon Hook. et Tayl. (1-4) Latv. Augstsk. Rak. 5: 187-192; (5-9) 6: 273-295; (10) 9:111-153; (11-12) 10: 303-335.

Malta, N. (1926). Die Gattung Zygodon Hook. et Tayl. Eine monographische Studie. Acta Horti Bot. Univ. Latv. (Darbi) 1: 1-185.

Malta, N. (1933). A survey of the Australasian species of Ulota. Acta Horti Bot. Univ. Latv. (Raksti) 7: 1-24.

Manuel, M. G. (1974). A revised classification of the Leucodontaceae and a revision of the subfamily Alsioideae. Bryologist 77(4): 531-550.

Manuel, M.G. (1980). Miscellanea bryologia II - Classification of Rhizogonium Brid., Penzigiella hookeri Gangulee and some nomina nuda. Cryptog. Bryol. Lichénol. 1: 67-72.

Manuel, M.G. (1982). A brief review of the systematics of Leucodontaceae and Cryphaeaceae. Nova Hedwigia 71: 281-295.

Margadant, W.D. (1968). 'Early Bryological Literature. A Descriptive Bibliography of Selected Publications Treating Musci During the First Decades of the Nineteenth Century and Especially of the Years 1825, 1826 and 1827.' (Hunt Botanical Library: Pittsburgh.)

Margadant, W.D. (1972). Notes on the nomenclature of Musci. Lindbergia 1(3-4): 121-129.

Margadant, W.D., Miller, H.A. \& Matteri, C.M. (1972). Proposal for the conservation of the generic name Ptergophyllum Brid. Taxon 21: 536. 
Margadant, W.D. \& Terken, L. (1981). 'Index of Author Abbreviations in the Index Muscorum.' (Instituut voor Geschiedeins van de Biologie: Utrecht.)

Marginson, M.A. \& Murray-Smith, S. (1969). Further investigations in the Kent Group (Bass Strait Islands). Victorian Naturalist 86(5): 254-268.

Martin, W. (1946). Geographic range and internal distribution of the mosses indigenous to New Zealand. Trans. Roy. Soc. New Zealand 76(2): 162-184.

Martin, W. (1949). Distribution of the mosses indigenous to New Zealand Supplement I. Trans. Roy. Soc. New Zealand 77(3): 355-360.

Martin, W. (1951). Notes on the moss flora of New Zealand. Trans. Brit. Bryol. Soc. 1(5): 471-474.

Martin, W. (1957). G.O.K. Sainsbury, F.L.S. Bryologist 60: 363-367.

Matteri, C.M. (1968). Las especies de Philonotis (Bartramiaceae) del sur de Argentina. Revista Mus. Argent. Ci. Nat. Bernardino Rivadavia. 3(4): $185-234$.

Matteri, C.M. (1972). Las Hookeriaceae (Musci) Andino-Patagonicas. II. Mus. Argent. Ci. Nat. Bernardino Rivadavia. 4(2): 243-280.

Matteri, C.M. (1973a). Revision de las Hypopterygiaceae (Musci) austrosudamericas. Bol. Soc. Argent. Bot. 15: 229-250.

Matteri, C.M. (1973b). El genero 'Breutelia' (Bartramiaceae, Musci) en la region Andino-Patagonica. Revista Mus. Argent. Ci. Nat. Bernadino Rivadavia 4(4): 323-359.

Matteri, C.M. (1976). Proposal for typification of genus Hypopterygium Brid. Taxon 25: 189.

Matteri, C.M. (1981). Patagonian bryophytes 4 - Breutelia elongata (Hook. f. et Wils.) Mitt. and Myurella julacea (Schwaegr.) B.S.G. Lindbergia 7(1): 61-65.

Meijer, W. (1952). The genus Orthodontium. Acta Bot. Neerl. 1: 3-80.

Miller, H.A. (1971). Pterygophyllum Brid. is a good name. Taxon 20: 382-383.

Miller, N.G. \& Manuel, M.G. (1982). Trachyloma (Bryophytina, Pterobryaceae): a taxonomic monograph. J. Hattori Bot. Lab. 51: 273-322.

Mitten, W. (1856). A list of the musci and hepaticae collected in Victoria, Australia, by Dr. F. Mueller. Hooker's J. Bot. Kew Gard. Misc. 8: 257-266.

Mitten, W. (1860). Description of some new species of musci from New Zealand and other parts of the southern hemisphere, together with an enumeration of the species collected in Tasmania by William Archer Esq.; arranged upon the plan proposed in the 'Musci Indiae Orientalis'. J. Linn. Soc., Bot. 4: 64-100.

Mitten, W. (1882). Australian mosses, enumerated by William Mitten Esq. Trans. \& Proc. Roy. Soc. Victoria 19: 49-96.

Mohamed, M.A. Haji (1979). A taxonomic study of Bryum billardieri Schwaegr. and related species. J. Bryol. 10(4): 401-466.

Montagne, J.F.C. (1844). Plantes cellulaires. In Dumont d'Urville, J.S.C. 'Voyage au Pole Sud et dans l'Océanie sur les Corvettes l'Astrolabe et la Zélée, Exécuté par Ordre du Roi pendant les Années 1837-1840, sous le Commandement de M.J. Dumont d'Urville, Capitaine De Vaisseau.' (Gide and Cie: Paris.) Vol. 1, pp. 281-335.

Moore, C.J. \& Scott, G.A.M. (1979). The ecology of mosses on a sand-dune in Victoria, Australia. J. Bryol. 10: 291-311.

Mueller, D.M. (1973). The peristome of Fissidens limbatus Sullivant. Univ. Calif. Publ. Bot. 63: 1-34.

Mueller, F. von (1854). Second general report of the government botanist on the vegetation of the Colony. Victoria (Parliamentary Pap.) Votes and Proc. Legislative Council 1854-5 1: 17-18.

Mueller, F. von (1858). Annual Report of the Government Botanist and Director of the Botanic Garden. Victoria (Parliamentary Pap.) Votes and Proc. Legislative Council 1858-9 2: 12-13. 
Mueller, F. von (1864). 'Analytical Drawings of Australian Mosses.' (Govt Printer: Melbourne.)

Mueller, F. von (1880). 'Fragmenta Phytographiae Australiae.' (Govt Printer: Melbourne.) Vol: 11, Suppl. pp. 107-115.

Mueller, F. von (1882). Australian mosses, enumerated by William Mitten Esq. See Mitten (1882).

Müller, C. (1848-51). 'Synopsis Muscorum Frondosorum Omnium Hucusque Cognitorum.' (Foerstner: Berlin.) 2 parts.

Müller, C. (1851). Die, von Samuel Mossman im Jahre 1850, in Van Dieman's Land, Neuseeland und Neuholland gemachte Laubmoossammlung. Bot. Zeitung (Berlin) 9: 545-552, 561-567.

Müller, C. (1868). Beitrag zur ostaustralischen Moosflor. Linnaea 35: 613-626.

Müller, C. (1871). Musci Australici praesertim Brisbanici novi. Linnaea 37: 143-162.

Müller, C. (1874a). Musci polynesiaci praesertim Vitiani et Samoani Graeffeani. $J$. Mus. Godeffroy 3(6): 51-90.

Müller, C. (1874b). Novitates Bryothecae Mullerianae publicavit Carolus Muller Hal. 2. Musci Novo-Granatenses Wallisiani adjectis nonnullis aliis muscis novis andinis vel tropico-Americanis vel australasiacis. Linnaea 38: $572-620$.

Müller, C. (1883). Die auf der Expedition S.M.S. "Gazelle" von Dr. Naumann gesammelten Laubmoose. Bot. Jahrb. Syst. 5: 76-88.

Müller, C. (1887). Sphagnorum novorum descriptio. Flora 70: 403-422.

Müller, C. (1888). Musci cleistocarpici novi. Flora 71: 1-14.

Müller, C. (1889). Laubmoose (Musci Frondosi). In Naumann, F.C. (ed.) 'Die Forschungsreise S.M.S. Gazelle in den Jahren 1874 bis 1876 unter Kommando des Kapitan Zur Zee Freiherrn von Schleinitz.' (Admiralty Hydrographic Dept: Berlin.) Vol. IV, pp 1-64.

Müller, C. (1897a). Synopsis generis Harrisonia. Oesterr. Bot. Z. 47(11): 387-398; 47(12): 417-420.

Müller, C. (1897b). Triquetrella genus Muscorum novum conditum et descriptum. Oesterr. Bot. Z. 47 (12): 420-424.

Müller, C. (1897c). Symbolae ad Bryologiam Australiae I. Hedwigia 36: 331-365.

Müller, C. (1898). Symbolae ad Bryologiam Australiae II. Hedwigia 37: 76-171.

Müller, C. (1901). 'Genera Muscorum Frondosorum.' (Kummer: Leipzig.)

Müller, C. (1902). Symbolae ad Bryologiam Australiae III. Hedwigia 41: 119-134.

Müller, C. \& Brotherus, V.F. (1900). Ergebnisse einer Reise nach dem Pacific (H.

Schauinsland 1896/7). Musci Schauinslandiani. Ein Beitrag zur

Kenntnis der Moosflora der Pacifischen Inseln. Abh. Naturwiss. Vereine Bremen 16(3): 493-512.

Müller, C. \& Hampe, E. (1853). Musci frondosi Australasiae ab Dre. Ferd. Müller lecti. Linnaea 26: 489-505.

Noguchi, A. (1976). A taxonomic revision of the family Meteoriaceae in Asia. $J$. Hattori Bot. Lab. 41: 231-257.

Nowak, H. (1980). Mittythyridium in Ozeanien. Bryophytum Bibliotheca 20: 1-236.

Nyholm, E. (1965). 'Illustrated Moss Flora of Fennoscandia.' (Gleerup: Lund.) II. Fasc. 5.

Nyholm, E. (1971). Studies in the genus Atrichum P. Beauv. A short survey of the genus and the species. Lindbergia 1: 1-33.

Ochi, H. (1967). Notes on moss flora V. Hikobia 5(102): 14-38.

Ochi, H. (1968a). On the status of Bryum handelii Broth. (Musci). J. Jap. Bot. 43: 480-485.

Ochi, H. (1968b). A revised list of mosses of the family Bryaceae in Japan and the adjacent regions. J. Fac. Educ. Tottori Univ. Nat. Sci. 19(1): 24-40.

Ochi, H. (1969). Notes on moss flora VI. Hikobia 5(3-4): 153-171.

Ochi, H. (1970). A revision of the subfamily Bryoideae in Australia, Tasmania, New Zealand and the adjacent islands. J. Fac. Educ. Tottori Univ. Nat. Sci. 21(1): 7-67. 
Ochi, H. (1971). What is true Bryum truncorum? Bryologist 74: 503-506.

Ochi, H. (1972). Some problems of distributional patterns and speciation in the subfamily Bryoideae in the regions including Eurasia, Africa and Oceania. J. Hattori Bot. Lab. 35: 50-67.

Ochi, H. (1973). Supplement to the family Bryoideae (Musci) in Australia and New Zealand. Hikobia 6(3-4): 217-223.

Ochi, H. (1974). Some Bryaceous "Old World" mosses, also distributed in the New World. J. Fac. Educ. Tottori Univ. Nat. Sci. 25(1-2): 35-41.

Ochi, H. (1982). A phytogeographical consideration of Australasian Bryoideae in relation to those in other continents. J. Hattori Bot. Lab. 52: 65-74.

Paris, E.G. (1903-6). 'Index Bryologicus sive Enumeratio Muscorum ad Diem Ultimam Anni 1900 Cognitorum Adjunctis Synonymia Distributioneque Geographica Locupletissimis.' (Hermann: Paris.) Edn $2 ; 5$ vols.

Petrie, H.K. (1925). An ecological study of the flora of Mt. Wilson. Proc. Linn. Soc. New South Wales 50: 146-166.

Proskauer, J. (1958). On the peristome of Funaria hygrometrica. Amer. J. Bot. 45: $560-563$.

Ramsay, H.P. (1964). The chromosomes of Dawsonia. Bryologist 67(2): 153-162.

Ramsay, H.P. (1966a). Cytological Studies of Australian Mosses. Ph. D. Thesis, University of Sydney.

Ramsay, H.P. (1966b). Sex chromosomes in Macromitrium. Bryologist 69(3): 293-311.

Ramsay, H.P. (1967a). Intraspecific polyploidy in Hypopterygium rotulatum (Hedw.) Brid. Proc. Linn. Soc. New South Wales 91(3): 220-230.

Ramsay, H.P. (1967b). IOPB Chromosome Number reports. XIV. Taxon 16: $552-561$.

Ramsay, H.P. (1973). Unusual sporocytes in Dicnemoloma pallidum (Hook.) Wijk \& Marg. Bryologist 76: 178-182.

Ramsay, H.P. (1974). Cytological studies of Australian mosses. Austral. J. Bot. 22: 293-348.

Ramsay, H.P. (1977). Chromosome numbers of some mosses from Western Australia. J. Bryol. 9: 343-347.

Ramsay, H.P. (1979). Anisospory and sexual dimorphism in the Musci. In Clarke, G.C.S. and J.G. Duckett (eds), 'Bryophyte Systematics.' (Academic Press: London \& New York.) Vol. 14, pp. 281-316.

Ramsay, H.P. (1980). Contributions of Rev. W.W. Watts F.L.S. to Australian Botany. Taxon 29(4): 455-469.

Ramsay, H.P. (1984a). Phytogeography of the mosses of New South Wales. Telopea 2(5): $535-547$.

Ramsay, H.P. (1984b). The mosses of Lord Howe Island. Telopea 2(5): 549-558.

Ramsay, H.P. \& Briggs, B. (1979). The bryophyte and lichen collections at the National Herbarium of New South Wales. Taxon 28(4): 639-640.

Ramsay, H.P. \& Streimann, H. (1984). Mosses and their distribution in the Australian Capital Territory. Telopea 2(5): 559-574.

Ratkowsky, D.A. (1980). Some further synonyms of Bryoerythrophyllum jamesonii (Tayl.) Crum. Bryologist 83(3): 367.

Reader, F.M. (1898a). Contributions to the flora of Victoria. 5. Description of new species of moss. Victorian Naturalist 15: 31.

Reader, F.M. (1898b). Contributions to the flora of Victoria. 6. Descriptions of new mosses. Victorian Naturalist 15: 59.

Reed, C.F. \& Robinson, H.E. (1972). Index to Die natürlichen Pflanzenfamilien (Musci-Hepaticae). Editions 1 and 2. Contr. Reed Herb. Baltimore 21.

Reichardt, H.W. (1870). Fungi, hepaticae et musci frondosi. In Fenzl, E. (ed.) 'Reise der Osterreichischen Fregatte Novara um die Erde in den Jahren 1857-1859 unter den Befehlen des Commodore B. von WüllerstorfUrbair.' (K.K. Hofund Staatsdruckerei: Wien.) Vol. 1, pp. 133-196. 
Renauld, F. (1901). Nouvelle classification des Leucoloma. Rev. Bryol. 28(4-5): 66-70, 85-87.

Richards, P.W. (1963). Campylopus introflexus (Hedw.) Brid. and C. polytrichoides De Not. in the British Isles; a preliminary account. Trans. Brit. Bryol. Soc. 4(3): 404-417.

Robinson, H. (1970). A revision of the moss genus, Trichostompsis. Phytologia 20: 186-191.

Robinson, H. (1971). A revised classification for the order and families of mosses. Phytologia 21: 289-293.

Robinson, H. \& Norris, D.H. (1981). Stoneobryum, a new genus of Orthotrichaceae from South Africa and Southern Queensland. Bryologist 84(1): 95-99.

Rodway, L. (1914). 'Tasmanian Bryophyta.' (Roy. Soc. Tasmania: Hobart.) Vol. 1.

Rodway, L. (1915). Additions to the Tasmanian flora. Pap. \& Proc. Roy. Soc. Tasmania: 104-107.

Rodway, L. (1916). Additions to the Tasmanian flora. Pap. \& Proc. Roy. Soc. Tasmania: 44-47.

Roth, G. (1911). 'Die aussereuropäischen Laubmoose.' (Heinrich: Dresden.) Band I.

Roth, G. (1913). Nachtrag I. zu Band I der aussereuropäischen Laubmoose von 1910/11. Hedwigia 53: 81-98.

Roth, G. (1914). Nachtrag II zu Band I der aussereuropäischen Laubmoose von 1910/11. Hedwigia 54: 267-274.

Sainsbury, G.O.K. (1932). The study of Australian Mosses. Victorian Naturalist 48: 255-260; 49: 46-49, 77-80, 108-111.

Sainsbury, G.O.K. (1945). New and critical species of New Zealand mosses. Trans. Roy. Soc. New Zealand 75(2): 169-186.

Sainsbury, G.O.K. (1947). Additions to the mosses of Victoria. Victorian Naturalist 63(10): 222-223.

Sainsbury, G.O.K. (1948). Bryobartramiaceae, a new moss family. Bryologist 51: 9-13.

Sainsbury, G.O.K. (1953a). Two new species of Tasmanian mosses. Victorian Naturalist 70: 30-31.

Sainsbury, G.O.K. (1953b). Notes on Tasmanian mosses from Rodway's Herbarium. Pap. \& Proc. Roy. Soc. Tasmania 87: 83-91.

Sainsbury, G.O.K. (1955a). A handbook of the New Zealand Mosses. Bull. Roy. Soc. New Zealand 5: 1-490.

Sainsbury, G.O.K. (1955b). Notes on Tasmanian mosses from Rodway's Herbarium. 2-6. Pap. \& Proc. Roy. Soc. Tasmania 89: 1-11, 13-20, 21-35, 37-43, 45-53.

Sainsbury, G.O.K. (1956a). Notes on Tasmanian mosses from Rodway's Herbarium. 7-8. Pap. \& Proc. Roy. Soc. Tasmania 90: 35-39, 41-47.

Sainsbury, G.O.K. (1956b). A new species of Pottia from Central Australia. Rev. Bryol. Lichénol. 25: 237-238.

Salmon, E.S. (1899). On the genus Fissidens. Ann. Bot. 13: 103-130.

Salmon, E.S. (1900). Bryological Notes (8). Eccremidium exiguum (Hook. f. \& Wils.). Rev. Bryol. 27(6): 85-86.

Saito, K. (1975). A monograph of Japanese Pottiaceae (Musci). J. Hattori Bot. Lab. 39: 373-537.

Sarafis, V. (1971). A biological account of Polytrichum commune. New Zealand J. Bot. 9(4): 711-724.

Sayre, G., Bonner, C.E.B. \& Culberson, W.L. (1964). The authorities for the epithets of mosses, hepatics and lichens. Bryologist 67: 113-135.

Schelpe, E.A.C.L.E. (1970). A provisional check-list of the bryophyta of the Cape Peninsula. Contr. Bolus Herb. 2: 49-70.

Schofield, W.B. (1974). Bipolar disjunctive mosses in the Southern Hemisphere, with particular reference to New Zealand. J. Hattori Bot. Lab. 38: 13-32.

Schofield, W.B. \& Crum, H.A. (1972). Disjunctions in Bryophytes. Ann. Missouri Bot. Gard. 59(2): 174-202. 
Schultze-Motel, W. (1970a). Monographie der Laubmoosgattung Andreaea. I. Die costaten Arten. Willdenowia 6: 25-110.

Schultze-Motel, W. (1970b) Ephemeropsidaceae - ein neuer Name für eine Familie der Laubmoose. Taxon 19(2): 251-252.

Schwaegrichen, C.F. (1811-42). 'Species Muscorum ... Supplementum.' (Barth: Leipzig.) 7 vols.

Scott, G.A.M. (1971). A bibliography of New Zealand bryology. New Zealand J. Bot. 9: 750-771.

Scott, G.A.M. (1982). Bryofloristics in Australasia. Nova Hedwigia 71: 483-493.

Scott, G.A.M. \& Stone, I.G. (1976). 'The Mosses of Southern Australia (with illustrations by Celia Rosser).' (Academic Press: London, New York, San Francisco).

Scott, G.A.M. \& Stone, I.G. (1979). In defence of Dawsonia superba Grev. Lindbergia 5: 71-72.

Seppelt, R.D. (1977). Studies on the bryoflora of Macquarie Island. I. Introduction and checklist of species. Bryologist 80: 167-170.

Seppelt, R.D. (1980a). A study of Ditrichum difficile with special reference to Australian material. J. Bryol. 11(1): 33-42.

Seppelt, R.D. (1980b). Ditrichum brachycarpum, $D$. calcareum and $D$. subbrachycarpum in Australia. J. Bryol. 11(1): 43-48.

Seppelt, R.D. (1980c). The taxonomic status of Pseudodistichium Card. Ditrichaceae. Lindbergia 6: 126-128.

Seppelt, R.D. (1980d). What is Ditrichum falcifolium? Bryologist 83(4): 589-591.

Seppelt, R.D. (1982a). A monographic revision of the genus Ditrichum (Musci: Ditrichaceae) 1. Australian and New Zealand species. J. Hattori Bot. Lab. 51: 99-150.

Seppelt, R.D. (1982b). Ditrichum and other genera of Ditrichaceae in Australasia and the Pacific. J. Hattori Bot. Lab. 52: 107-112.

Seppelt, R.D. \& Stone, I.G. (1977) A comparison of vegetative features of Ditrichum cylindricarpum and Ditrichum punctulatum. J. Bryol. 9: 321-325.

Sim, T.R. (1926). The Bryophyta of South Africa. Trans. Roy. Soc. South Africa. 15: $1-475$.

Smith, A.J.E. (1976). 'The Moss Flora of Britain and Ireland.' (Cambridge Univ. Press: Cambridge, London, New York, Melbourne.)

Smith, A.J.E. \& Whitehouse, H.L.K. (1978). An account of British species of the Bryum bicolor complex, including $B$. dunense sp. nov. J. Bryol. 10: $29-47$.

Smith, G.G. (1962). The flora of granite rocks of the Porongurup Range, South Western Australia. J. \& Proc. Roy. Soc. Western Australia. 45: 18-23.

Smith, G.G. (1969). Sphagnum subsecundum in Western Australia. Western Austral. Naturalist 11(3): 56-59.

Smith, G.L. (1969). On Atrichopsis with notes on some austral Psilopilum species, Polytrichaceae. Bull. Torrey Bot. Club 96(1): 60-69.

Smith, G.L. (1971). Conspectus of the genera of Polytrichaceae. Mem. New York Bot. Gard. 21(3): 1-83.

Smith, G.L. (1972). Continental drift and the distribution of Polytrichaceae. $J$. Hattori Bot. Lab. 35: 41-49.

Smith, G.L. (1974). New developments in the taxonomy of Polytrichaceae. Epiphragm structure and spore morphology as generic characters. $J$. Hattori Bot. Lab. 38: 148-150.

Smith, G.L. (1975a). Neotropical Polytrichaceae I, II. Bryologist 78(2): 201-204.

Smith, G.L. (1975b). Neotropical Polytrichaceae III. Bryologist 78(4): 480-483.

Smith, G.L. (1976a). Neotropical Polytrichaceae IV. Bryologist 79(1): 93-95.

Smith, G.L. (1976b). Neotropical Polytrichaceae V. Bryologist 79(4): 221-222.

Smith, J.E. (1808). Characters of Hookeria, a new genus of mosses, with descriptions of ten species. Trans. Linn. Soc. Lond. 9: 272-282. 
Snider, J.A. (1975). A revision of the genus Archidium (Musci). J. Hattori Bot. Lab. 39: 105-201.

Sorsa, P. (1976). Spore wall structure in Mniaceae and some adjacent bryophytes. In Ferguson, I.K. and J. Muller (eds), 'The Evolutionary Significance of the Exine.' Linn. Soc. Symposium Series I (Academic Press: London.) pp. 211-299.

Stafleu, F. (1967). 'Taxonomic Literature. A Selective Guide to Botanical Publications with Dates, Commentaries and Types.' (Int. Bureau Pl. Taxon.: Utrecht.)

Stafleu, F.A. (ed.) (1978). 'International Code of Botanical Nomenclature.' (Int. Bureau Pl. Taxon.: Utrecht.)

Stearn, W.T. (1978). 'Botanical Latin.' (David Charles: Newton Abbott.)

Stirling, J. (1886). The Cryptogamia of the Australian Alps. Part 1. Proc. Roy. Soc. Victoria 22: 49-56.

Stone, I.G. (1961a). The gametophore and sporophyte of Mittenia plumula (Mitt.) Lindb. Austral. J. Bot. 9(2): 124-51.

Stone, I.G. (1961b). The highly refractive protonema of Mittenia plumula (Mitt.) Lindb. (Mitteniaceae). Proc. Roy. Soc. Victoria 74: 119-124.

Stone, I.G. (1971). The sporophyte of Tortula pagorum (Milde) De Not. Trans. Brit. Bryol. Soc. 6(2): 270-277.

Stone, I.G. (1973a). Two new species of Archidium from Victoria, Australia. Muelleria 2(4): 191-213.

Stone, I.G. (1973b). A new species of Brachydontium from Australia. J. Bryol. 7(3): 343-351.

Stone, I.G. (1976a). Alaticosta, a new subgenus of Acaulon in Australia. J. Bryol. 9(2): 213-227.

Stone, I.G. (1976b). A new species of Pleuridium from Australia. J. Bryol. 9: 229-238.

Stone, I.G. (1976c). A remarkable new moss from Queensland, Australia Viridivellus pulchellum new genus and species (new family Viridivelleraceae). J. Bryol. 9: 21-31.

Stone, I.G. (1977a). Some morphological and anatomical features of the monotypic genus Bryobartramia Sainsbury (Musci). Austral. J. Bot. 25: 141-157.

Stone, I.G. (1977b). Bruchia queenslandica, a new moss from tropical Queensland. J. Bryol. 9: 509-518.

Stone, I.G. (1978). Tortula oleaginosa: a new moss from Australia. J. Bryol. 10(2): 117-124.

Stone, I.G. (1979). Acaulon eremicola, a new moss from the Australian arid zone. J. Bryol. 10(4): 467-474.

Stone, I.G. (1980a). Phascopsis rubicundis a new genus and species of Pottiaceae (Musci) from Australia. J. Bryol. 11(1): 17-31.

Stone, I.G. (1980b). Weissia subg. Astomum in Australia and some comments on the affinity of Viridivellus. J. Bryol. 11(2): 231-243.

Stone, I.G. (1981a). Spore morphology and some other features of Goniomitrium Hook. et Wils. (Funariaceae). J. Bryol. 11(3): 491-500.

Stone, I.G. (1981b). Sciaromium elimatum Broth. et Watts and S. forsythii Broth. reduced to synonymy in Cratoneuropsis relaxa (Hook. et Wils.) Fleisch. in Broth. J. Bryol. 11(4): 843-844.

Stone, I.G. \& Catcheside, D.G. (1980). The peristome of Ischyrodon lepturus (Tayl.) Schelpe. J. Bryol. 11(1): 99-104.

Stone, I.G. \& Schelpe, E.A.C.L.E. (1973). Two new generic records of mosses for southern Africa J. S. African Bot. 39(2): 131-132.

Stone, I.G. \& Scott, G.A.M. (1973). Name changes in Australian mosses. J. Bryol. 7: 603-605.

Stone, I.G. \& Scott, G.A.M. (1981). Leptodontium paradoxum, a new moss from Australia. J. Bryol. 11(4): 701-708.

Streimann, H. \& Touw, A. (1981). New records for some Australian mosses. $J$. Hattori Bot. Lab. 49: 261-272. 
Sullivan, D. (1887). Mosses of Victoria, with brief notes. Victorian Naturalist 4: 106-110.

Syed, H. (1973). A taxonomic study of Bryum capillare Hedw. and related species. J. Bryol. 7(3): 265-326.

Takaki, N. (1967). A revision of Japanese Campylopus. J. Hattori Bot. Lab. 30: 213-248.

Tan, B.C. (1979). A new classification for the genus Physcomitrella B.S.G. J. Hattori Bot. Lab. 46: 327-336.

Thériot, I. (1922). Le problème du Leucobryum candidum. Bull. Soc. Bot. Genève, Sér. 2, 13: 217-225.

Touw, A. (1971). A taxonomic revision of the Hypnodendraceae (Musci.) Blumea 19(2): 211-354.

Touw, A. (1974). Some notes on taxonomic and floristic research on exotic mosses. J. Hattori Bot. Lab. 38: 123-128.

Touw, A. (1976). A taxonomic revision of Thuidium, Pelekium and Rauiella (Musci: Thuidiaceae) in Africa south of the Sahara. Lindbergia 3: 135-195.

Touw, A. (1978). The mosses reported from Borneo. J. Hattori Bot. Lab. 44: 147-176.

Venturi, G. De (1896). Notice sur quelques espèces d'Orthotrichum de l'Australie. Rev. Bryol. 23(4): 65-67.

Vitt, D.H. (1973). A revisionary study of the genus Macrocoma. Rev. Bryol. Lichénol. 39(2): 205-220.

Vitt, D.H. (1974). A key and synopsis of the mosses of Campbell Island, New Zealand. New Zealand J. Bot. 12: 185-210.

Vitt, D.H. (1976). A monograph of the genus Muelleriella Dusen. J. Hattori Bot. Lab. 40: 91-114.

Vitt, D.H. (1979). The moss flora of the Auckland Islands of New Zealand, with a consideration of habitats, origins and adaptations. Canad. J. Bot. 57(20): 2226-2263.

Vitt, D.H. (1980a). The genus Macrocoma I - Typification of names and taxonomy of species. Bryologist 83(4): 405-436.

Vitt, D.H. (1980b). The genus Macrocoma II - Geographical variation in the Macrocoma tenue - M. sullivani species complex. Bryologist 83(4): 437-450.

Vitt, D.H. (1980c). A comparative study of Andreaea acutifolia, $A$. mutabilis and A. rupestris. New Zealand J. Bot. 18(3): 367-377.

Vitt, D.H. (1982a). Populational variation and speciation in austral mosses. $J$. Hattori Bot. Lab. 52: 153-160.

Vitt, D.H. (1982b). The genera of Orthotrichaceae. Nova Hedwigia 71: 261-268.

Vitt, D.H. (1983). The New Zealand species of the pantropical genus Macromitrium (Orthotrichaceae: Musci): taxonomy, phylogeny and phytogeography. J. Hattori Bot. Lab. 54: 1-94.

Vitt, D.H. \& Crosby, M.R. (1972). Achrophyllum - a new name for a genus of mosses. Bryologist 75(2): 174-175.

Vitt, D.H. \& Ramsay, H.P. Monograph of Macromitrium in Australasia. MS.

Warnstorf, C. (1890). Beiträge zur Kenntniss exotischer Sphagna. Hedwigia 29: 179-258.

Warnstorf, C. (1891). Beiträge zur Kenntniss exotischer Sphagna. Hedwigia 30(1): 12-46. Figs. 1-5; 30(3): 127-180.

Warnstorf, C. (1893). Beitrăge zur Kenntniss exotischer Sphagna. Hedwigia 32(1): $1-17$

Warnstorf, C. (1897). Beiträge zur Kenntniss exotischer Sphagna. Hedwigia 36: $145-176$.

Warnstorf, C. (1898). Beiträge zur Kenntniss exotischer Sphagna. Bot. Zbl. 76(13): 417-423.

Warnstorf, C. (1900a). Weitere Beiträge zur Kenntniss der Torfmoose. Bot Zbl. 82: 7-14, 39-45, 65-76. 
Warnstorf, C. (1900b). Neue Beiträge zur Kenntniss europaischer und exotischer Sphagnumformen. Hedwigia 39: 100-110.

Warnstorf, C. (1907). Neue europaische und aussereuropaische Torfmoose. Hedwigia 47: 76-124.

Warnstorf, C. (1911). Sphagnales-Sphagnaceae (Sphagnologia universalis). In Engler, A. (ed.), 'Das Pflanzenreich.' (Engelmann: Leipzig.) Heft 51, pp. $1-546$.

Warnstorf, C. (1916a). Pottia-Studien als Vorarbeiten zu einer Monographie des genus "Pottia" Ehrh. sensu stricto. Hedwigia 58: 35-152.

Warnstorf, C. (1916b). Bryophyta nova europaea et exotica. Hedwigia 57: 62-131.

Watanabe, R. (1972). A revision of the family Thuidiaceae in Japan and adjacent areas. J. Hattori Bot. Lab. 36: 171-320.

Watson, E.V. (1955). 'British Mosses and Liverworts.' (Cambridge Univ. Press: Cambridge.)

Watts, W.W. (1899). Notes on some recently described species of N.S. Wales mosses. Proc. Linn. Soc. New South Wales 24: 374-376.

Watts, W.W. (1900). Notes on some new mosses from New South Wales. Proc. Linn. Soc. New South Wales 24: 632-633.

Watts, W.W. (1905). Some Melbourne mosses. Victorian Naturalist 21: 140-142.

Watts, W.W. (1906). Australian mosses. Some locality pictures. Bryologist 9: 34-36, 41.

Watts, W.W. (1912). The Sphagna of Australia and Tasmania. Proc. Linn. Soc. New South Wales 37(2): 383-389.

Watts, W.W. (1916). Some cryptogamic notes from the Botanic Gardens, Sydney. Proc. Linn. Soc. New South Wales 41: 377-386.

Watts, W.W. \& Whitelegge, T. (1902). Census Muscorum Australiensium. Proc. Linn. Soc. New South Wales 27: Supplement pp. 1-90.

Watts, W.W. \& Whitelegge, T. (1905). Census Muscorum Australiensium. Proc. Linn. Soc. New South Wales 30: Supplement pp. 91-163.

Weber, W.A. (1968). Preliminary list of mosses of the Australian Capital Territory. [duplicated list of $128 \mathrm{spp}$.]

Weber, W.A. (1972). The identity of Barbula pseudopilifera (Musci: Pottiaceae). Lindbergia 1: 214-216.

Weber, W.A. \& Simone, L.D. (1977). Tetraphis pellucida and T. geniculata scindulae as diagnostic features in bryophytes. Bryologist 80: 164-167.

Welch, W.H. (1970). Hookeriaceae species and distribution in Africa, Europe, Asia, Australia and Oceania. Proc. Indiana Acad. Sci. 79: 377-387.

Weymouth, W.A. (1894). Some additions to the moss flora of Tasmania. Pap. \& Proc. Roy. Soc. Tasmania 1893: 200-210.

Weymouth, W.A. (1896). Some additions to the moss flora of Tasmania. Pap. \& Proc. Roy. Soc. Tasmania 1895: 106-220.

Weymouth, W.A. (1903). Some additions to the bryological flora of Tasmania. Part III. Pap. \& Proc. Roy. Soc. Tasmania 1902: 115-132.

Weymouth, W.A. \& Rodway, L. (1922). Bryophyte notes. Pap. \& Proc. Roy. Soc. Tasmania 1921: 173-175.

Whitehouse, H.L.K. (1966). The occurrence of tubers in European mosses. Trans. Brit. Bryol. Soc. 5(1): 103-116.

Whitelegge, T. \& Brotherus, V.F. (1892). Notes and Exhibits [list of collection of 20 species from Lord Howe Island.] Proc. Linn. Soc. New South Wales 7: 277.

Wijk, R. Van Der (1957). Precursory studies in Malaysian Mosses. I. Revision of the genus Dawsonia R. Brown. Rev. Bryol. Lichénol. 26(1-2): 8-19.

Wijk, R. Van Der \& Margadant, W.D. (1958-62). New combinations in mosses 1-8. Taxon 7: 287-290; 8: 70-75, 106; 9: 50-52, 189-191; 10: 24-26; 11 : 221-223.

Wijk, R. Van Der, Margadant, W.D. \& Florschutz, P.A. (1959-69). 'Index Muscorum.' (Int. Bureau P1. Taxon.: Utrecht.) 5 vols. 
Wild, C.J. (1888a). Notes on some Queensland mosses. Proc. Roy. Soc. Queensland 5: 116-119.

Wild, C.J. (1888b). Bryological notes. Proc. Roy. Soc. Queensland 5: 148-150.

Wild, C.J. (1889a). Bryological notes. Proc. Roy. Soc. Queensland 6: 76-79.

Wild, C.J. (1889b). Bryological notes. Proc. Roy. Soc. Queensland 6: 104-105.

Willis, J.H. (1952). Systematic notes on Victorian mosses - 1. Victorian Naturalist 69: 15-18.

Willis, J.H. (1953a). Nemataceae, a moss family new to Australia. Nature 172: 127-128.

Willis, J.H. (1953b). The myth of Macromitrium in Western Australia (a moss note). Victorian Naturalist 69: 159-160.

Willis, J.H. (1953c). Systematic notes on Victorian mosses - 2. Victorian Naturalist 70: 55-57.

Willis, J.H. (1954a). Systematic notes on Victorian mosses - 3. Victorian Naturalist 70: 169-172.

Willis, J.H. (1954b). Mosses new to Western Australia. Victorian Naturalist 71: 8-12.

Willis, J.H. (1955a). Systematic notes on Victorian mosses - 4. Victorian Naturalist 71: $157-163$.

Willis, J.H. (1955b). Some further notes on Sphagnum. Victorian Naturalist 71: 189-190.

Willis, J.H. (1955c). Systematic notes on Victorian mosses - 5. Victorian Naturalist 72: 5-11.

Willis, J.H. (1955d). New and interesting moss records for Australia. Victorian Naturalist 72: 73-78.

Willis, J.H. (1955e). The present position of muscology in Victoria. (A Centennial Review). Muelleria 1(1): 55-59.

Willis, J.H. (1957a). Systematic notes on Victorian mosses-6. Victorian Naturalist 74: 23-25.

Willis, J.H. (1957b). New records on mosses for Australian States (Victoria, Tasmania and the Northern Territory). Victorian Naturalist 74: 101-105.

Willis, J.H. (1958). Additional notes on Northern Territory mosses. Victorian Naturalist 74: 189.

Willis, J.H. (1972). Checklist of mosses in far northwestern Victoria. Sunraysia Naturalists Research Trust Annual Rep. 9: 15-16.

Wilson, W. (1846a). Remarks on some rare mosses of the Southern Hemisphere. London J. Bot. 5: 142-144.

Wilson, W. (1846b). Remarks on the new species of Musci from Quito and Swan River, indicated by Dr. Taylor in the London Journal of Botany, Vol. V. p. 41. London J. Bot. 5: 447-455.

Wilson, W. (1854). Musci. In Hooker, J.D., 'The Botany of the Antarctic Voyage... (Reeve: London.) Vol. II [Flora Novae-Zelandiae], Part 2, pp. $57-125$.

Wilson, W. (1859). Musci. In Hooker, J.D., 'The Botany of the Antarctic Voyage...' (Reeve: London.) Vol. III [Flora Tasmaniae], Part 2, pp. 160-221.

Wilson, W. \& Hooker, J.D. (1845). Musci. In Hooker, J.D., 'The Botany of the Antarctic Voyage ...' (Reeve: London.) Vol. I [Flora Antarctica], Part 1, pp. $117-143$.

Wilson, W. \& Hooker, J.D. (1847). Musci. In Hooker, J.D., 'The Botany of the Antarctic Voyage ...' (Reeve: London.) Vol. I [Flora Antarctica], Part 2, pp. 395-423.

Worley, I.A. \& Iwatzuki, Z. (1970). A checklist of mosses of Alaska. Bryologist 73(1): 59-71.

Zander, R.H. (1972). Revision of the genus Leptodontium in the New World. Bryologist 75(3): 213-280. 
Zander, R.H. (1977). The tribe Pleuroweisieae (Pottiaceae, Musci) in middle America. Bryologist 80(2): 233-269.

Zander, R.H. (1978a). New combinations in Didymodon (Musci) and a key to the taxa in North America, north of Mexico. Phytologia 41(1): 11-32.

Zander, R.H. (1978b). A synopsis of Bryoerythrophyllum and Morinia (Pottiaceae) in the New World. Bryologist 81(4): 539-560.

Zander, R.H. (1979). Notes on Barbula and Pseudocrossidium (Bryopsida) in North America and an annotated key to taxa. Phytologia 44(4): 177-214.

Zander, R.H. (1979). Techniques for study of Pottiaceae. Taxon 28(5/6): 634-644.

Zanten, B.O. van (1973). A taxonomic revision of the genus Dawsonia R. Brown. Lindbergia 2:1-48.

Zanten, B.O. van (1977). New combinations in the genus Dawsonia R. Brown. Lindbergia 4(1-2): 133 .

Zanten, B.O. van (1978). Experimental studies on trans-oceanic long-range dispersal of moss spores in the Southern Hemisphere. Bryophytorum Biblioth. 13: 715-733.

Zanten, B.O. van \& Pócs, T. (1982). Distribution and dispersal of Bryophytes. Advances in Bryology 1: 479-562.

Unpublished and incomplete documents held in the library at the National Herbarium of New South Wales, Sydney.

Watts, W.W. (1896-1900). New South Wales mosses. [List of specimens with names supplied by experts, numbers 1-10,110 with list of 374 Tasmanian mosses, largely specimens of Weymouth.]

Watts, W.W. (1911-1916). New South Wales mosses [numbers 10,011 on].

Watts, W.W. (n.d.). Queensland mosses and hepatics [numbers 226-681].

Watts, W.W. (n.d.). Lord Howe Island mosses [numbers 1-538].

Watts, W.W. (n.d.). New Hebrides mosses [815 listings].

Watts, W.W. (n.d.). Unpublished census of N.S.W. mosses. [Revision of Census Muscorum Australiensum with pleurocarpi. This was incorporated into an unpublished census of N.S.W. mosses by Burges 1952.] 
INDEX

Page

498 Acanthocladium

468 Acaulon

490 Achrophyllum

494 Acrocladium

468 Aloina

494 AMBLYSTEGIACEAE (73)*

494 Amblystegium

483 Amphidium

492 Anacamptodon

460 Andreaea

460 ANDREAEACEAE (2)

493 Anomodon

463. Archidium

491 Astomum

499 Atrichum

480 AULACOMNIACEAE (34)

480 Aulacomnium

482 Aulacopilum

488 Barbella

468 Barbula

481 Bartramia

48I BARTRAMIACEAE (37)

481 Bartramidula

486 Bescherellia

464 Blindia

474 Brachymenium

495 BRACHYTHECIACEAE (74)

495 Brachythecium

480 Braithwaitea

481 Breutelia

464 Bruchia

474 BRYACEAE (24)

472 Bryobartramia

472 BRYOBARTRAMIACEAE (14A)

469 Bryoerythrophyllum

475 Bryum

499. Buxbaumia

499 BUXBAUMIACEAE (81)

494 Calliergon

494 Calliergonella

479 CALOMNIACEAE (31)

479 Calomnion

467 CALYMPERACEAE (12)

467 Calymperes

467 Calymperopsis

469 Calyptopogon

489 Calyptothecium

491 Calyptrochaeta

489 Camptochaete

494 Campylium

464 Campylopus

488 Catagonium

499 Catharinea

462 Ceratodon

462 Cheilothela

462 Chrysoblastella

481 Conostomum

494 Cratoneuropsis

469 Crossidium

486 Cryphaea

486 CR YPHAEACEAE (48)

498 Ctenidium

492 Cyathophorum

491 Cyclodictyon

486 Cyptodon

486 CYRTOPODACEAE (50)

486 Cyrtopus
Page

500 Dawsonia

500 DAWSONIACEAE (84)

469 Desmatodon

465 Dichodontium

465 Dicnemoloma

466 DICNEMONACEAE (9)

464 DICRANACEAE (8)

465 Dicranella

465 Dicranoloma

466 Dicranum

469 Didymodon

462 Distichium

491 Distichopyllum

462 DITRICHACEAE (5)

463 Ditrichum

494 Drepanocladus

463 Eccremidium

490 ECHINODIACEAE (62)

490 Echinodium

498 Ectropothecium

467 Encalypta

467 ENCAL YPTACEAE (13)

487 Endotrichella

496 Entodon

496 ENTODONTACEAE (75)

473 EPHEMERACEAE (18)

473 Ephemerum

491 Eriopus

482 ERPODIACEAE (40)

466 Eucamptodon

487 Euptychium

495 Eurhynchium

492 Fabronia

492 FABRONIACEAE (70)

461 Fissidens

461 FISSIDENTACEAE (3)

486 Forsstroemia

473 Funaria

473 FUNARIACEAE (19)

473 GIGASPERMACEAE (16)

473 Gigaspermum

487 Glyphothecium

479 Goniobryum

473 Goniomitrium

472 Grimmia

472 GRIMMIACEAE (15)

469 Gymnostomum

487 Hampeella

493 Haplohymenium

486 Hedwigia

486 HEDWIGIACEAE (47)

486 Hedwigidium

492 Helicodontiadelphus

466 Holomitrium

490 HOOKERIACEAE (65)

479 Hymenodon

469 Hymenostomum

498 HYPNACEAE (78)

480 HYPNODENDRACEAE (33)

480 Hypnodendron

498 Hypnum

492 HYPOPTERYGIACEAE (68)

492 Hypopterygium

492 Ischyrodon

499 Isopterygium 
Page

489 LEMBOPHYLLACEAE (61)

490 Lembophyllum

477 Leptobryum

495 Leptodictyum

489 Leptodon

469 Leptodontium

478 LEPTOSTOMATACEAE (25)

478 Leptostomum

479 Leptotheca

493 LESKEACEAE (71)

466 LEUCOBR YACEAE (11)

466 Leucobryum

466 Leucoloma

492 Lopidium

483 Macrocoma

483 Macromitrium

481 Meesia

481 MEESIACEAE (35)

496 Meiothecium

479 Mesochaete

488 METEORIACEAE (58)

488 Meteorium

463 Micromitrium

477 Mielichhoferia

479 Mittenia

479 MITTENIACEAE (30)

478 MNIACEAE (26)

478 Mniobryum

478 Mnium

487 Muellerobryum

463 Nanomitrium

489 Neckera

489 NECKERACEAE (60)

477 Orthodontium

488 Orthorrhynchium

483 ORTHOTRICHACEAE (42)

485 Orthotrichum

496 Oxyrnynchium

488 Papillaria

482 Philonotis

488 PHYLLOGONIACEAE (59)

474 Physcomitrium

474 Physcomittrella

488 Pilotrichella

478 Plagiomnium

496 PLAGIOTHECIACEAE (76)

496 Plagiothecium

495 Platyhypnidium

463 Pleuridium

499 Pogonatum

478 Pohlia

499 POLYTRICHACEAE (83)

500 Polytrichadelphus

500 Polytrichastrum

500 Polytrichum

489 Porotrichum

470 Pottia

468 POTTIACEAE (14)

493 Pseudoleskea

493 Pseudoleskeopsis

500 Psilopilum

487 PTEROBRYACEAE (57)

487 Pterobryella

487 Pterobryidium
Page

487 Pterobryon

470 Pterygoneurum

490 Pterygophyllum

482 PTYCHOMITRIACEAE (41)

482 Ptychomitrium

487 PTYCHOMNIACEAE (51)

487 Ptychomnion

497 Pungentella

479 Pyrrhobryum

472 Racomitrium

485 RACOPILACEAE (44)

485 Racopilum

486 Rhacocarpus

497 Rhaphidorrhynchium

497 Rhaphidostegium

479 RHIZOGONIACEAE (32)

480 Rhizogonium

478 Rhodobryum

496 Rhynchostegiella

496 Rhynchostegium

494 Saniona

491 Sauloma

472 Schistidium

485 Schlotheimia

494 Sciaromium

465 Sclerodontium

464 SELIGERIACEAE (7)

496 SEMATOPHYLLACEAE (77)

497 Sematophyllum

460 SPHAGNACEAE (1)

460 Sphagnum

482 Spiridens

482 SPIRIDENTACEAE (38)

474 SPLACHNACEAE (21)

474 Splachnobryum

474 Splachnum

463 Sporledera

467 Syrrhopodon

497 Taxithelium

474 Tayloria

470 Tetrapterum

489 Thamnium

489 Thamnobryum

493 THUIDIACEAE (72)

493 Thuidiopsis

493 Thuidium

470 Tortella

470 Tortula

471 Trachycarpidium

487 Trachyloma

466 Trematodon

497 Trichosteleum

471 Trichostomum

471 Tridontium

471 Triquetrella

499 Vesicularia

497 Warburgiella

494 Warnstorfia

471 Weissia

488 Weymouthia

498 Wijkia

485 Zygodon

* Family number according to Crosby \& Magill 1981. 